\title{
FVC2000: Fingerprint Verification Competition
}

\author{
D. Maio ${ }^{1}$, D. Maltoni ${ }^{1}$, R. Cappelli ${ }^{1}$, J. L. Wayman ${ }^{2}$, A. K. Jain ${ }^{3}$ \\ ${ }^{1}$ Biometric System Lab (BIOLAB) - DEIS, \\ University of Bologna, via Sacchi 3, 47023 Cesena - ITALY. \\ E-mail: $\{$ maio, maltoni, cappelli \}@csr.unibo.it; URL: http://www.csr.unibo.it/research/biolab \\ ${ }^{2}$ U.S. National Biometric Test Center, College of Engineering, \\ San Jose State University, San Jose, CA 95192 \\ E-mail: jlwayman@aol.com; URL: http://www.engr.sjsu.edu/biometrics \\ ${ }^{3}$ Pattern Recognition and Image Processing Laboratory, \\ Michigan State University, East Lansing, MI 48824, USA. \\ E-mail: jain@cps.msu.edu; URL: http://www.cse.msu.edu/prip
}

\begin{abstract}
Reliable and accurate fingerprint recognition is a challenging pattern recognition problem, requiring algorithms robust in many contexts. This competition attempts to establish a first common benchmark, allowing companies and academic institutions to unambiguously compare performance and track improvement in their fingerprint recognition algorithms. Four databases have been created using different "state-of the-art" sensor technologies and 11 algorithms have been extensively tested on them. We believe that the FVC2000 databases and results will be useful both to the participants and, more generally, to all practitioners in the field, as a benchmark for improving methods and for enabling unbiased evaluation of algorithms.
\end{abstract}

Index Terms - Fingerprint recognition, Performance evaluation, Biometric systems.

\section{INTRODUCTION}

In the last decade, interest in fingerprint-based biometric systems has grown significantly [1]. Activity on this topic increased in both academia and industry as several research groups and companies developed new algorithms and techniques for fingerprint recognition and as many new electronic fingerprint acquisition sensors were launched into the marketplace.

Nevertheless, to date only a few benchmarks have been available for comparing developments in this area. Developers usually perform internal tests over self-collected databases. In practice, the only largely diffused datasets are the (U.S.) National Institute of Standards and Technology (NIST) CR-ROMs [2] [3] containing thousands of images scanned from paper cards where fingerprints were impressed by rolling "nail to nail" inked fingers. Since these images significantly differ from those acquired electronically, they are not well-suited for testing "on-line" fingerprint systems. 
NIST recently released a database of live-image sequences [4]. Although suitable for studying plastic distortion affecting the on-line acquisition process, it contains 100 live-sequences from only 10 individuals, a dataset too small to allow recognition accuracy to be measured with high confidence.

The lack of standards has unavoidably lead to the dissemination of confusing, incomparable and irreproducible results, sometimes embedded in research papers and sometimes enriching the commercial claims of marketing brochures.

The aim of this initiative is to take the first steps toward the establishment of a common basis, both for academia and industry, to better understand the state-of-the-art and what can be expected from this technology in the future. We decided to "dress" this effort as an international open competition to boost interest and give our results larger visibility. $15^{\text {th }}$ ICPR 2000 was ideal for this purpose. Starting in late spring 1999, when the FVC2000 web site (http://bias.csr.unibo.it/fvc2000/) was set up, we broadly publicized this event, inviting all companies and research groups we were aware of to take part.

From the beginning, we premised that the competition was not an official performance certification of the participant biometric systems, as:

- the databases used in this contest have not been acquired in a real environment and according to a formal protocol [5] [6] [7] [8] (also refer to [11] for an example of performance evaluation on real applications).

- only parts of the participants' software are evaluated by using images from sensors not native to each system. In fact, fingerprint-based biometric systems often implement proprietary solutions to improve robustness and accuracy (e.g. quality control modules to reject poor quality fingerprints, visual feedback to help the user in optimally positioning his/her finger, using multiple fingerprint instances to build more reliable templates, etc.) and these contributions are here discounted.

According to the definition reported in [6] [7], FVC2000 should be conceived as a technology evaluation (with some analogies with the FERET contest organized by P. J. Philips on face recognition [9]). In fact, strictly citing [8]:

"The goal of a technology evaluation is to compare competing algorithms from a single technology. Testing of all algorithms is done on a standardized database collected by a "universal" sensor. Nonetheless, performance against this database will depend upon both the environment and the population in which it was collected. Consequently, the "three bears" rule might be applied, attempting to create a database that is neither too difficult nor too easy for the algorithms to be tested. Although 
sample or example data may be distributed for developmental or tuning purposes prior to the test, the actual testing must be done on data which has not been previously seen by algorithm developers. Testing is done using "off-line" processing of the data. Because the database is fixed, results of technology tests are repeatable."

In FVC2000, the "universal" sensor is actually a collection of four different sensors to better cover the recent advances in fingerprint sensing techniques. In fact, databases 1 and 2 were collected by using two small-size and low-cost sensors (optical and capacitive, respectively). Database 3 was collected by using a higher quality (large area) optical sensor. Finally, images in database 4 were synthetically generated by using the approach described in [10]. Each of the four databases contained 880 fingerprints from 110 different fingers, collected using the "three bears rule" (not too easy, not too hard), as estimated prior to the test from our collective subjective experiences with fingerprint recognition algorithms. Each database was split into a sequestered "test" set of 800 images (set A) and an open "training" set of 80 images made available to participants for algorithm tuning (set B).

In March 2000, after several months of active promotion, we had 25 volunteering participants (about 50\% from academia and 50\% from industry), far more than our initial expectation (so we had a lot of work to do!). By the end of April, the training sets were released to the participants.. After the submission deadline (June 2000) for the executable computer programs, the number of participants decreased to 11 (most of the initially registered companies withdrew). Some of the withdrawals were undoubtedly due to the lack of time necessary to make algorithms "compatible" with FVC2000 images, but most were probably caused by the misalignments that some participants found between the performance measured on the FVC2000 training sets and their internal test sets; whilst this is not necessarily a problem for an academic research group, it could play negatively for a company releasing accuracy numbers significantly different from those measured at FVC2000. Perhaps, we did not stress strongly enough in our "Call for Participation" that FVC2000 is a test of relative technology performance, not intended to predict performance in a real environment. In any case, the 11 algorithms (see Table I) received were more than anticipated, so we started working on the submitted executables to complete their evaluation by August 2000.

Section II of this report summarizes the FVC2000 submission rules, while Section III describes the four databases used. In Section IV we present the criteria and the procedures used for performance evaluation. Section V reports the overall performance of the participant algorithms on each database and concludes with a comparison of the average results (more detailed results are included in Appendix A). Finally, in Section VI, we draw some concluding remarks and discuss how we intend to continue supporting this initiative in the future. 


\begin{tabular}{|c|l|c|}
\hline \multicolumn{1}{|c|}{ ID } & \multicolumn{1}{|c|}{ Organization } & Type \\
\hline CETP & CEFET-PR / Antheus Technologia Ltda (Brasil) & Academic \\
\hline CSPN & Centre for Signal Processing, Nanyang Technological University (Singapore) & Academic \\
\hline CWAI & $\begin{array}{l}\text { Centre for Wavelets, Approximation and Information Processing, Department of } \\
\text { Mathematics, National University of Singapore (Singapore) }\end{array}$ & Academic \\
\hline DITI & Ditto Information \& Technology Inc. (Korea) & Company \\
\hline FPIN & FingerPin AG (Switzerland) & Company \\
\hline KRDL & Kent Ridge Digital Labs (Singapore) & Academic \\
\hline NSMI & Natural Sciences and Mathematics, Institute of Informatics (Macedonia) & Academic \\
\hline SAG1 & SAGEM SA (France) & Company \\
\hline SAG2 & SAGEM SA (France) & Company \\
\hline UINH & Inha University (Korea) & Academic \\
\hline UTWE & University of Twente, Electical Engineering (Netherlands) & Academic \\
\hline
\end{tabular}

Table I: Participant algorithms list: a four digit ID has been assigned to each algorithm. 7 algorithms are from academic groups and 4 algorithms are from companies (Sagem SA submitted 2 different algorithms).

\section{ALGORITHM SUBMISSION POLICY}

Each participant was required to submit two executables in the form of "win32 console application". The submission of UNIX executables was also possible, although not encouraged . Ten participants submitted Windows applications and just one participant (FPIN) submitted executables for Linux O.S.

According to the given specification, the executables take the input from command-line arguments and append the output to a text file. The input includes a database-specific configuration file; in fact, participants were allowed to submit 4 distinct configuration files CF1.cfg, CF2.cfg, CF3.cfg and CF4.cfg (one for each database) in order to adjust the algorithm's internal parameters according to each specific database. Configuration files are text files or binary files and their I/O is responsibility of the participant's code. Configuration files can also contain pre-computed data to save time during enrollment and matching.

- The first executable (ENROLL_XXXX) enrolls a fingerprint image and produces a template file; the command-line syntax is:

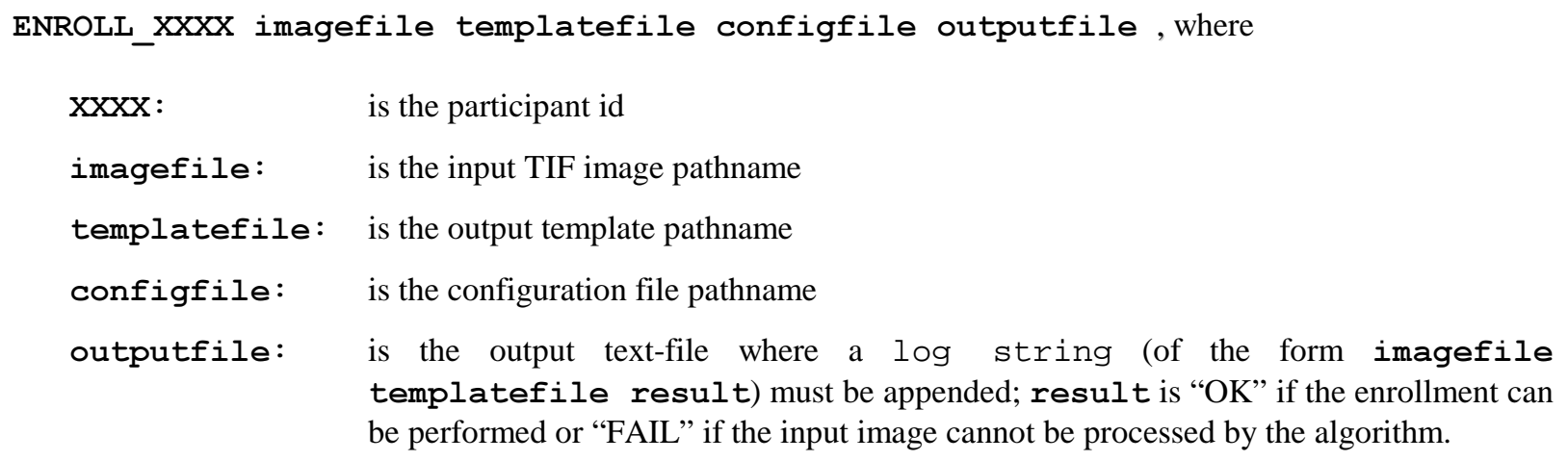


- The second executable (MATCH_XXXX) matches a fingerprint image against a fingerprint template and produces a similarity score; the command-line syntax is:

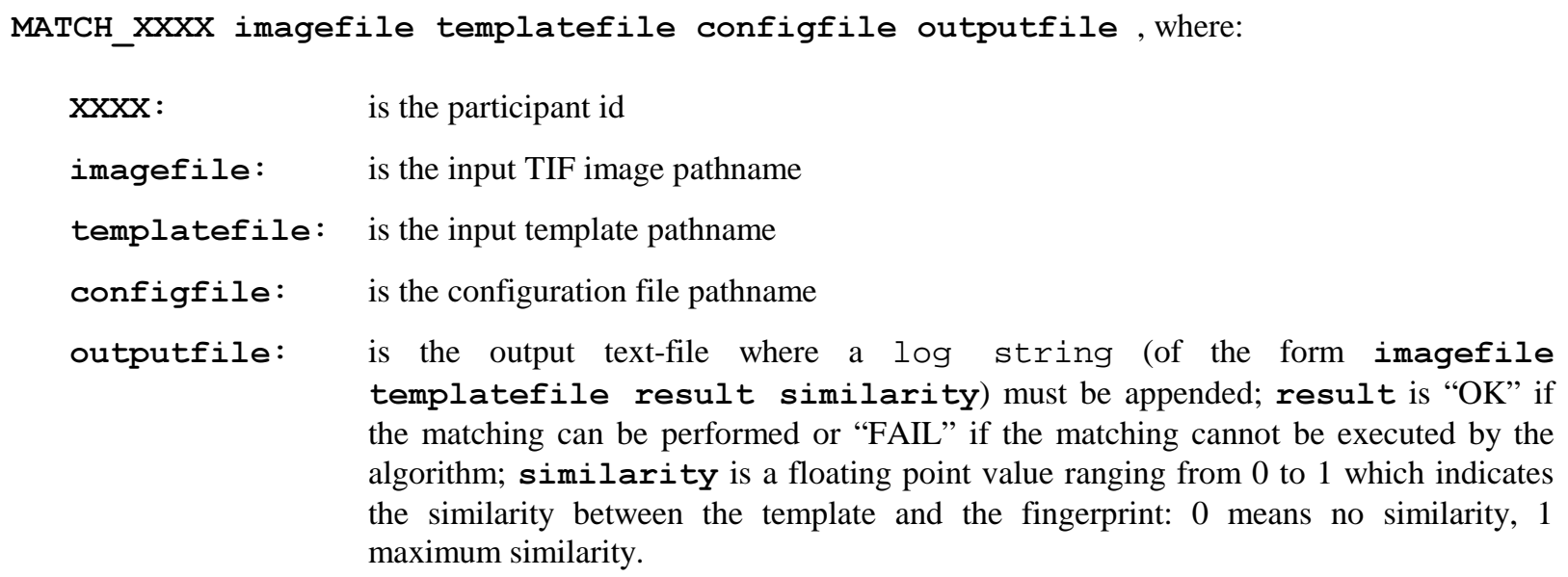
the matching can be performed or "FAIL" if the matching cannot be executed by the algorithm; similarity is a floating point value ranging from 0 to 1 which indicates the similarity between the template and the fingerprint: 0 means no similarity, 1 maximum similarity.

Two C-language skeletons for ENROLL_XXXX and MATCH_XXXX were made available on-line to reduce the participants implementation efforts. These source files perform all the necessary I/O (including TIF image loading).

We also premised that, for practical testing reasons, we should limit the maximum response time of the algorithms: max. 15 seconds for each enrollment, max. 5 seconds for each matching. The test was executed on Pentium III - 450 MHz machines (under Windows NT 4.0 and Linux RedHat 6.1).

Once all the executables were submitted, feedback was sent to the participants by providing them the results of their algorithms over the training set B (the same data set they had previously been given) to allow them to verify that neither run-time problems nor hardware-dependent misbehaviors were occurring on our side. 


\section{DATABASES}

Four different databases (hereinafter DB1, DB2, DB3 and DB4) were collected by using the following sensors/technologies:

DB1: low-cost optical sensor "Secure Desktop Scanner" by KeyTronic"

DB2: low-cost optical capacitive sensor “TouchChip" by ST Microelectronics*

DB3: optical sensor “DF-90" by Identicator Technology"

DB4: synthetic generation based on an evolution of the method proposed in [10].

Each database is 110 fingers wide (w) and 8 impressions per finger deep (d) (880 fingerprints in all); fingers from 101 to 110 (set B) have been made available to the participants to allow parameter tuning before the submission of the algorithms; the benchmark is then constituted by fingers numbered from 1 to 100 (set A).

Table II summarizes the global features of the four databases and Figure 1 shows a sample image from each of them.

\begin{tabular}{|l|c|c|c|c|c|}
\cline { 2 - 6 } \multicolumn{1}{c|}{} & Sensor Type & Image Size & Set A (w $\times \mathbf{d})$ & Set B (w $\times \mathbf{d})$ & Resolution \\
\hline DB1 & Low-cost Optical Sensor & $300 \times 300$ & $100 \times 8$ & $10 \times 8$ & $500 \mathrm{dpi}$ \\
\hline DB2 & Low-cost Capacitive Sensor & $256 \times 364$ & $100 \times 8$ & $10 \times 8$ & $500 \mathrm{dpi}$ \\
\hline DB3 & Optical Sensor & $448 \times 478$ & $100 \times 8$ & $10 \times 8$ & $500 \mathrm{dpi}$ \\
\hline DB4 & Synthetic Generator & $240 \times 320$ & $100 \times 8$ & $10 \times 8$ & About 500 dpi \\
\hline
\end{tabular}

Table II. The four FVC2000 databases.

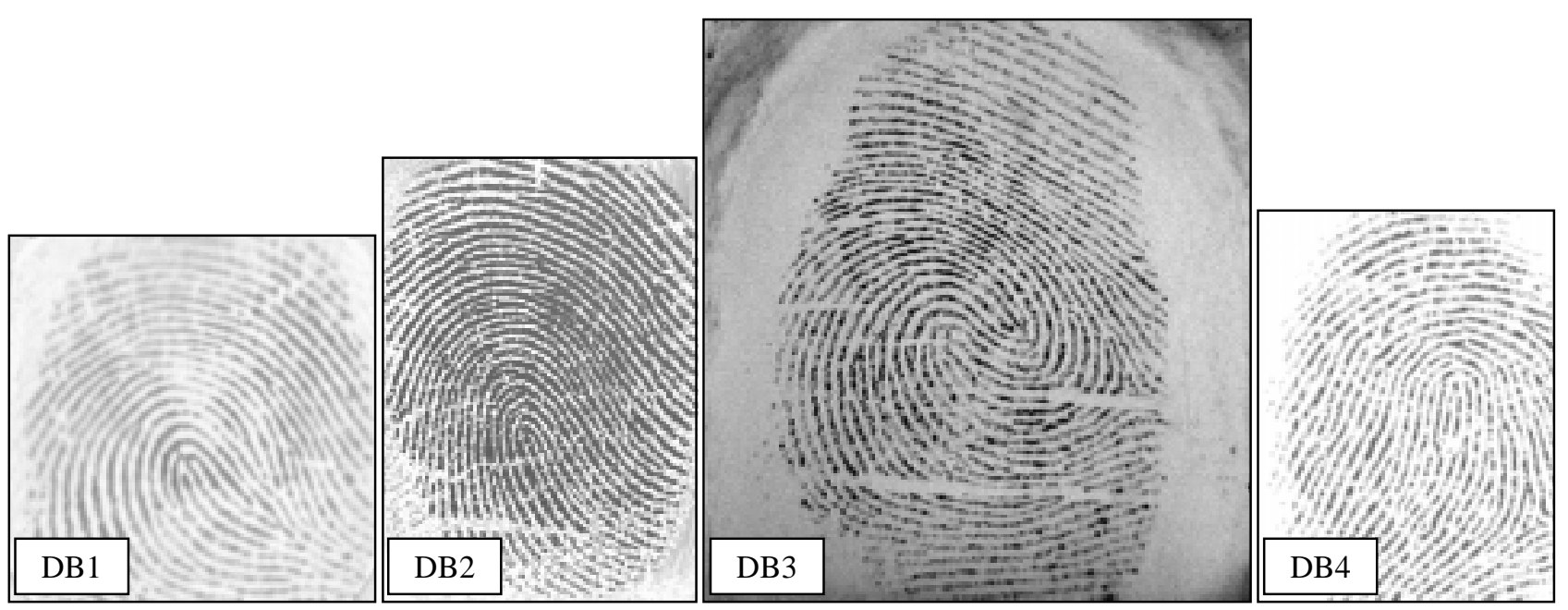

Figure 1. Sample images taken from DB1, DB2, DB3 and DB4. In order to show the different image size of each database, the four images are reported at the same scale factor.

\footnotetext{
"Some equipment are here identified in order to clearly specify the features of the databases. In no case such identification imply recommendation or endorsement by the authors, nor does it imply that these devices are necessarily the best available for the purpose.
} 
It is worth emphasizing that the choice of providing more than one database is not aimed at comparing different acquisition technologies and devices; the results obtained by the algorithms on the different databases should not be conceived as a quality measure of the corresponding sensors, since the acquisition conditions and the volunteer crew of each database are different.

More in detail, DB1 and DB2 have the following features:

- the fingerprints are mainly from 20 to 30 year-old students (about 50\% male).

- up to four fingers were collected for each volunteer (forefinger and middle finger of both the hands).

- the images were taken from untrained people in two different sessions and no efforts were made to assure a minimum acquisition quality.

- all the images from the same individual were acquired by interleaving the acquisition of the different fingers (e.g., first sample of left forefinger, first sample of right forefinger, first sample of left middle, first sample of right middle, second sample of the left forefinger, ...).

- the presence of the fingerprint cores and deltas is not guaranteed since no attention was paid on checking the correct finger centering.

- the sensor platens were not systematically cleaned (as usually suggested by the sensor vendors).

- the acquired fingerprints were manually analyzed to assure that the maximum rotation is approximately in the range $\left[-15^{\circ}, 15^{\circ}\right]$ and that each pair of impressions of the same finger have a non-null overlapping area.

In Figures 2, 3, 4 and 5 some images from DB1 and DB2 are shown.

As far as DB3 is concerned:

- the fingerprints are from 19 volunteers between ages 5 to 73 (55\% male).

- one-third of the volunteers were over 55 years of age.

- one-third of the volunteers were under 18 years of age.

- one-sixth of the volunteers were under 7 years of age.

- two images of six fingers (thumb, fore and middle on left and right hands) were taken without interleave from each volunteer at each session.

- each volunteer was seen at four sessions, with no more than two sessions on any single day.

- the time between the first and last sessions was at least 3 days and as long as 3 months, depending upon volunteer. 
- the sensor platen was systematically cleaned between acquisition of each image.

- at one session with each volunteer, fingers were cleaned with rubbing alcohol and dried.

- some part of the core was apparent in each image, but care was taken to avoid complete overlap between consecutive images taken during a single session.

- the acquired fingerprints were manually analyzed to assure that the maximum rotation is approximately in the range $\left[-15^{\circ}, 15^{\circ}\right]$ and that each pair of impressions of the same finger have a non-null overlapping area.

Figures 6 and 7 show some sample images taken from DB3.

DB4 needs some explanations. In general, using artificial images for testing biometric systems is not considered a "best practice" [7]. Although this "prejudice" is reasonable for a performance evaluation in real applications, we believe that in a technology evaluation event (like FVC2000) the use of synthetic images has three main advantages:

- it supplies images which are native to none of the participant algorithms, thus providing a fair comparison;

- synthetic fingerprint databases can be created at a very low cost. Acquiring a lot of fingerprints for testing purposes may be problematic due to the great amount of time required and to the privacy legislation which in some countries prohibits the diffusion of such a personal information. Furthermore, once a database has been "used", it expires since for successive fair testing, a new unknown database is mandatory;

- it is possible to adjust the database difficulty by exactly tuning different kinds of perturbations (e.g., maximum rotation and translation, amount of skin distortion, ...).

If the generated artificial images were not a suitable simulation of real fingerprint patterns, the comparisons on the synthetic database would be misleading; on the other hand, the simultaneous presence of three real databases provides a natural way to check the validity of the results on DB4.

The parameters of the synthetic generator were tuned to emulate a low-cost sensor with a small acquisition area; the maximum rotation and displacement and skin-distortion are adjusted to roughly reproduce the perturbations in the three previous databases. Figures 8 and 9 show some sample images taken from DB4. 


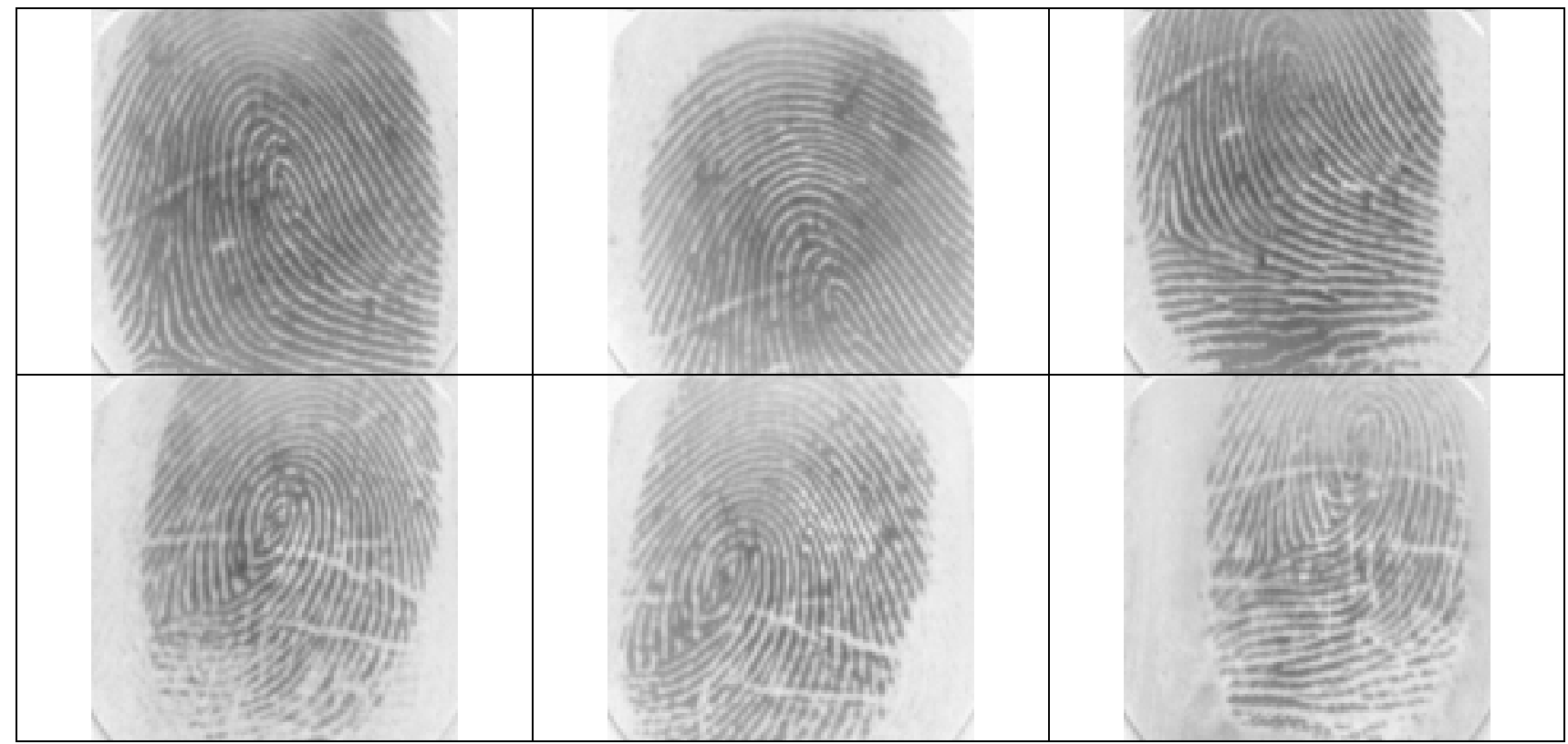

Figure 2. Sample images from DB1; each row shows different impressions of the same finger.

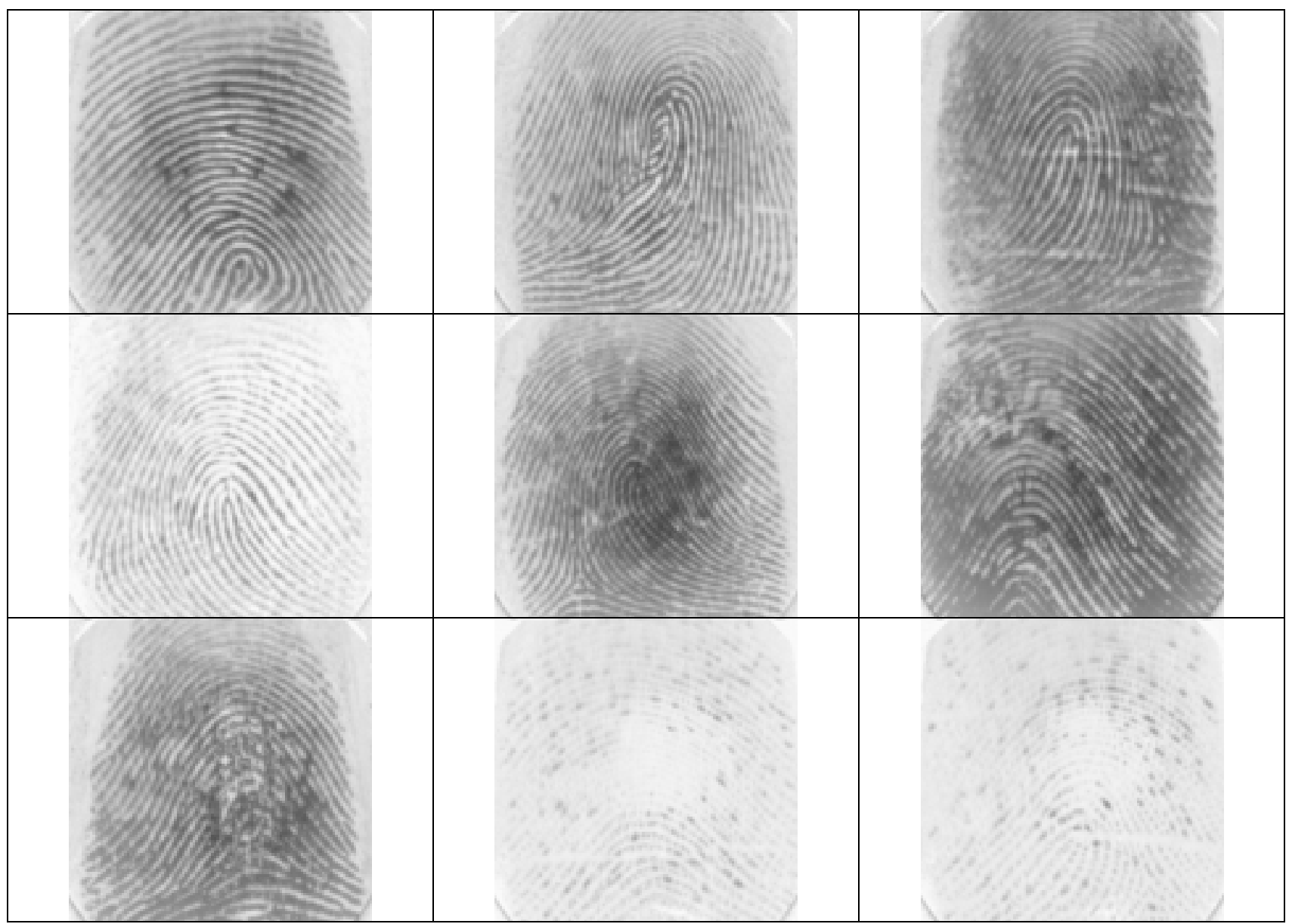

Figure 3. Images from DB1; all the samples are from different fingers and are ordered by quality (top-left: high quality, bottom-right: low quality). 


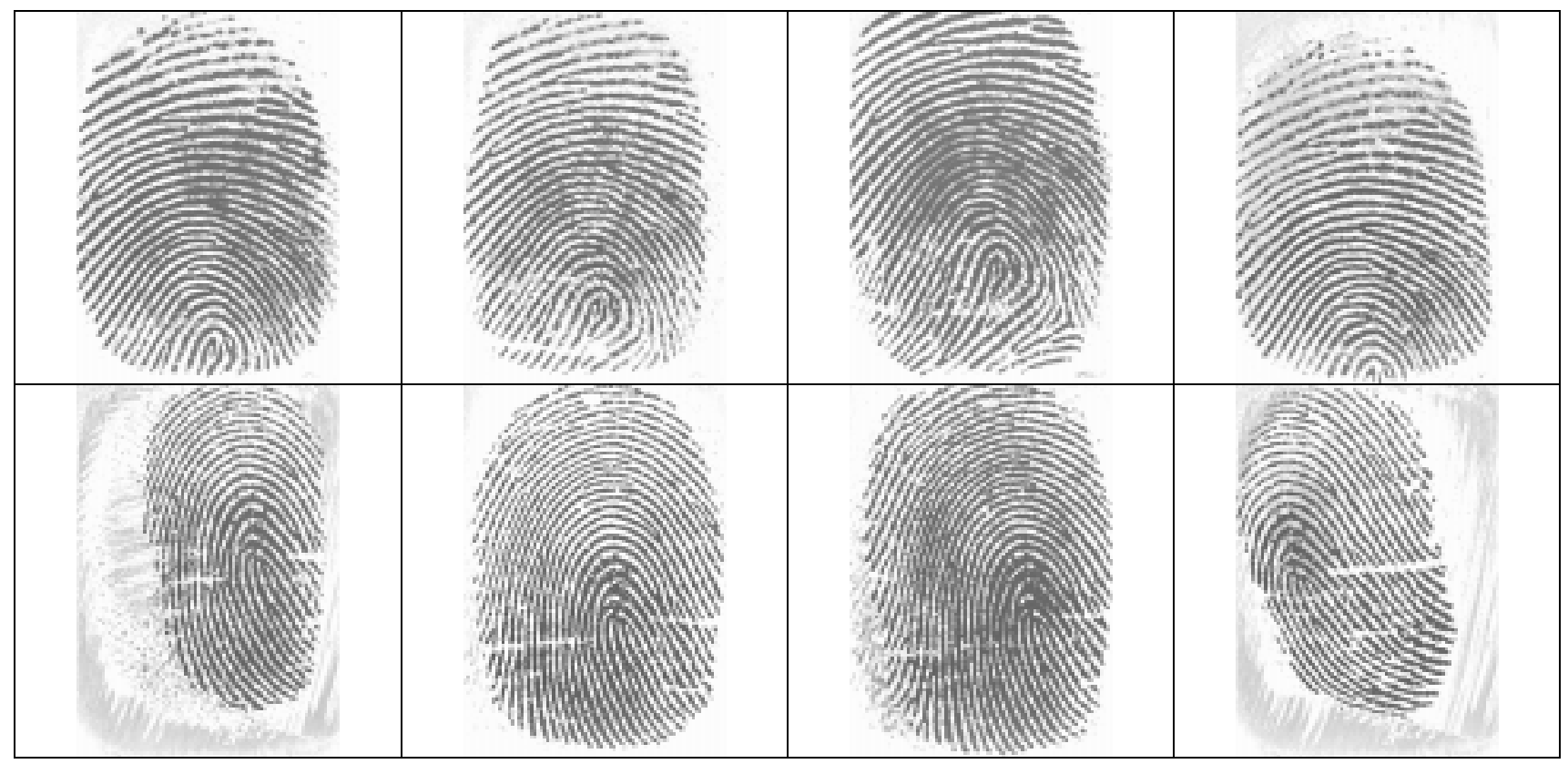

Figure 4. Sample images from DB2; each row shows different impressions of the same finger.

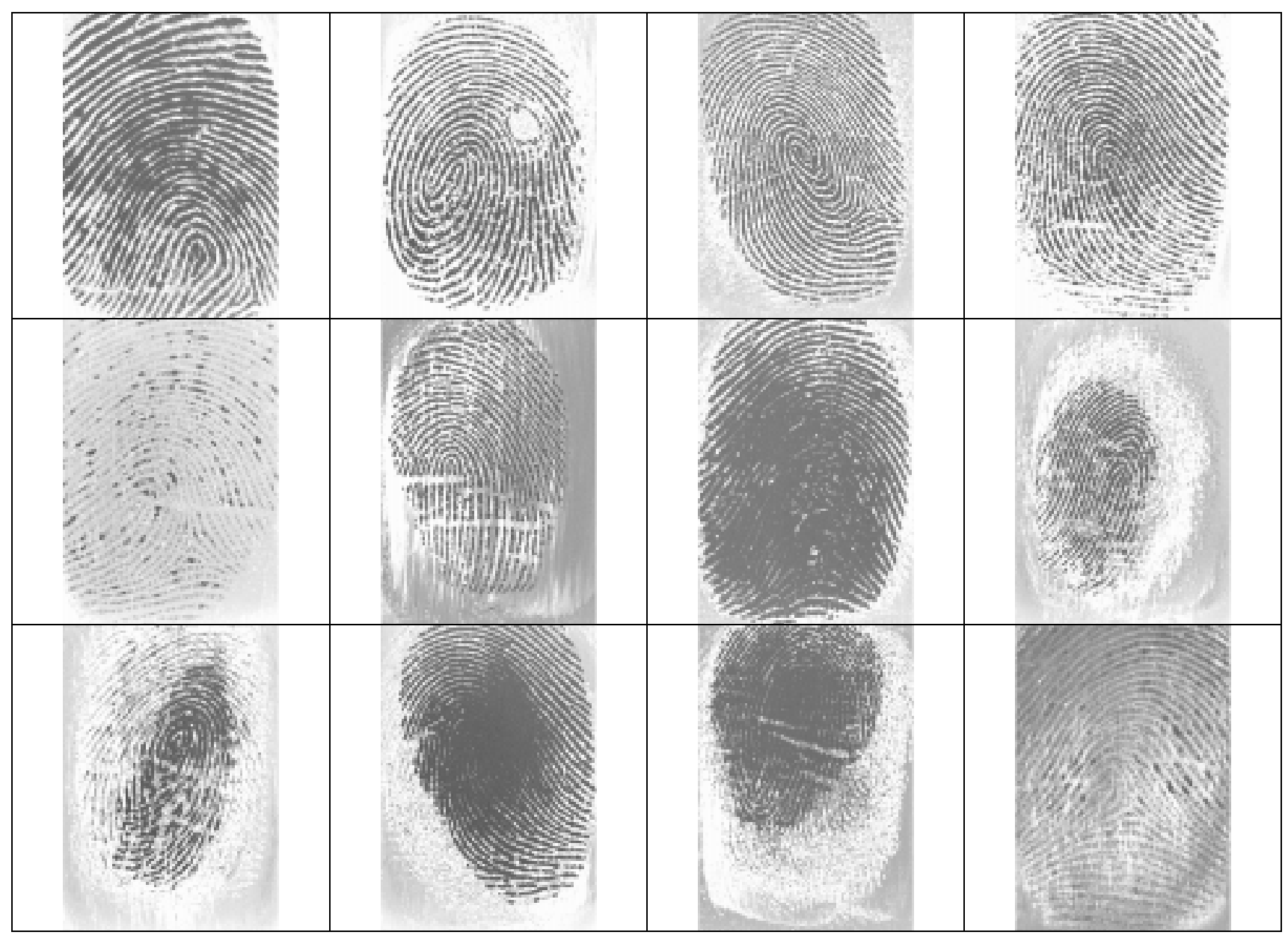

Figure 5. Images from DB2; all the samples are from different fingers and are ordered by quality (top-left: high quality, bottom-right: low quality). 


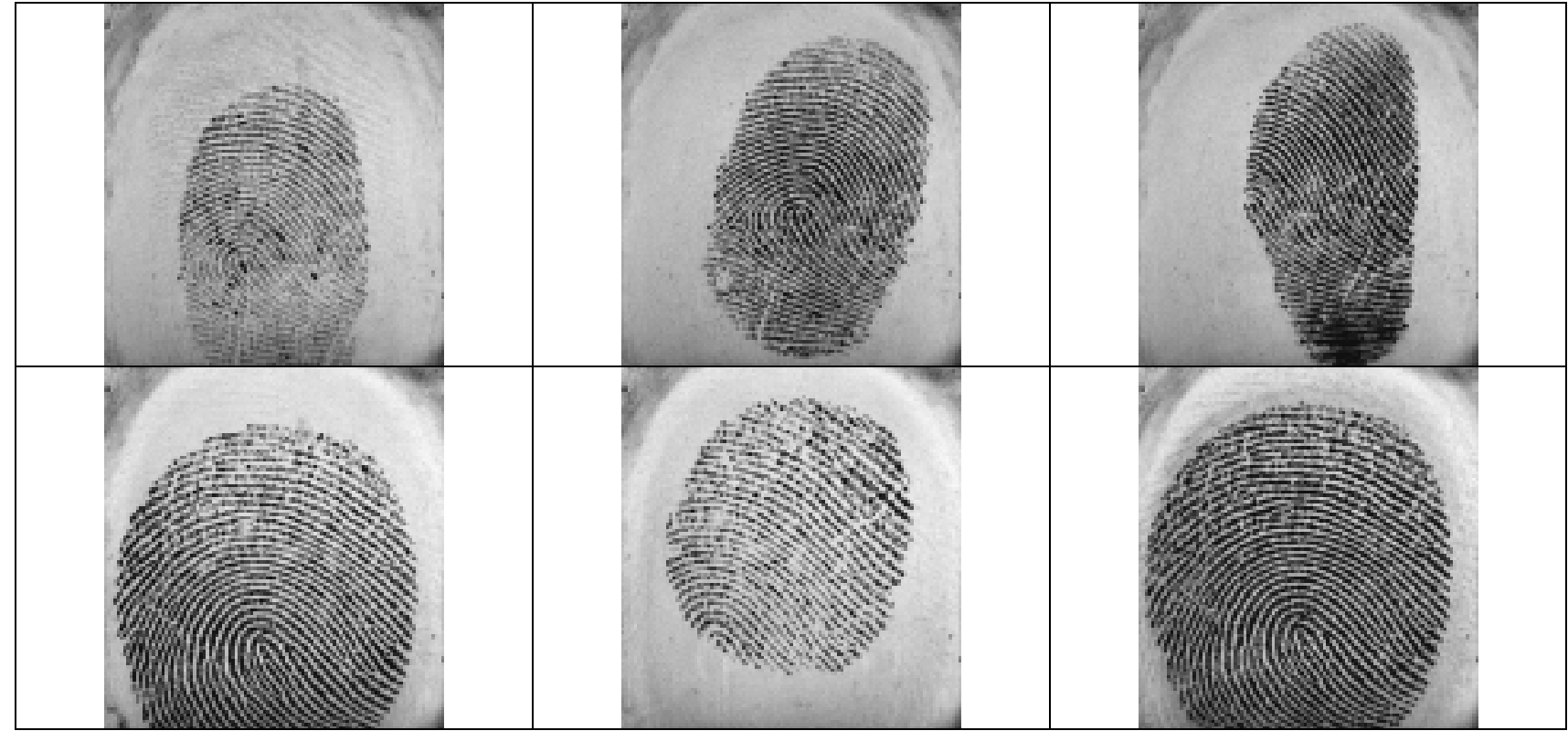

Figure 6. Sample images from DB3; each row shows different impressions of the same finger.

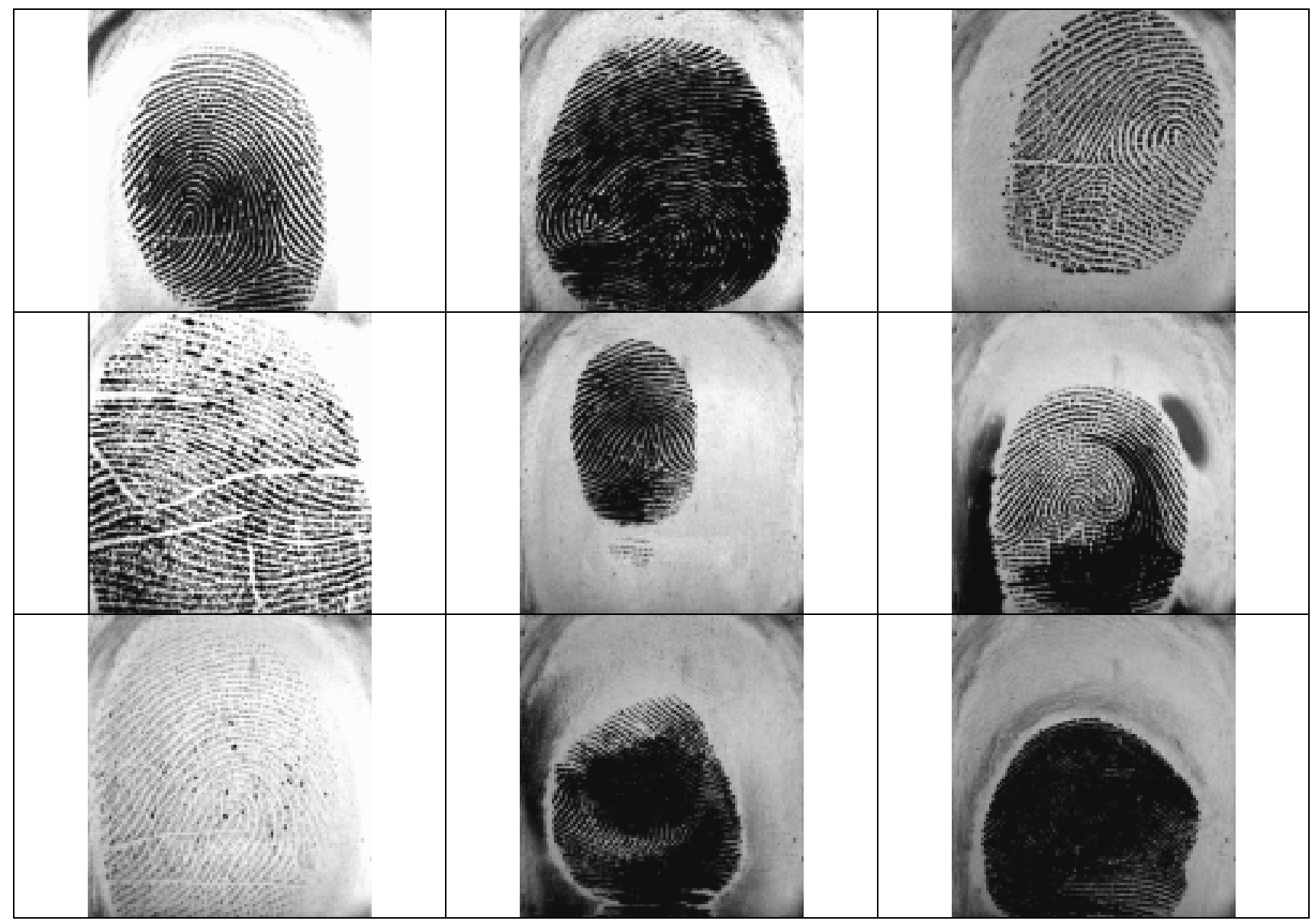

Figure 7. Images from DB3; all the samples are from different fingers and are ordered by quality (top-left: high quality, bottom-right: low quality). 


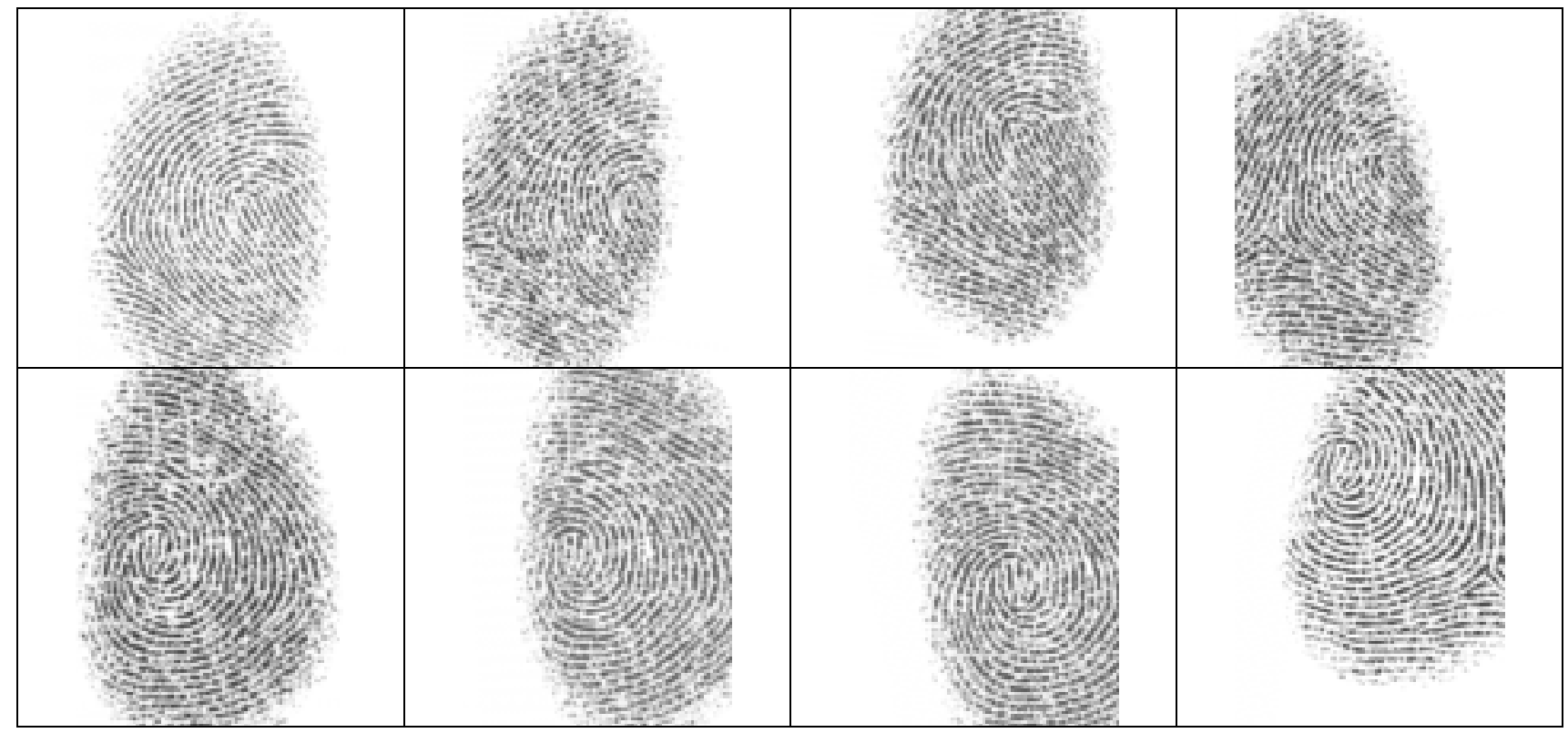

Figure 8. Sample images from DB4; each row shows different impressions of the same finger.

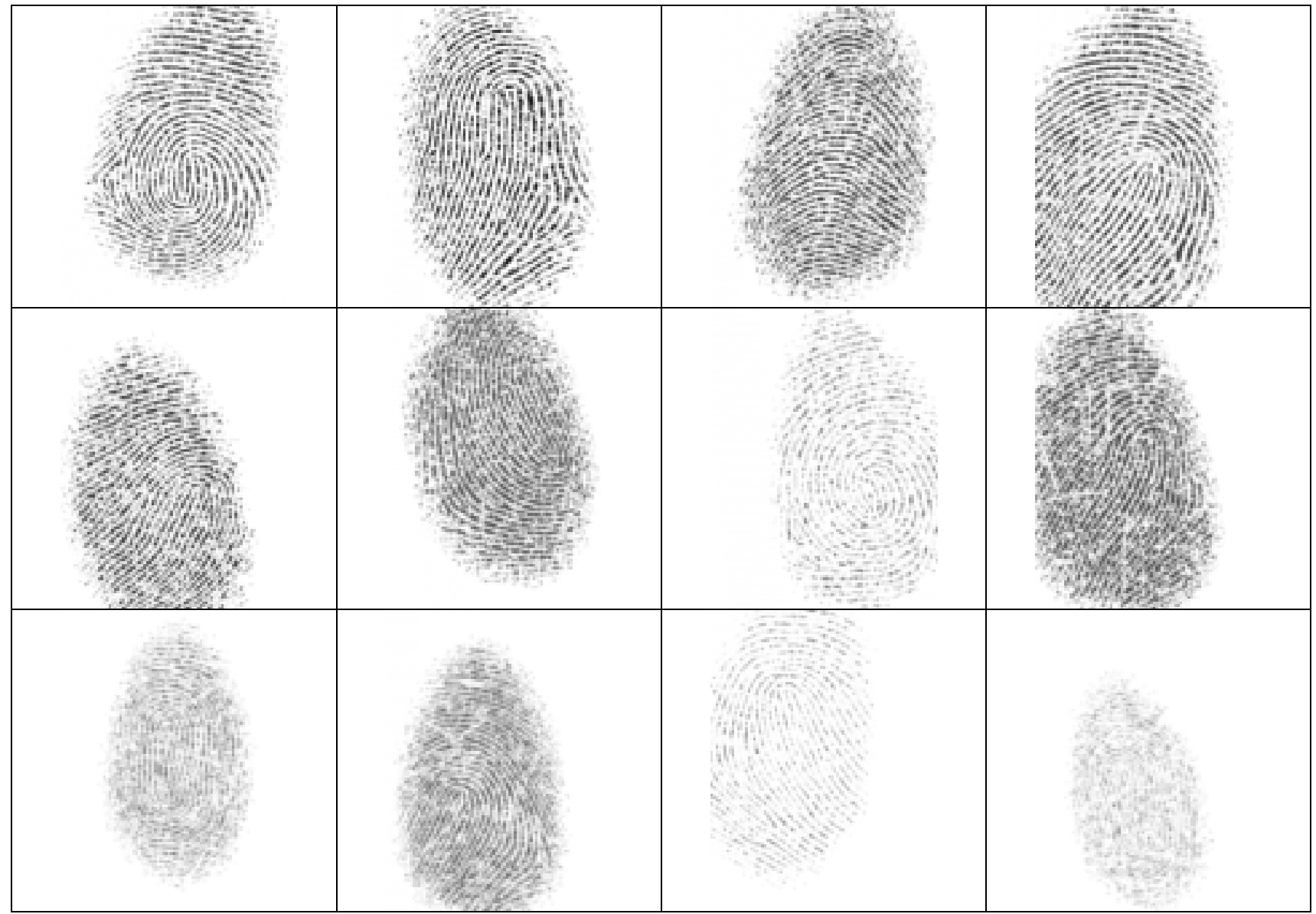

Figure 9. Images from DB4; all the samples are from different fingers and are ordered by quality (top-left: high quality, bottom-right: low quality). 


\section{PERFORMANCE EVALUATION}

In the following, for each database, we will refer to the $j^{\text {th }}$ fingerprint sample of the $i^{\text {th }}$ finger as $\mathrm{F}_{i j}, i=1 . .100, j=1 . .8$ and to the corresponding template (computed from $\mathrm{F}_{i j}$ ) as $\mathrm{T}_{i j}$.

For each database and for each algorithm:

- The templates $\mathrm{T}_{i j}, i=1 . .100, j=1 . .7$ are computed from the corresponding $\mathrm{F}_{i j}$ and stored on disk; one of the following three kinds of rejection can happen for each image $F_{i j}$ :

1. F (Fail): the algorithm declares it cannot enroll the fingerprint image.

2. T (Timeout): the enrollment exceeds the maximum allowed time (15 seconds).

3. $\mathbf{C}$ (Crash): the algorithm crashes during fingerprint processing.

The three types of rejections are singularly summed and their total is stored in $\mathbf{R E} \mathbf{J}_{\text {ENROLL. }}$.

- Each fingerprint template $\mathrm{T}_{i j}$ which has been successfully created in the previous stage is matched against the fingerprint images $\mathrm{F}_{k i}(j<k \leq 8)$ and the corresponding Genuine Matching Scores $\mathbf{g m s}_{i j k}$ are stored". The number of matches (denoted as NGRA - Number of Genuine Recognition Attempts $)$ is $((8 \times 7) / 2) \times 100=2,800$ in case $\mathbf{R E} \mathbf{J}_{\text {ENROLL }}=0$. It is possible to have Fail, Timeout (5 seconds) or Crash rejections. These events are accumulated into REJ $\mathbf{J}_{\mathbf{N G R A}}$; no $\mathbf{g m s}_{i j k}$ is stored in this case.

- Each fingerprint template $\mathrm{T}_{1 i}, i=1 . .100$ is matched against the first fingerprint image from different fingers $\mathrm{F}_{1 k}(i<k \leq 100)$ and the corresponding Impostor Matching Scores ims $_{i k}$ are stored. The number of matches (denoted as NIRA - Number of Impostor Recognition Attempts) is $((100 \times 99) / 2)=4,950$ in case $\mathbf{R E} \mathbf{J}_{\mathbf{E N R O L L}}=0$. It is possible to have Fail, Timeout (5 seconds) or Crash rejections. These events are accumulated into $\mathbf{R E} \mathbf{J}_{\mathbf{N I R A}}$; no ims $\mathbf{i}_{i k}$ is stored in this case.

- The genuine matching score distribution and the impostor matching score distribution are computed and graphically reported to show how the algorithm "separates" the two classes. In fingerprinting, higher scores are associated with more closely matching images.

- The $\operatorname{FMR}(t)$ (False Match Rate) and FNMR(t) (False Non-Match Rate) curves are computed from the above distributions for $t$ ranging from 0 to $1^{\dagger}$. Given a threshold $t, \mathbf{F M R}(t)$ denotes the percentage of $\mathbf{i m s}_{i k} \geq t$, whereas $\mathbf{F N M R}(t)$ denotes the percentage of $\mathbf{g m s}_{i j k}<t$. Actually, since

\footnotetext{
* If the matching $g$ against $h$ is performed, the symmetric one (i.e., $h$ against $g$ ) is not executed to avoid correlation.

$\dagger$ FMR and FNMR are often referred as FAR (False Acceptance Rate) and FRR (False Rejection Rate) respectively, but the FAR/FRR notation is misleading in some applications. For example, in a welfare benefits system, which uses fingerprint identification to prevent multiple concessions under false identity, the system "falsely accepts" an applicant if his/her fingerprint is "falsely rejected"; otherwise, a "false acceptance" causes a "false rejection".
} 
FMR and FNMR are used in the contest to compare the performance of different algorithms,

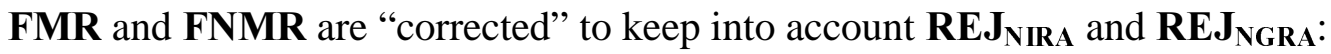

$$
\boldsymbol{F M R}(t)=\frac{\operatorname{card}\left\{\mathbf{i m s}_{i k} \mid \mathbf{i m s}_{i k} \geq t\right\}}{\text { NIRA }}, \operatorname{FNMR}(t)=\frac{\operatorname{card}\left\{\mathbf{g m s}_{i j k} \mid \mathbf{g m s}_{i j k}<t\right\}+\mathbf{R E} \mathbf{J}_{\mathbf{N G R A}}}{\mathbf{N G R A}}
$$

This correction assumes that a failure to match is always treated by the system as a "non match" (matching score $<0$ ).

- A ROC (Receiving Operating Curve) is given where FNMR is plotted as a function of FMR; the curve is drawn in log-log scales for better comprehension.

- The Equal Error Rate EER is computed as the point where $\operatorname{FNMR}(t)=\mathbf{F M R}(t)$; in practice the score distributions are not continuous and a crossover point might not exist. In this case, we report the interval $\left[\mathbf{E} \mathbf{E} \mathbf{R}_{\text {low }}, \mathbf{E E R}_{\text {high }}\right]$ :

$$
\begin{gathered}
t_{1}=\underset{t \in\left\{\mathbf{g m s}_{i j k}\right\} \cup\left\{\mathbf{i m s}_{i k}\right\}}{\max }\{t \mid \mathbf{F N M R}(t) \leq \mathbf{F M R}(t)\}, \quad t_{2}=\underset{t \in\left\{\mathbf{g m s}_{i j k}\right\} \cup\left\{\mathbf{m m s}_{i k}\right\}}{\min }\{t \mid \mathbf{F N M R}(t) \geq \mathbf{F M R}(t)\} \\
{\left[\mathbf{E E R}_{\text {low }}, \mathbf{E E R}_{\text {high }}\right]=\left\{\begin{array}{l}
{\left[\mathbf{F N M R}\left(t_{1}\right), \mathbf{F M R}\left(t_{1}\right)\right] \quad \text { if } \mathbf{F N M R}\left(t_{1}\right)+\mathbf{F M R}\left(t_{1}\right) \leq \mathbf{F M R}\left(t_{2}\right)+\mathbf{F N M R}\left(t_{2}\right)} \\
{\left[\mathbf{F M R}\left(t_{2}\right), \mathbf{F N M R}\left(t_{2}\right)\right]}
\end{array}\right.}
\end{gathered}
$$

and we estimate EER as $\left(\mathbf{E E R} \mathbf{R}_{\text {low }}+\mathbf{E E} \mathbf{R}_{\text {high }}\right) / 2$ (see Fig. 10).

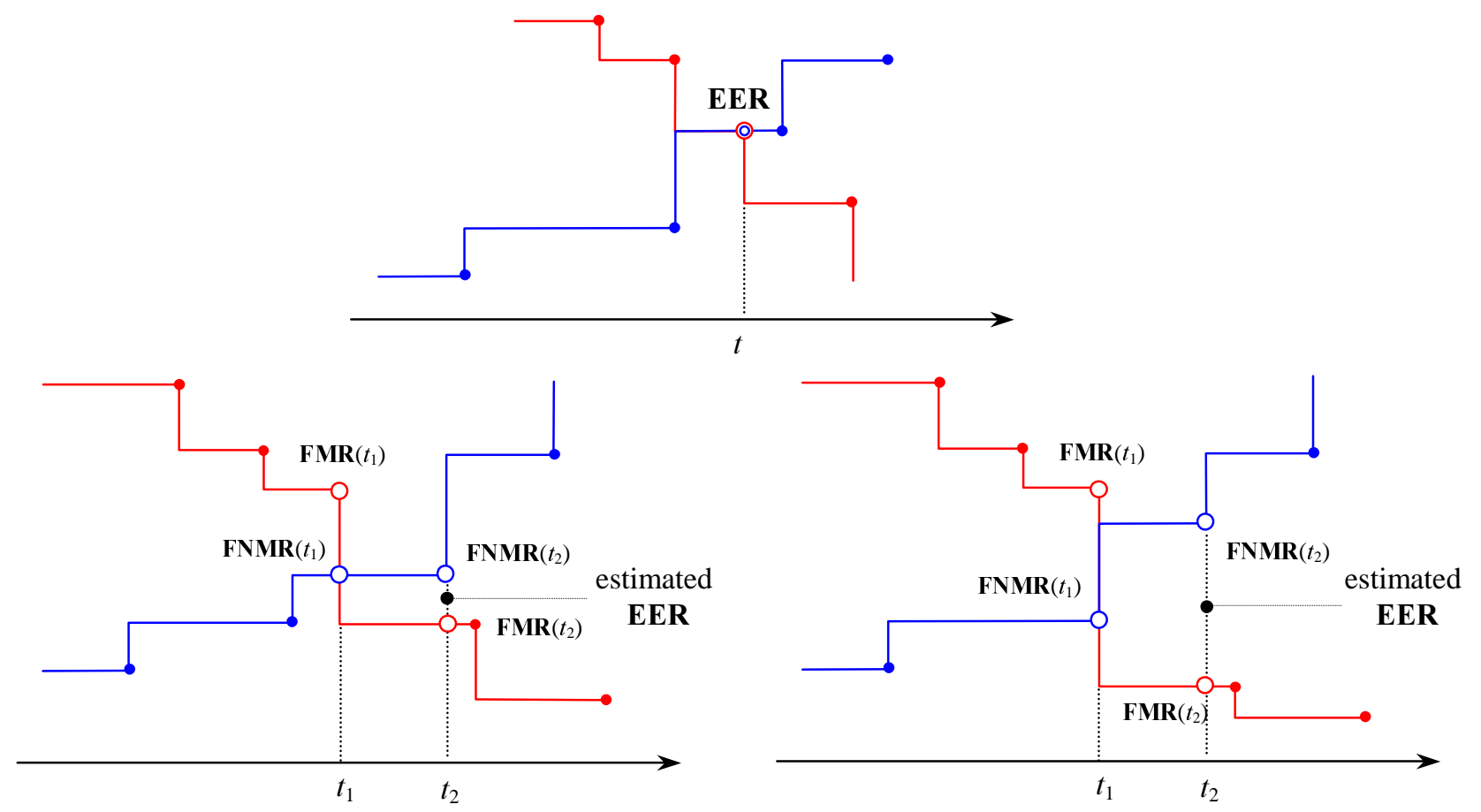

Figure 10. On the top an example is given where EER point exists. On the bottom two cases are shown where EER does not exist and the corresponding intervals are highlighted. 
- EER $^{*}$ is computed as the value that EER would take if the matching failures (REJ $\mathbf{J}_{\mathbf{N G R A}}$ and REJ $\mathbf{J}_{\text {NIRA }}$ ) were excluded from the computation of False Match and False Non-Match rates:

$$
\mathbf{F M R}^{*}(t)=\frac{\operatorname{card}\left\{\mathbf{i m s}_{i k} \mid \mathbf{i m s}_{i k} \geq t\right\}}{\mathbf{N I R A}-\mathbf{R E J}_{\mathbf{N I R A}}}, \quad \mathbf{F N M R}^{*}(t)=\frac{\operatorname{card}\left\{\mathbf{g m s}_{i j k} \mid \mathbf{g m s}_{i j k}<t\right\}}{\mathbf{N G R A}-\mathbf{R E J}_{\mathbf{N G R A}}}
$$

- ZeroFMR is given as the lowest FNMR at which no False Matches occur and ZeroFNMR as the lowest FMR at which no False Non-Matches occur:

$$
\operatorname{ZeroFMR}(t)=\min _{t}\{\mathbf{F N M R}(t) \mid \mathbf{F M R}(t)=0\}, \operatorname{ZeroFNMR}(t)=\min _{t}\{\mathbf{F M R}(t) \mid \operatorname{FNMR}(t)=0\}
$$

Both ZeroFMR and ZeroFNMR may not exist; in such a case we assign to them the value 1.

- The average enroll time is calculated as the average CPU time taken by a single enrollment operation, and average match time as the average CPU time taken by a single match operation between a template and a fingerprint image.

\section{RESULTS}

This section reports the performance of the tested algorithms on each separate database (Figures 11, 12, 13, 14 and Tables III, IV, V, VI) and the average results over the four databases (Table VII). The notation introduced in Section 4 is used in both the graphics and tables, with the only exception

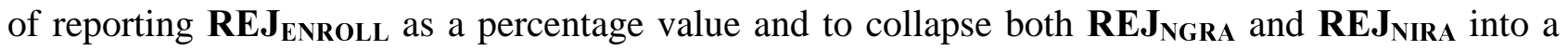
single percentage value $\mathbf{R E} \mathbf{J}_{\mathbf{M A T C H}}$ :

$$
\text { REJ }_{\text {MATCH }}=\frac{\text { NIRA } \cdot \text { REJ }_{\text {NIRA }}+\text { NGRA } \cdot \text { REJ }_{\text {NGRA }}}{\text { NIRA }+ \text { NGRA }}
$$

It is worth noting that, for a correct interpretation of the results, EER alone is not an exhaustive metric, but REJ $\mathbf{J}_{\text {ENROLL }}$ should be also taken into account.

For each algorithm, detailed results (including genuine and impostor distributions, FMR and FNMR curves, NGRA, NIRA, ...) are reported in Appendix A. 


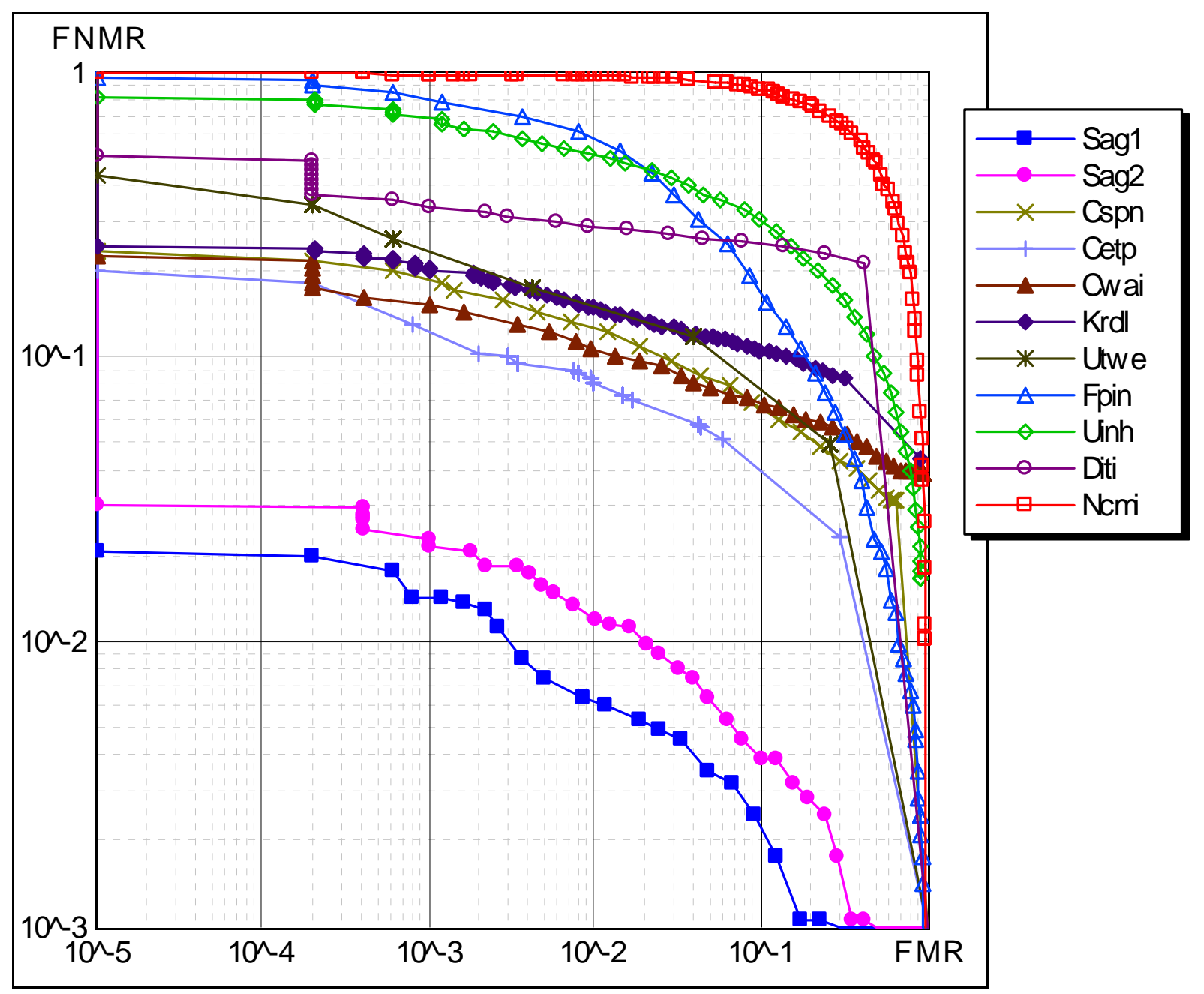

Figure 11. ROC curves on DB1.

\begin{tabular}{|c|c|c|c|c|c|c|}
\hline Algorithm & EER & EER* & REJ $_{\text {ENROLL }}$ & REJ $_{\text {MATCH }}$ & $\begin{array}{l}\text { Avg Enroll } \\
\text { Time }\end{array}$ & $\begin{array}{l}\text { Avg Match } \\
\text { Time }\end{array}$ \\
\hline Sagl & $0.67 \%$ & $0.67 \%$ & $0.00 \%$ & $0.00 \%$ & $2.48 \mathrm{sec}$ & $0.96 \mathrm{sec}$ \\
\hline Sag2 & $1.17 \%$ & $1.17 \%$ & $0.00 \%$ & $0.00 \%$ & $0.88 \mathrm{sec}$ & $0.88 \mathrm{sec}$ \\
\hline Cetp & $5.06 \%$ & $5.06 \%$ & $0.00 \%$ & $0.00 \%$ & $0.81 \mathrm{sec}$ & $0.89 \mathrm{sec}$ \\
\hline Cwai & $7.06 \%$ & $4.27 \%$ & $3.71 \%$ & $3.90 \%$ & $0.22 \mathrm{sec}$ & $0.32 \mathrm{sec}$ \\
\hline Cspn & $7.60 \%$ & $7.60 \%$ & $0.00 \%$ & $0.00 \%$ & $0.17 \mathrm{sec}$ & $0.17 \mathrm{sec}$ \\
\hline Utwe & $7.98 \%$ & $7.98 \%$ & $0.00 \%$ & $0.00 \%$ & $10.40 \mathrm{sec}$ & $2.10 \mathrm{sec}$ \\
\hline Krdl & $10.66 \%$ & $7.35 \%$ & $6.43 \%$ & $6.59 \%$ & $1.00 \mathrm{sec}$ & $1.06 \mathrm{sec}$ \\
\hline Fpin & $13.46 \%$ & $13.46 \%$ & $0.00 \%$ & $0.00 \%$ & $0.83 \mathrm{sec}$ & $0.87 \mathrm{sec}$ \\
\hline Uinh & $21.02 \%$ & $20.65 \%$ & $1.71 \%$ & $5.08 \%$ & $0.53 \mathrm{sec}$ & $0.56 \mathrm{sec}$ \\
\hline Diti & $23.63 \%$ & $23.63 \%$ & $0.00 \%$ & $0.00 \%$ & $0.65 \mathrm{sec}$ & $0.72 \mathrm{sec}$ \\
\hline Ncmi & $49.11 \%$ & $49.15 \%$ & $0.00 \%$ & $0.12 \%$ & $1.13 \mathrm{sec}$ & $1.34 \mathrm{sec}$ \\
\hline
\end{tabular}

Table III. Algorithm performance over DB1 sorted by EER. 


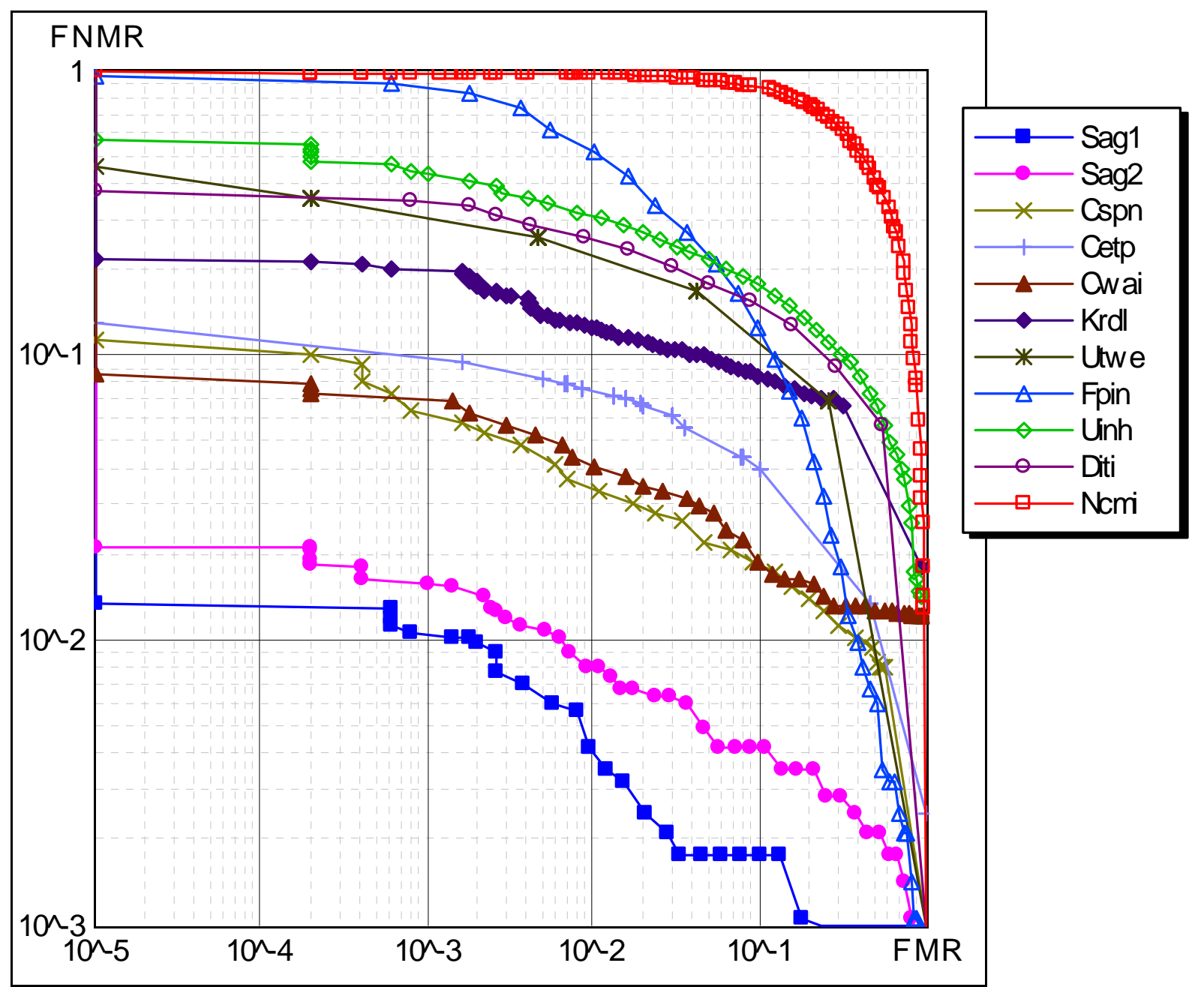

Figure 12. ROC curves on DB2.

\begin{tabular}{|c|c|c|c|c|c|c|}
\hline Algorithm & EER & EER* & REJ $_{\text {ENROLL }}$ & REJ $_{\text {MATCH }}$ & $\begin{array}{l}\text { Avg Enroll } \\
\text { Time }\end{array}$ & $\begin{array}{l}\text { Avg Match } \\
\text { Time }\end{array}$ \\
\hline Sag1 & $0.61 \%$ & $0.61 \%$ & $0.00 \%$ & $0.00 \%$ & $2.63 \mathrm{sec}$ & $1.03 \mathrm{sec}$ \\
\hline Sag2 & $0.82 \%$ & $0.82 \%$ & $0.00 \%$ & $0.00 \%$ & $0.93 \mathrm{sec}$ & $0.93 \mathrm{sec}$ \\
\hline Cspn & $2.75 \%$ & $2.75 \%$ & $0.00 \%$ & $0.00 \%$ & $0.17 \mathrm{sec}$ & $0.17 \mathrm{sec}$ \\
\hline Cwai & $3.01 \%$ & $2.16 \%$ & $1.29 \%$ & $1.29 \%$ & $0.23 \mathrm{sec}$ & $0.30 \mathrm{sec}$ \\
\hline Cetp & $4.63 \%$ & $4.51 \%$ & $0.00 \%$ & $0.09 \%$ & $0.85 \mathrm{sec}$ & $0.98 \mathrm{sec}$ \\
\hline$K r d l$ & $8.83 \%$ & $7.53 \%$ & $3.29 \%$ & $4.41 \%$ & $1.16 \mathrm{sec}$ & $2.88 \mathrm{sec}$ \\
\hline Utwe & $10.65 \%$ & $10.65 \%$ & $0.00 \%$ & $0.00 \%$ & $10.42 \mathrm{sec}$ & $2.12 \mathrm{sec}$ \\
\hline Fpin & $11.14 \%$ & $11.14 \%$ & $0.00 \%$ & $0.00 \%$ & $1.16 \mathrm{sec}$ & $1.24 \mathrm{sec}$ \\
\hline Diti & $13.83 \%$ & $13.83 \%$ & $0.00 \%$ & $0.00 \%$ & $1.21 \mathrm{sec}$ & $1.28 \mathrm{sec}$ \\
\hline Uinh & $15.22 \%$ & $14.70 \%$ & $0.86 \%$ & $4.08 \%$ & $0.60 \mathrm{sec}$ & $0.65 \mathrm{sec}$ \\
\hline Ncmi & $46.15 \%$ & $46.15 \%$ & $0.00 \%$ & $0.00 \%$ & $1.28 \mathrm{sec}$ & $1.57 \mathrm{sec}$ \\
\hline
\end{tabular}

Table IV. Algorithm performance over DB2 sorted by EER. 


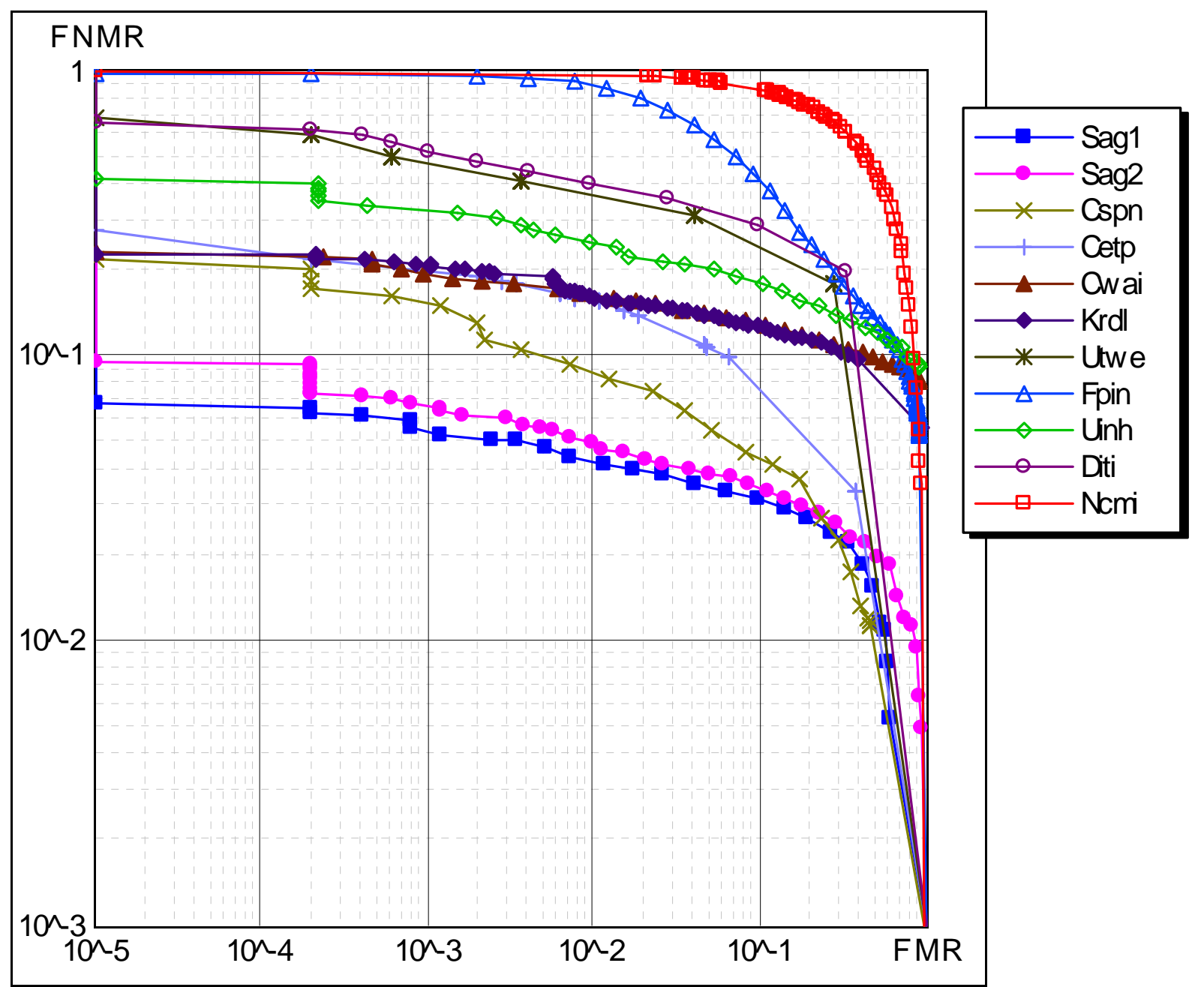

Figure 13. ROC curves on DB3.

\begin{tabular}{|c|c|c|c|c|c|c|}
\hline Algorithm & EER & EER* & REJ $J_{\text {ENROLL }}$ & REJ $_{\text {MATCH }}$ & $\begin{array}{c}\text { Avg Enroll } \\
\text { Time }\end{array}$ & $\begin{array}{l}\text { Avg Match } \\
\text { Time }\end{array}$ \\
\hline Sagl & $3.64 \%$ & $3.64 \%$ & $0.00 \%$ & $0.00 \%$ & $5.70 \mathrm{sec}$ & $2.13 \mathrm{sec}$ \\
\hline Sag2 & $4.01 \%$ & $4.01 \%$ & $0.00 \%$ & $0.00 \%$ & $1.94 \mathrm{sec}$ & $1.94 \mathrm{sec}$ \\
\hline Cspn & $5.36 \%$ & $5.33 \%$ & $0.57 \%$ & $1.24 \%$ & $0.35 \mathrm{sec}$ & $0.36 \mathrm{sec}$ \\
\hline Cetp & $8.29 \%$ & $8.29 \%$ & $0.00 \%$ & $0.00 \%$ & $1.49 \mathrm{sec}$ & $1.66 \mathrm{sec}$ \\
\hline Cwai & $11.94 \%$ & $5.90 \%$ & $12.86 \%$ & $8.00 \%$ & $0.46 \mathrm{sec}$ & $0.57 \mathrm{sec}$ \\
\hline$K r d l$ & $12.20 \%$ & $8.03 \%$ & $6.86 \%$ & $5.12 \%$ & $1.48 \mathrm{sec}$ & $1.60 \mathrm{sec}$ \\
\hline Uinh & $16.32 \%$ & $10.14 \%$ & $10.29 \%$ & $7.64 \%$ & $1.28 \mathrm{sec}$ & $1.36 \mathrm{sec}$ \\
\hline Utwe & $17.73 \%$ & $17.73 \%$ & $0.00 \%$ & $0.00 \%$ & $10.44 \mathrm{sec}$ & $2.31 \mathrm{sec}$ \\
\hline Diti & $22.63 \%$ & $22.63 \%$ & $0.00 \%$ & $0.00 \%$ & $2.59 \mathrm{sec}$ & $2.67 \mathrm{sec}$ \\
\hline Fpin & $23.18 \%$ & $23.18 \%$ & $0.00 \%$ & $0.00 \%$ & $2.13 \mathrm{sec}$ & $2.19 \mathrm{sec}$ \\
\hline Ncmi & $47.43 \%$ & $47.44 \%$ & $0.00 \%$ & $0.01 \%$ & $2.25 \mathrm{sec}$ & $2.75 \mathrm{sec}$ \\
\hline
\end{tabular}

Table V. Algorithm performance over DB3 sorted by EER. 


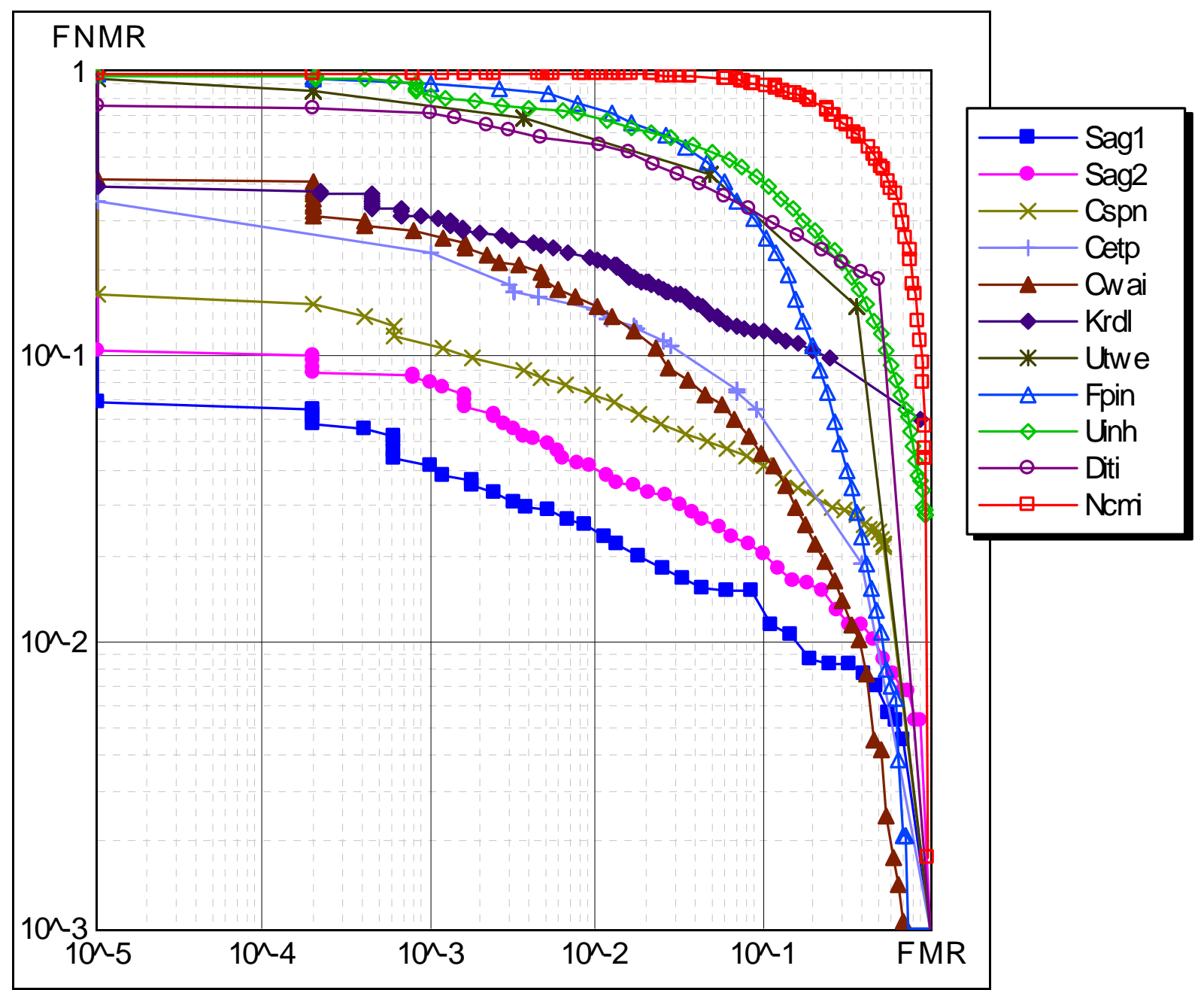

Figure 14. ROC curves on DB4.

\begin{tabular}{|c|c|c|c|c|c|c|}
\hline Algorithm & EER & EER* & REJ $_{\text {ENROLL }}$ & REJ $_{\text {MATCH }}$ & $\begin{array}{l}\text { Avg Enroll } \\
\text { Time }\end{array}$ & $\begin{array}{l}\text { Avg Match } \\
\text { Time }\end{array}$ \\
\hline Sag1 & $1.99 \%$ & $1.99 \%$ & $0.00 \%$ & $0.00 \%$ & $1.90 \mathrm{sec}$ & $0.77 \mathrm{sec}$ \\
\hline Sag2 & $3.11 \%$ & $3.11 \%$ & $0.00 \%$ & $0.00 \%$ & $0.69 \mathrm{sec}$ & $0.69 \mathrm{sec}$ \\
\hline Cspn & $5.04 \%$ & $5.04 \%$ & $0.00 \%$ & $0.00 \%$ & $0.11 \mathrm{sec}$ & $0.11 \mathrm{sec}$ \\
\hline Cwai & $6.30 \%$ & $6.30 \%$ & $0.00 \%$ & $0.00 \%$ & $0.16 \mathrm{sec}$ & $0.20 \mathrm{sec}$ \\
\hline Cetp & $7.29 \%$ & $7.29 \%$ & $0.00 \%$ & $0.00 \%$ & $0.65 \mathrm{sec}$ & $0.72 \mathrm{sec}$ \\
\hline$K r d l$ & $12.08 \%$ & $7.46 \%$ & $10.86 \%$ & $10.24 \%$ & $0.70 \mathrm{sec}$ & $0.79 \mathrm{sec}$ \\
\hline Fpin & $16.00 \%$ & $16.00 \%$ & $0.00 \%$ & $0.00 \%$ & $0.77 \mathrm{sec}$ & $0.80 \mathrm{sec}$ \\
\hline Diti & $23.80 \%$ & $23.80 \%$ & $0.00 \%$ & $0.00 \%$ & $0.52 \mathrm{sec}$ & $0.60 \mathrm{sec}$ \\
\hline Utwe & $24.59 \%$ & $24.59 \%$ & $0.00 \%$ & $0.00 \%$ & $10.42 \mathrm{sec}$ & $4.17 \mathrm{sec}$ \\
\hline Uinh & $24.77 \%$ & $23.74 \%$ & $2.14 \%$ & $4.28 \%$ & $0.42 \mathrm{sec}$ & $0.45 \mathrm{sec}$ \\
\hline Ncmi & $48.67 \%$ & $48.77 \%$ & $0.00 \%$ & $0.25 \%$ & $1.08 \mathrm{sec}$ & $1.19 \mathrm{sec}$ \\
\hline
\end{tabular}

Table VI. Algorithm performance over DB4 sorted by EER. 


\begin{tabular}{||c|c|c|c|c|c|c|}
\hline Algorithm & $\begin{array}{c}\text { Avg } \\
\text { EER }\end{array}$ & $\begin{array}{c}\text { Avg } \\
\text { EER* }\end{array}$ & $\begin{array}{c}\text { Avg } \\
\text { REJ }\end{array}$ & $\begin{array}{c}\text { Avg } \\
\text { REJ }\end{array}$ & $\begin{array}{c}\text { Avg Enroll } \\
\text { Time }\end{array}$ & $\begin{array}{c}\text { Avg Match } \\
\text { Time }\end{array}$ \\
\hline Sag1 & $1.73 \%$ & $1.73 \%$ & $0.00 \%$ & $0.00 \%$ & $3.18 \mathrm{sec}$ & $1.22 \mathrm{sec}$ \\
\hline Sag2 & $2.28 \%$ & $2.28 \%$ & $0.00 \%$ & $0.00 \%$ & $1.11 \mathrm{sec}$ & $1.11 \mathrm{sec}$ \\
\hline Csph & $5.19 \%$ & $5.18 \%$ & $0.14 \%$ & $0.31 \%$ & $0.20 \mathrm{sec}$ & $0.20 \mathrm{sec}$ \\
\hline Cetp & $6.32 \%$ & $6.29 \%$ & $0.00 \%$ & $0.02 \%$ & $0.95 \mathrm{sec}$ & $1.06 \mathrm{sec}$ \\
\hline Cwai & $7.08 \%$ & $4.66 \%$ & $4.46 \%$ & $3.14 \%$ & $0.27 \mathrm{sec}$ & $0.35 \mathrm{sec}$ \\
\hline Krdl & $10.94 \%$ & $7.59 \%$ & $6.86 \%$ & $6.52 \%$ & $1.08 \mathrm{sec}$ & $1.58 \mathrm{sec}$ \\
\hline Utwe & $15.24 \%$ & $15.24 \%$ & $0.00 \%$ & $0.00 \%$ & $10.42 \mathrm{sec}$ & $2.67 \mathrm{sec}$ \\
\hline Fpin & $15.94 \%$ & $15.94 \%$ & $0.00 \%$ & $0.00 \%$ & $1.22 \mathrm{sec}$ & $1.27 \mathrm{sec}$ \\
\hline Uinh & $19.33 \%$ & $17.31 \%$ & $3.75 \%$ & $5.23 \%$ & $0.71 \mathrm{sec}$ & $0.76 \mathrm{sec}$ \\
\hline Diti & $20.97 \%$ & $20.97 \%$ & $0.00 \%$ & $0.00 \%$ & $1.24 \mathrm{sec}$ & $1.32 \mathrm{sec}$ \\
\hline Ncmi & $47.84 \%$ & $47.88 \%$ & $0.00 \%$ & $0.09 \%$ & $1.44 \mathrm{sec}$ & $1.71 \mathrm{sec}$ \\
\hline
\end{tabular}

Table VII. Average performance over the four databases sorted by Avg EER.

\section{CONCLUSIONS}

Most of the algorithms submitted to the competition performed well, if we keep into account the difficulty of adapting existent techniques to new kind of images. In particular, algorithms Sagl and Sag2 proved to be very accurate and Cspn exhibited a good tradeoff between accuracy and efficiency.

Databases DB1 and DB2 proved to be "easier" than DB3, even though the sensor used for DB3 is of higher quality. This means that the acquisition conditions and the volunteer population can have a stronger impact on the performance than sensor quality.

The synthetically generated database (DB4) was demonstrated to be adequate to FVC2000 purposes: in particular, from Tables III, IV, V and VI, it is evident that the algorithm ranking on DB4 is quite similar to the other databases. That is, if an algorithm performs well on real fingerprints, then it also performs well on synthetic fingerprints and vice-versa. The visual analysis of impostor and genuine distributions (Appendix A) definitely support this claim, since no significant differences are seen between the DB4 graphics and the others.

Once again we would like to remark that the obtained results do not necessarily reflect the performance that the participating algorithms would achieve in a real environment or when embedded into a complete biometric system. In any event, we believe that FVC2000 results:

- give a useful overview of the state-of-the-art in this field

- allow researchers and companies to test their algorithms over common databases

- provide guidance to the participants for improving their algorithms

In the future, we intend to continue supporting this initiative as follows: 
- A web site will be setup containing FVC2000 results and technical reports

- Companies and academic research groups will be allowed to test new algorithms or improved version of existing algorithms on the FVC2000 benchmark database and to add their results to the FVC2000 web site. The new "entries" will be kept isolated from the original participants since hereinafter the full databases are known in advance, which could allow algorithm tuning to give unfair advantage to newer entrants.

- New databases for a new benchmarking test will be eventually collected.

- The feasibility of generating synthetic databases for future technology evaluations will be further investigated.

\section{REFERENCES}

[1] A. Jain, R. Bolle and S. Pankanti, Biometrics - Personal Identification in Networked Society, Kluwer Academic Publisher, 1999.

[2] C. I. Watson and C. L. Wilson, "NIST Special Database 4, Fingerprint Database", U.S. National Institute of Standards and Technology, 1992.

[3] C.I. Watson, "NIST Special Database 14, Fingerprint Database", U.S. National Institute of Standards and Technology, 1993.

[4] C.I. Watson, "NIST Special Standard Reference Database 24, NIST Digital Video of LiveScan Fingerprint Database", U.S. National Institute of Standards and Technology, 1998.

[5] J.L. Wayman, "Technical Testing and Evaluation of Biometric Devices", in A. Jain, et. al, Biometrics - Personal Identification in Networked Society, Kluwer Academic Publisher, 1999.

[6] P.J.Phillips, A. Martin, C.L. Wilson and M. Przybocky, "An Introduction to Evaluating Biometric Systems”, IEEE Computer Magazine, February 2000.

[7] UK Government's Biometrics Working Group, "Best Practices in Testing and Reporting Performance of Biometric Devices", January 2000 (available on-line at: http://www.afb.org.uk/bwg/bestprac10.pdf).

[8] BIOTEST: European Esprit Project No: 21978 (http://www.npl.co.uk/npl/sections/this/ biotest/index.html), 1998.

[9] P. J. Phillips and P. J. Rauss, "The Face Recognition Technology (FERET) Program", in Proc. of National Drug Control Policy, CTAC Intl. Tech. Symposium, Chicago, 1997.

[10] R. Cappelli, A. Erol, D. Maio and D. Maltoni, "Synthetic Fingerprint-image Generation", proceedings $15^{\text {th }}$ International Conference on Pattern Recognition (ICPR2000), Barcelona, September 2000.

[11] J.L. Wayman, "The Philippine AFIS Benchmark Test" in National Biometric Test Center Collected Works, 1997-2000, September, 2000 (available on-line at www.engr.sjsu.edu/biometrics/collected). 


\section{APPENDIX A}

\section{Algorithm Cetp on database $D B 1 \_A$}

Average enroll time: 0.81 seconds

Average match time: 0.89 seconds

\begin{tabular}{|c|c|c|c|c|}
\hline REJ $_{\text {ENROLL }}$ & NGRA & NIRA & REJ $_{\text {NGRA }}$ & REJ $_{\text {NIRA }}$ \\
\hline $0.00 \%(\mathrm{~F}: 0 \mathrm{~T}: 0 \mathrm{C}: 0)$ & 2800 & 4950 & $0.00 \%(\mathrm{~F}: 0 \mathrm{~T}: 0 \mathrm{C}: 0)$ & $0.00 \%(\mathrm{~F}: 0 \mathrm{~T}: 0 \mathrm{C}: 0)$ \\
\hline
\end{tabular}

\begin{tabular}{|c|c|c|c|}
\hline EER & EER* & ZeroFMR & ZeroFNMR \\
\hline $5.06 \%(4.30 \%-5.82 \%)$ & $5.06 \%(4.30 \%-5.82 \%)$ & $18.86 \%$ & $100.00 \%$ \\
\hline
\end{tabular}

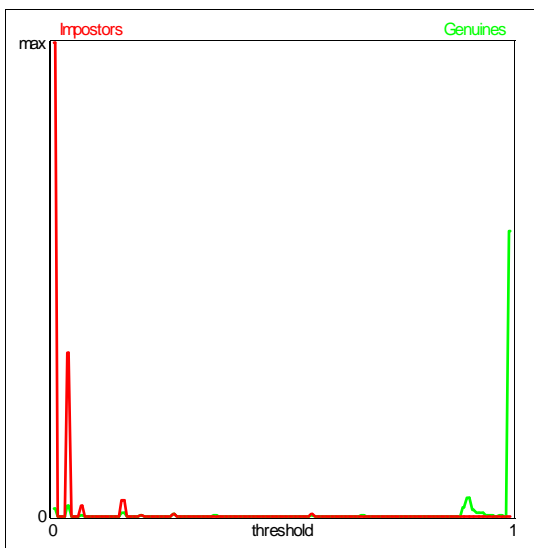

Score distributions

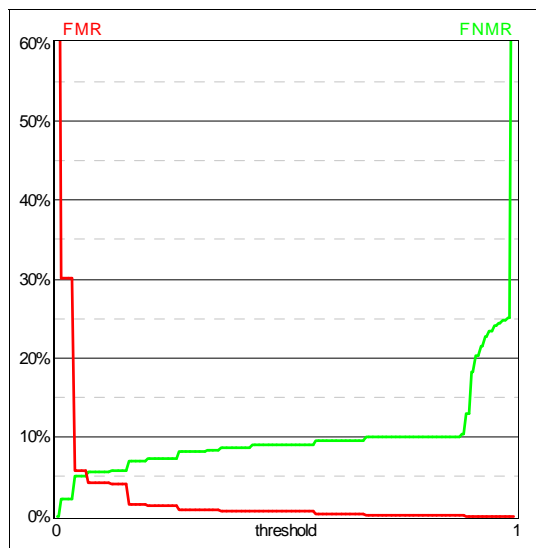

$\operatorname{FMR}(\mathrm{t})$ and $\operatorname{FNMR}(\mathrm{t})$

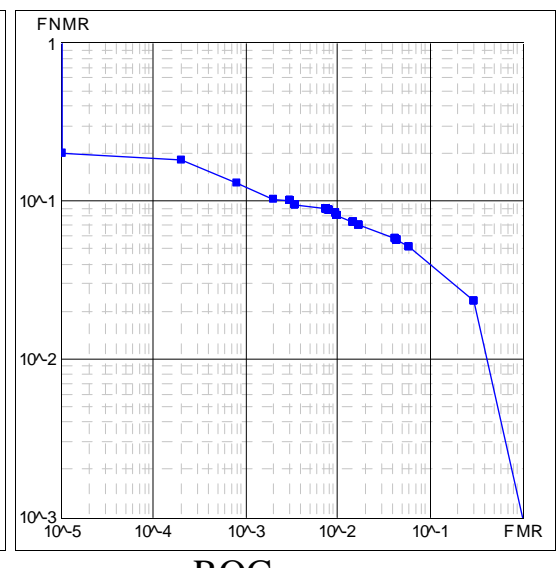

ROC curve

\section{Algorithm Cetp on database $D B 2 A$}

Average enroll time: 0.85 seconds

Average match time: 0.98 seconds

\begin{tabular}{|c|c|c|c|c|}
\hline REJ $_{\text {ENROLL }}$ & NGRA & NIRA & REJ $_{\text {NGRA }}$ & REJ $_{\text {NIRA }}$ \\
\hline $0.00 \%(\mathrm{~F}: 0 \mathrm{~T}: 0 \mathrm{C}: 0)$ & 2800 & 4950 & $0.25 \%(\mathrm{~F}: 0 \mathrm{~T}: 0 \mathrm{C}: 7)$ & $0.00 \%(\mathrm{~F}: 0 \mathrm{~T}: 0 \mathrm{C}: 0)$ \\
\hline
\end{tabular}

\begin{tabular}{|c|c|c|c|}
\hline EER & EER* & ZeroFMR & ZeroFNMR \\
\hline $4.63 \%(3.58 \%-5.68 \%)$ & $4.51 \%(3.58 \%-5.44 \%)$ & $11.71 \%$ & $100.00 \%$ \\
\hline
\end{tabular}

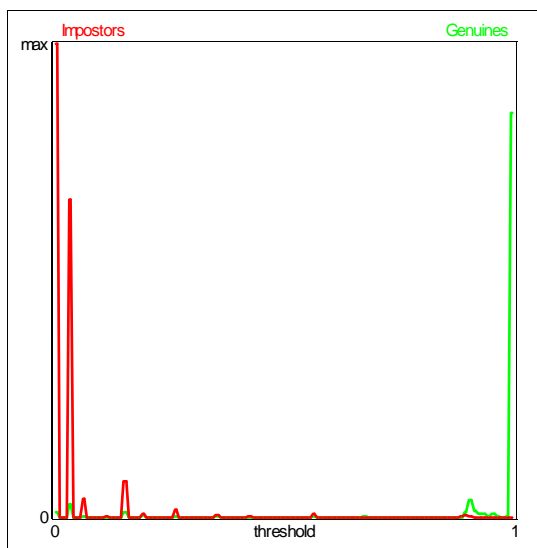

Score distributions

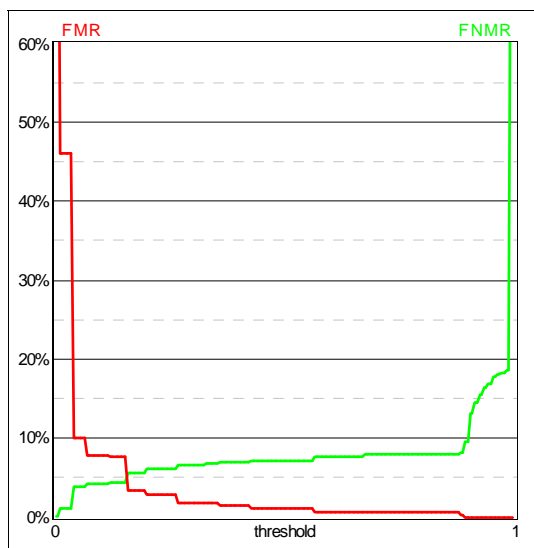

FMR(t) and FNMR(t)

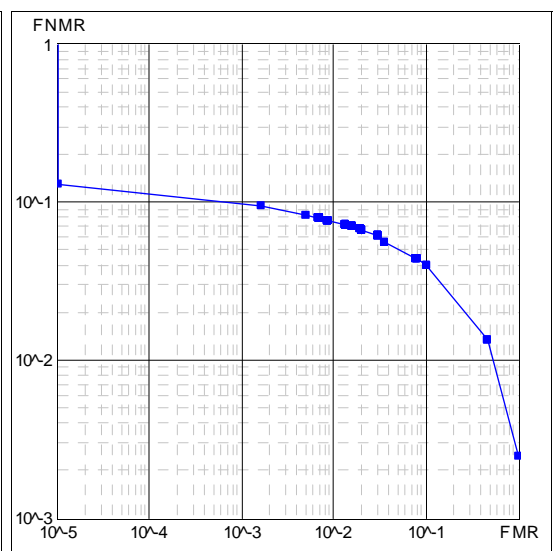

ROC curve 
Algorithm Cetp on database DB3_A

Average enroll time: 1.49 seconds Average match time: 1.66 seconds

\begin{tabular}{|c|c|c|c|c|}
\hline REJ $_{\text {ENROLL }}$ & NGRA & NIRA & REJ $_{\text {NGRA }}$ & REJ $_{\text {NIRA }}$ \\
\hline $0.00 \%(\mathrm{~F}: 0 \mathrm{~T}: 0 \mathrm{C}: 0)$ & 2800 & 4950 & $0.00 \%(\mathrm{~F}: 0 \mathrm{~T}: 0 \mathrm{C}: 0)$ & $0.00 \%(\mathrm{~F}: 0 \mathrm{~T}: 0 \mathrm{C}: 0)$ \\
\hline
\end{tabular}

\begin{tabular}{|c|c|c|c|}
\hline EER & EER* & ZeroFMR & ZeroFNMR \\
\hline $8.29 \%(6.55 \%-10.04 \%)$ & $8.29 \%(6.55 \%-10.04 \%)$ & $22.61 \%$ & $100.00 \%$ \\
\hline
\end{tabular}

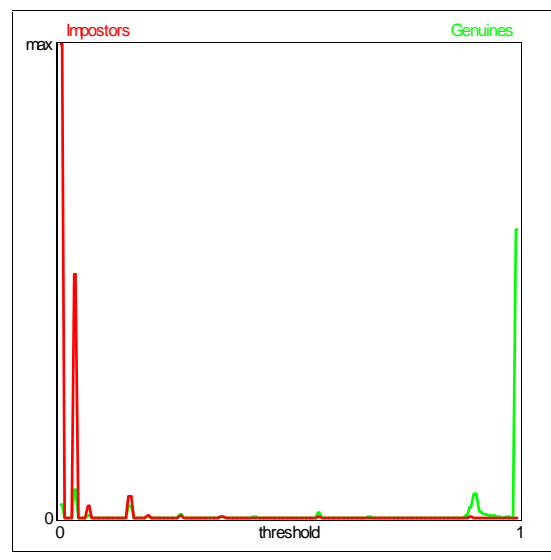

Score distributions

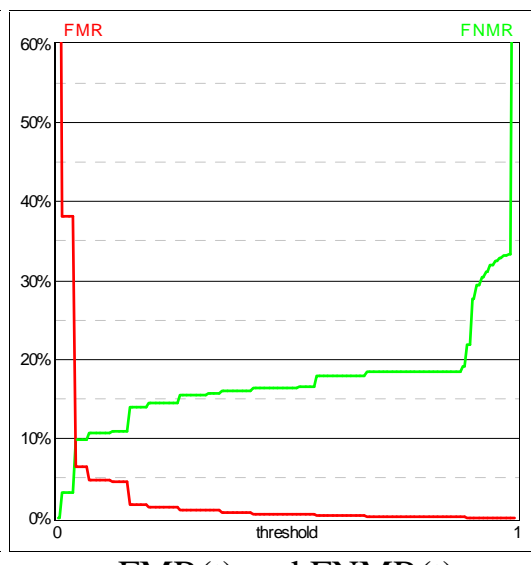

$\operatorname{FMR}(\mathrm{t})$ and $\operatorname{FNMR}(\mathrm{t})$

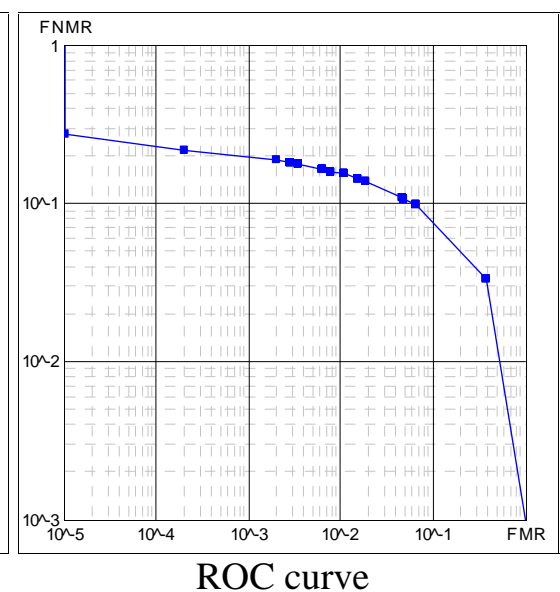

ROC curve

\section{Algorithm Cetp on database DB4_A}

Average enroll time: 0.65 seconds

Average match time: 0.72 seconds

\begin{tabular}{|c|c|c|c|c|}
\hline REJ $_{\text {ENROLL }}$ & NGRA & NIRA & $\mathbf{R E J}_{\text {NGRA }}$ & $\mathbf{R E J}_{\text {NIRA }}$ \\
\hline $0.00 \%(\mathrm{~F}: 0 \mathrm{~T}: 0 \mathrm{C}: 0)$ & 2800 & 4950 & $0.00 \%$ (F:0 T:0 C:0) & $0.00 \%$ (F:0 T:0 C:0) \\
\hline EER & \multicolumn{2}{|c|}{ EER* } & ZeroFMR & ZeroFNMR \\
\hline $7.29 \%(7.01 \%-7.57 \%)$ & \multicolumn{2}{|c|}{$7.29 \%(7.01 \%-7.57 \%)$} & $29.75 \%$ & $100.00 \%$ \\
\hline
\end{tabular}

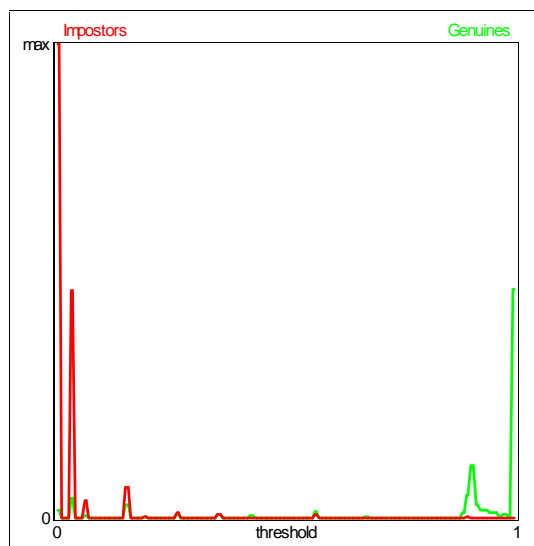

Score distributions

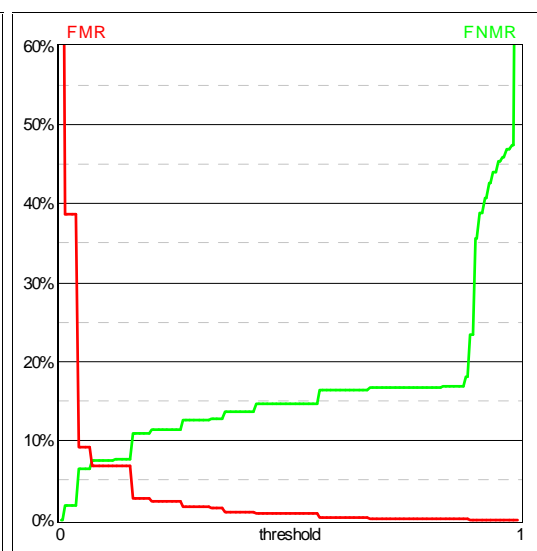

FMR(t) and $\operatorname{FNMR}(\mathrm{t})$

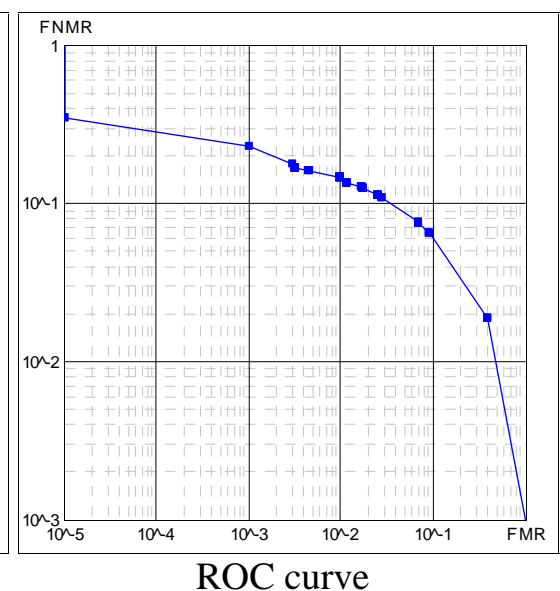




\section{Algorithm Cspn on database $D B 1 \_A$}

Average enroll time: 0.17 seconds

Average match time: 0.17 seconds

\begin{tabular}{|c|c|c|c|c|}
\hline REJ $_{\text {ENROLL }}$ & NGRA & NIRA & REJ $_{\text {NGRA }}$ & REJ $_{\text {NIRA }}$ \\
\hline $0.00 \%(\mathrm{~F}: 0 \mathrm{~T}: 0 \mathrm{C}: 0)$ & 2800 & 4950 & $0.00 \%(\mathrm{~F}: 0 \mathrm{~T}: 0 \mathrm{C}: 0)$ & $0.00 \%(\mathrm{~F}: 0 \mathrm{~T}: 0 \mathrm{C}: 0)$ \\
\hline
\end{tabular}

\begin{tabular}{|c|c|c|c|}
\hline EER & EER* & ZeroFMR & ZeroFNMR \\
\hline $7.60 \%(7.60 \%-7.61 \%)$ & $7.60 \%(7.60 \%-7.61 \%)$ & $22.46 \%$ & $100.00 \%$ \\
\hline
\end{tabular}

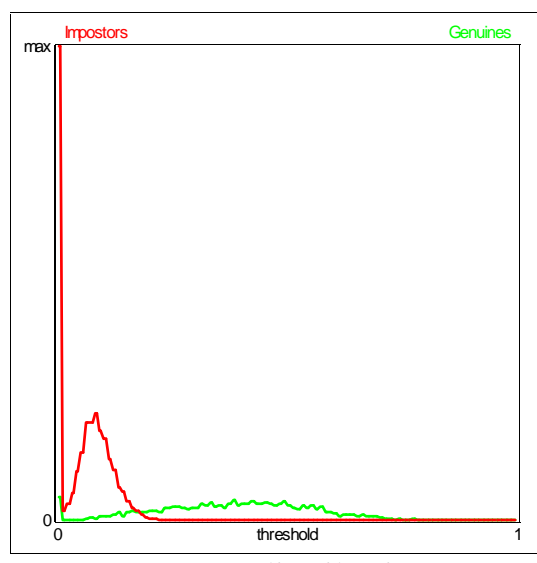

Score distributions

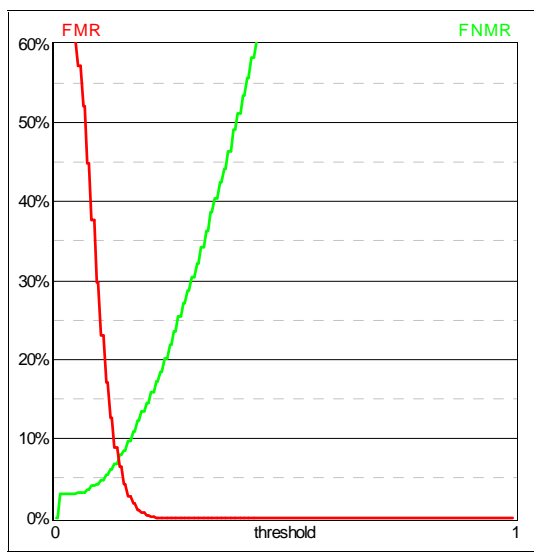

FMR (t) and FNMR(t)

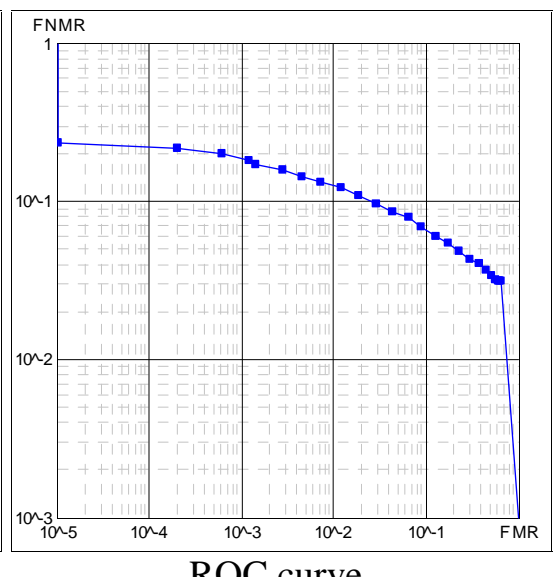

ROC curve

\section{Algorithm Cspn on database $D B 2$ A}

Average enroll time: 0.17 seconds

Average match time: 0.17 seconds

\begin{tabular}{|c|c|c|c|c|}
\hline REJ $_{\text {ENROLL }}$ & NGRA & NIRA & REJ $_{\text {NGRA }}$ & REJ $_{\text {NIRA }}$ \\
\hline $0.00 \%(\mathrm{~F}: 0 \mathrm{~T}: 0 \mathrm{C}: 0)$ & 2800 & 4950 & $0.00 \%(\mathrm{~F}: 0 \mathrm{~T}: 0 \mathrm{C}: 0)$ & $0.00 \%(\mathrm{~F}: 0 \mathrm{~T}: 0 \mathrm{C}: 0)$ \\
\hline
\end{tabular}

\begin{tabular}{|c|c|c|c|}
\hline EER & EER* & ZeroFMR & ZeroFNMR \\
\hline $2.75 \%$ & $2.75 \%$ & $10.29 \%$ & $100.00 \%$ \\
\hline
\end{tabular}

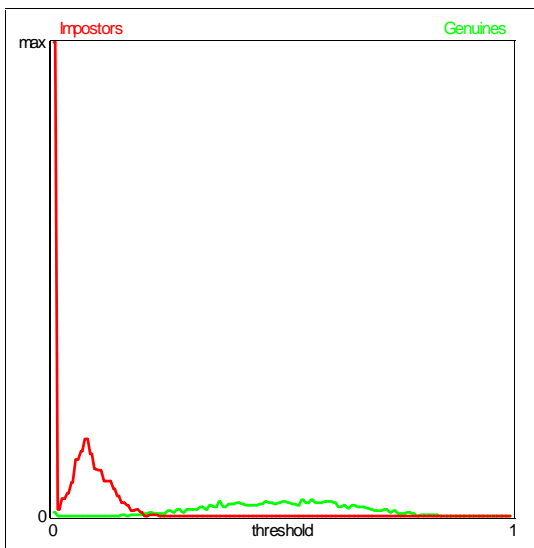

Score distributions

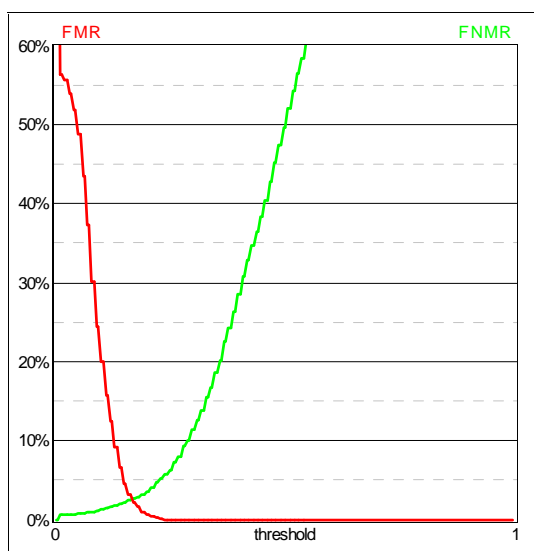

FMR $(t)$ and $F N M R(t)$

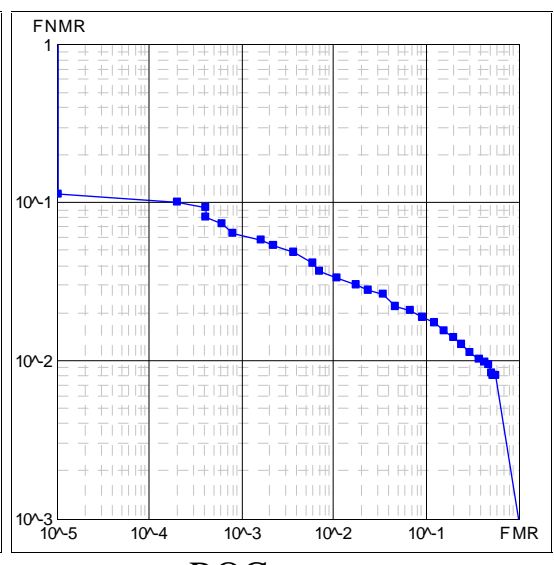

ROC curve 


\section{Algorithm Cspn on database $D B 3 \_A$}

Average enroll time: 0.35 seconds Average match time: 0.36 seconds

\begin{tabular}{|c|c|c|c|c|}
\hline REJ $_{\text {ENROLL }}$ & NGRA & NIRA & REJ $_{\text {NGRA }}$ & $\mathbf{R E J}_{\text {NIRA }}$ \\
\hline $0.57 \%(\mathrm{~F}: 0 \mathrm{~T}: 0 \mathrm{C}: 4)$ & 2777 & 4945 & $0.07 \%(\mathrm{~F}: 0 \mathrm{~T}: 0 \mathrm{C}: 2)$ & $1.90 \%(\mathrm{~F}: 0 \mathrm{~T}: 0 \mathrm{C}: 94)$ \\
\hline
\end{tabular}

\begin{tabular}{|c|c|c|c|}
\hline EER & EER* & ZeroFMR & ZeroFNMR \\
\hline $5.36 \%$ & $5.33 \%(5.32 \%-5.33 \%)$ & $21.07 \%$ & $100.00 \%$ \\
\hline
\end{tabular}

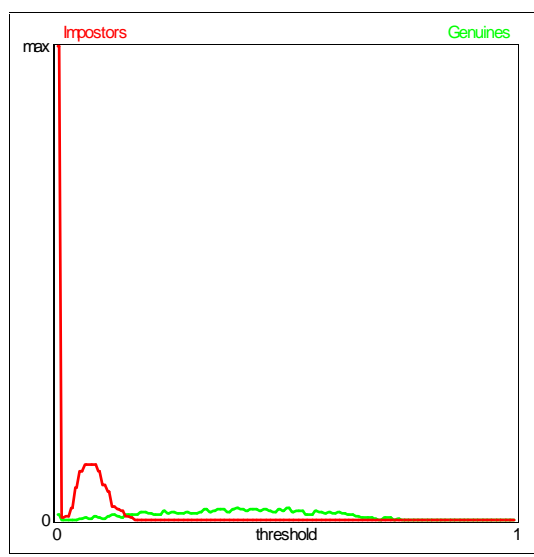

Score distributions

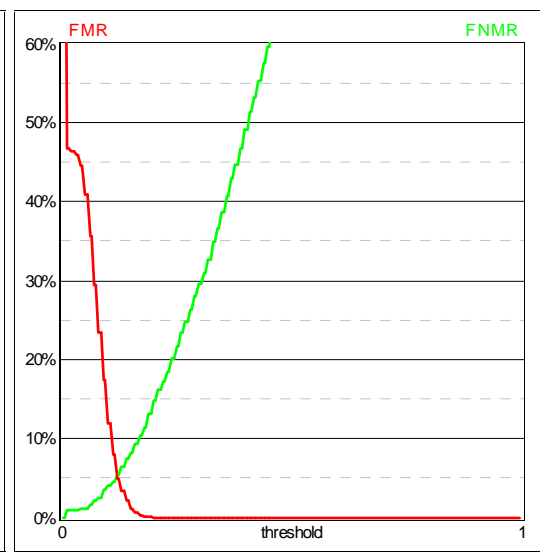

FMR(t) and FNMR(t)

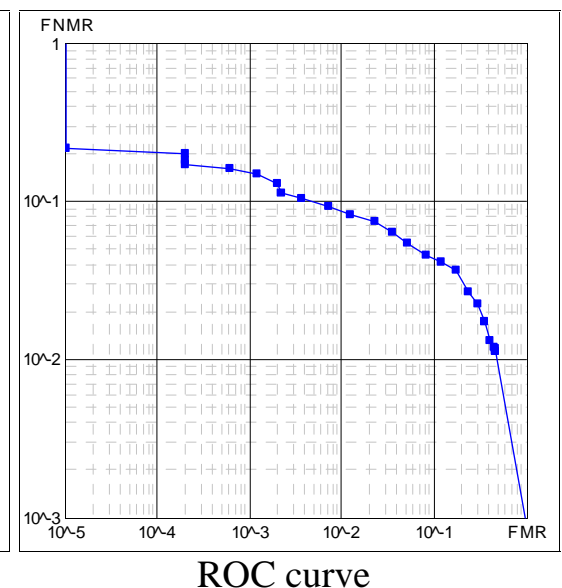

\section{Algorithm Cspn on database $D B 4 \_A$}

Average enroll time: 0.11 seconds

Average match time: 0.11 seconds

\begin{tabular}{|c|c|c|c|c|}
\hline REJ $_{\text {ENROLL }}$ & NGRA & NIRA & $\mathbf{R E J}_{\text {NGRA }}$ & $\mathbf{R E J}_{\text {NIRA }}$ \\
\hline $0.00 \%(\mathrm{~F}: 0 \mathrm{~T}: 0 \mathrm{C}: 0)$ & 2800 & 4950 & $0.00 \%$ (F:0 T:0 C:0) & $0.00 \%$ (F:0 T:0 C:0) \\
\hline EER & \multicolumn{2}{|c|}{ EER* } & ZeroFMR & ZeroFNMR \\
\hline $5.04 \%(5.04 \%-5.05 \%)$ & \multicolumn{2}{|c|}{$5.04 \%(5.04 \%-5.05 \%)$} & $15.54 \%$ & $100.00 \%$ \\
\hline
\end{tabular}

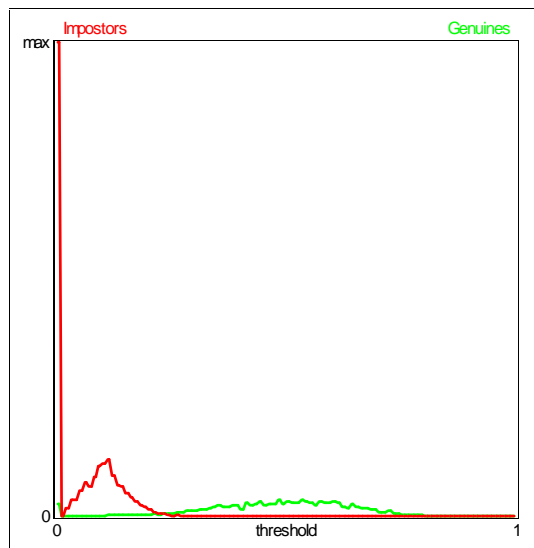

Score distributions

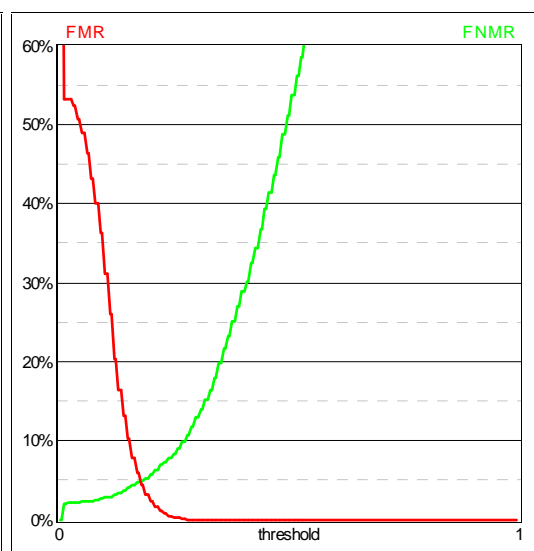

FMR(t) and $\operatorname{FNMR}(\mathrm{t})$

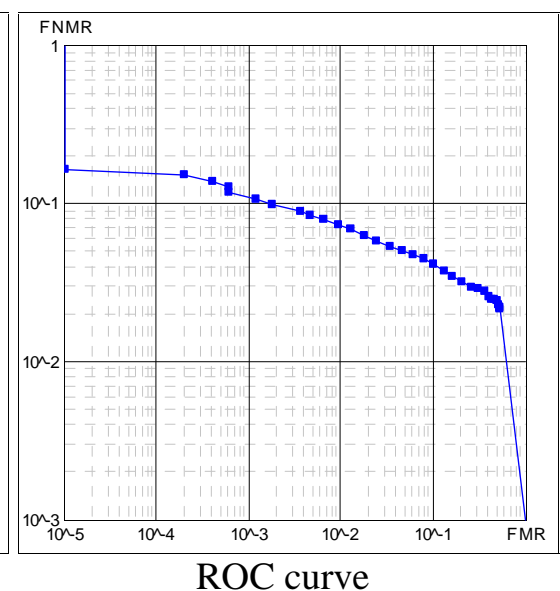




\section{Algorithm $C$ wai on database $D B 1 \_A$}

Average enroll time: 0.22 seconds

Average match time: 0.32 seconds

\begin{tabular}{|c|c|c|c|c|}
\hline REJ $_{\text {ENROLL }}$ & NGRA & NIRA & REJ $_{\text {NGRA }}$ & REJ $_{\text {NIRA }}$ \\
\hline $3.71 \%(\mathrm{~F}: 26 \mathrm{~T}: 0 \mathrm{C}: 0)$ & 2717 & 4944 & $3.97 \%(\mathrm{~F}: 108 \mathrm{~T}: 0 \mathrm{C}: 0)$ & $3.86 \%(\mathrm{~F}: 191 \mathrm{~T}: 0 \mathrm{C}: 0)$ \\
\hline
\end{tabular}

\begin{tabular}{|c|c|c|c|}
\hline EER & EER* & ZeroFMR & ZeroFNMR \\
\hline $7.06 \%(6.61 \%-7.51 \%)$ & $4.27 \%(4.10 \%-4.45 \%)$ & $23.15 \%$ & $100.00 \%$ \\
\hline
\end{tabular}

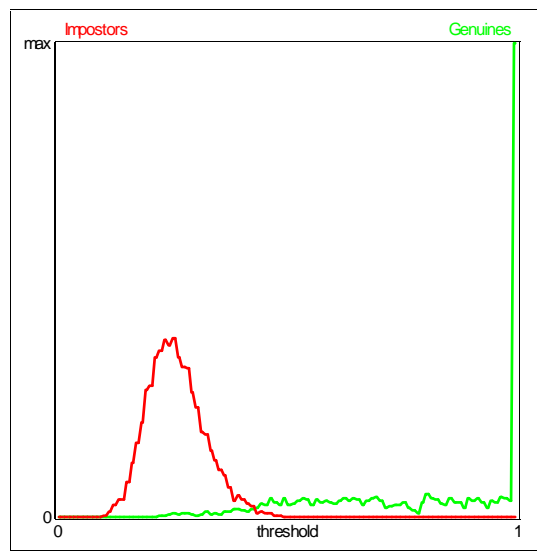

Score distributions

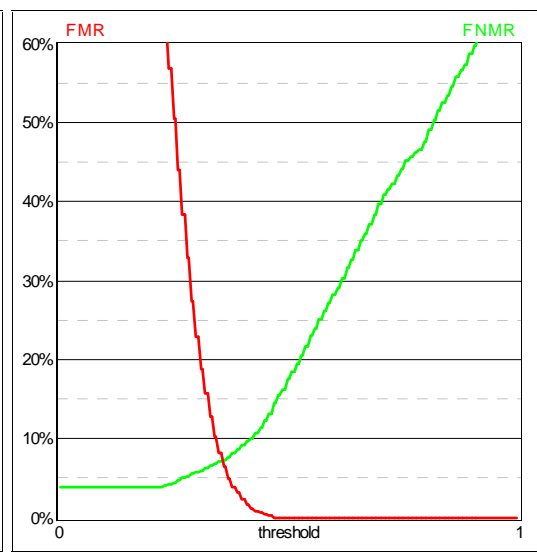

FMR (t) and FNMR(t)

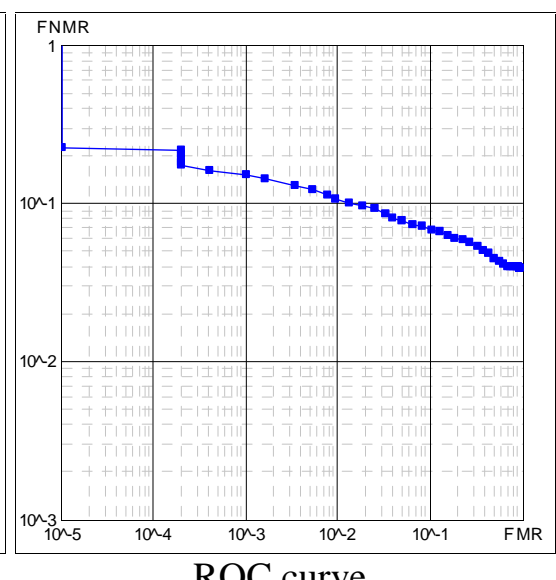

ROC curve

\section{Algorithm $C$ wai on database $D B 2$ A}

Average enroll time: 0.23 seconds

Average match time: 0.30 seconds

\begin{tabular}{|c|c|c|c|c|}
\hline REJ $_{\text {ENROLL }}$ & NGRA & NIRA & REJ $_{\text {NGRA }}$ & REJ $_{\text {NIRA }}$ \\
\hline $1.29 \%(\mathrm{~F}: 9 \mathrm{~T}: 0 \mathrm{C}: 0)$ & 2768 & 4916 & $1.23 \%(\mathrm{~F}: 34 \mathrm{~T}: 0 \mathrm{C}: 0)$ & $1.32 \%(\mathrm{~F}: 65 \mathrm{~T}: 0 \mathrm{C}: 0)$ \\
\hline
\end{tabular}

\begin{tabular}{|c|c|c|c|}
\hline EER & EER* & ZeroFMR & ZeroFNMR \\
\hline $3.01 \%(2.66 \%-3.36 \%)$ & $2.16 \%(2.02 \%-2.30 \%)$ & $8.74 \%$ & $100.00 \%$ \\
\hline
\end{tabular}

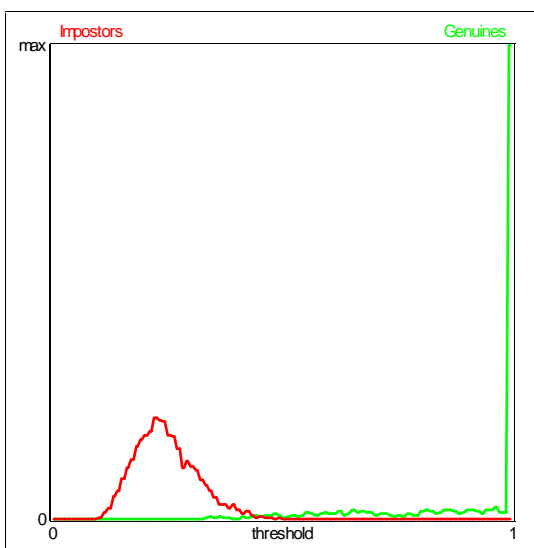

Score distributions

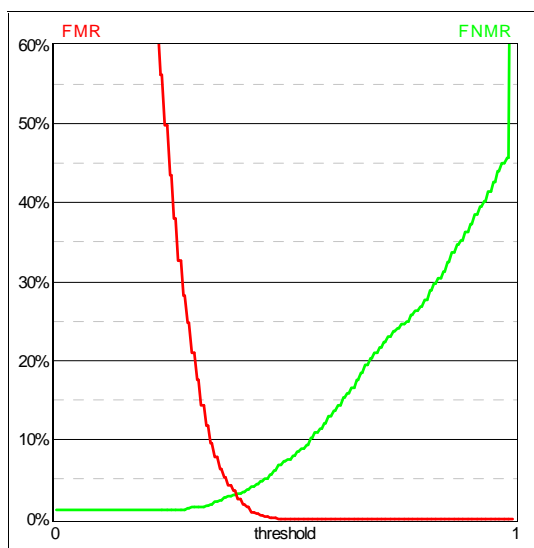

$\operatorname{FMR}(\mathrm{t})$ and $\operatorname{FNMR}(\mathrm{t})$

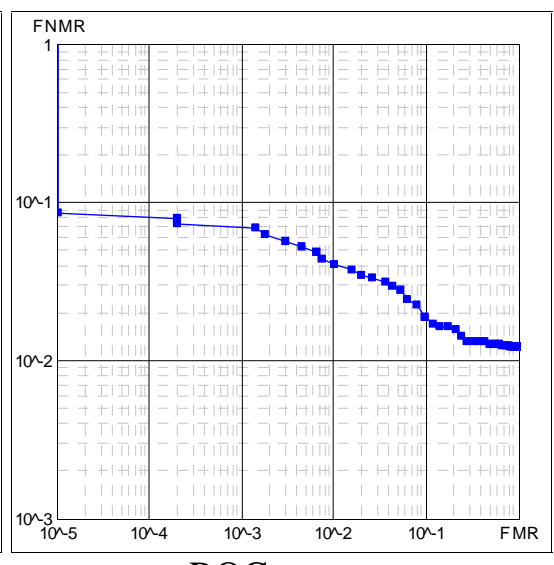

ROC curve 


\section{Algorithm $C$ wai on database $D B 3 \_A$}

Average enroll time: 0.46 seconds Average match time: 0.57 seconds

\begin{tabular}{|c|c|c|c|c|}
\hline REJ $_{\text {ENROLL }}$ & NGRA & NIRA & REJ $\mathbf{J}_{\text {NGRA }}$ & $\mathbf{R E J}_{\text {NIRA }}$ \\
\hline $12.86 \%$ (F:90 T:0 C:0) & 2475 & 4252 & 8.16\% (F:202 T:0 C:0) & $7.90 \%(\mathrm{~F}: 336 \mathrm{~T}: 0 \mathrm{C}: 0)$ \\
\hline EER & \multicolumn{2}{|c|}{ EER* } & ZeroFMR & ZeroFNMR \\
\hline $11.94 \%(10.87 \%-13.01 \%)$ & \multicolumn{2}{|c|}{$5.90 \%(5.03 \%-6.78 \%)$} & $23.43 \%$ & $100.00 \%$ \\
\hline
\end{tabular}

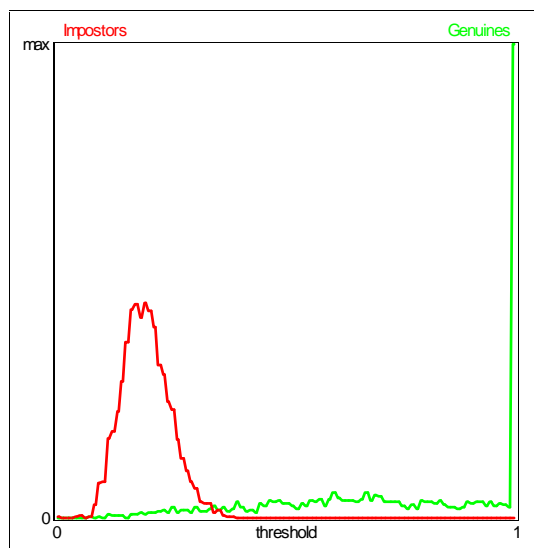

Score distributions

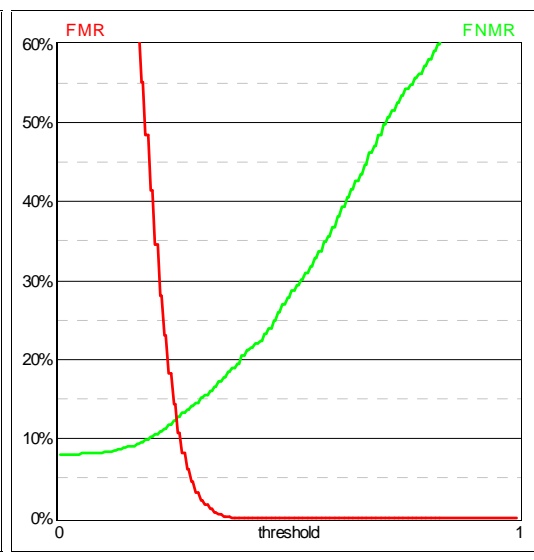

FMR(t) and FNMR(t)

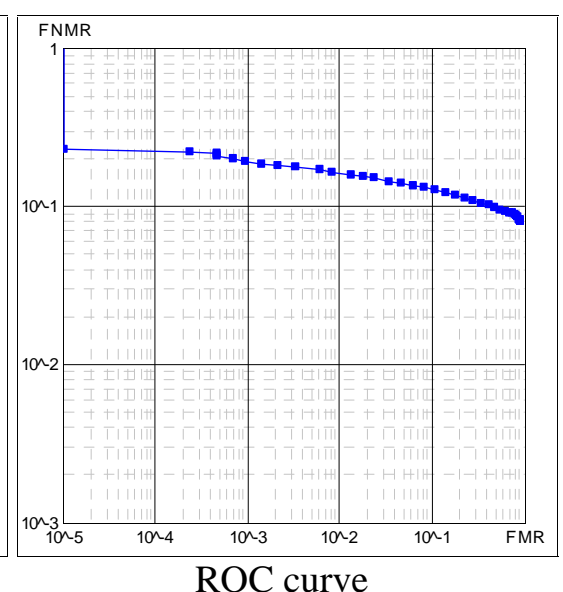

ROC curve

\section{Algorithm $C$ wai on database $D B 4$ A}

Average enroll time: 0.16 seconds

Average match time: 0.20 seconds

\begin{tabular}{|c|c|c|c|c|}
\hline REJ $_{\text {ENROLL }}$ & NGRA & NIRA & $\mathbf{R E J}_{\text {NGRA }}$ & $\mathbf{R E J}_{\text {NIRA }}$ \\
\hline $0.00 \%(\mathrm{~F}: 0 \mathrm{~T}: 0 \mathrm{C}: 0)$ & 2800 & 4950 & $0.00 \%$ (F:0 T:0 C:0) & $0.00 \%$ (F:0 T:0 C:0) \\
\hline EER & \multicolumn{2}{|c|}{ EER* } & ZeroFMR & ZeroFNMR \\
\hline $6.30 \%(5.74 \%-6.86 \%)$ & \multicolumn{2}{|c|}{$6.30 \%(5.74 \%-6.86 \%)$} & $42.18 \%$ & $78.34 \%$ \\
\hline
\end{tabular}

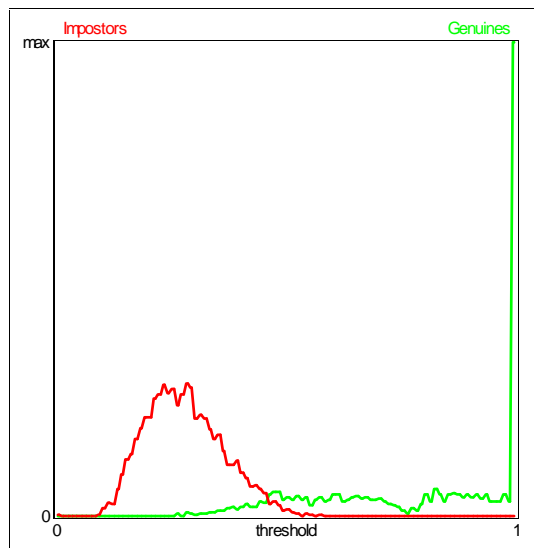

Score distributions

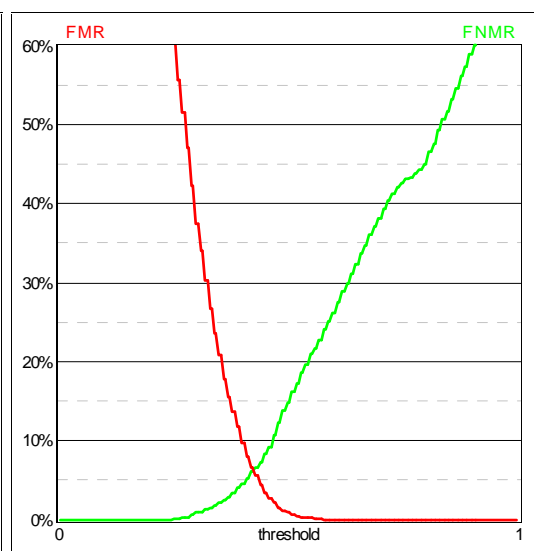

FMR(t) and FNMR(t)

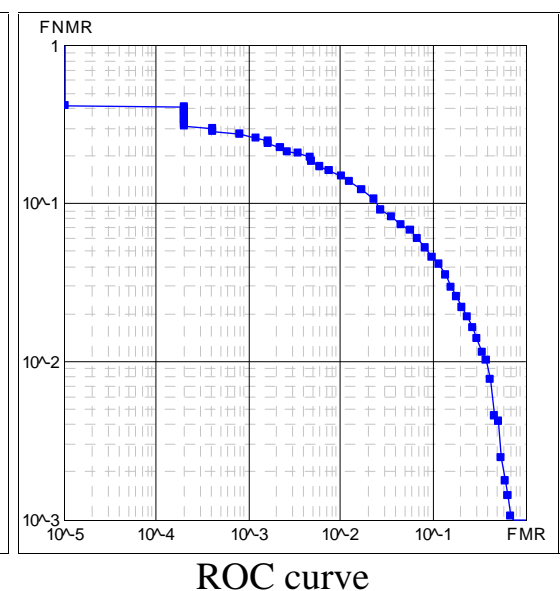




\section{Algorithm Diti on database $D B 1 A$}

Average enroll time: 0.65 seconds

Average match time: 0.72 seconds

\begin{tabular}{|c|c|c|c|c|}
\hline REJ $_{\text {ENROLL }}$ & NGRA & NIRA & REJ $_{\text {NGRA }}$ & REJ $_{\text {NIRA }}$ \\
\hline $0.00 \%(\mathrm{~F}: 0 \mathrm{~T}: 0 \mathrm{C}: 0)$ & 2800 & 4950 & $0.00 \%(\mathrm{~F}: 0 \mathrm{~T}: 0 \mathrm{C}: 0)$ & $0.00 \%(\mathrm{~F}: 0 \mathrm{~T}: 0 \mathrm{C}: 0)$ \\
\hline
\end{tabular}

\begin{tabular}{|c|c|c|c|}
\hline EER & EER* & ZeroFMR & ZeroFNMR \\
\hline $23.63 \%(23.62 \%-23.64 \%)$ & $23.63 \%(23.62 \%-23.64 \%)$ & $50.54 \%$ & $100.00 \%$ \\
\hline
\end{tabular}

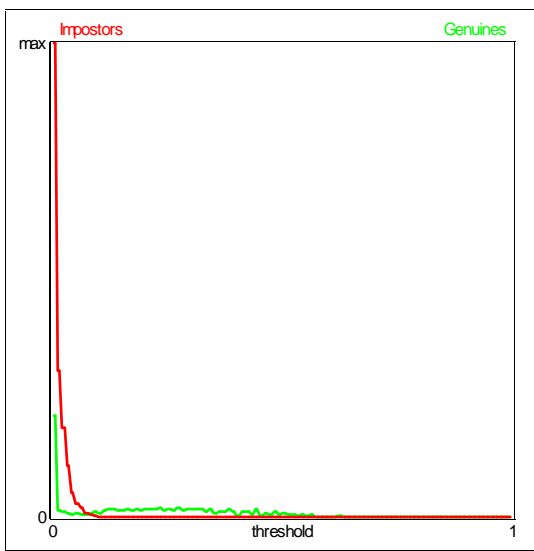

Score distributions

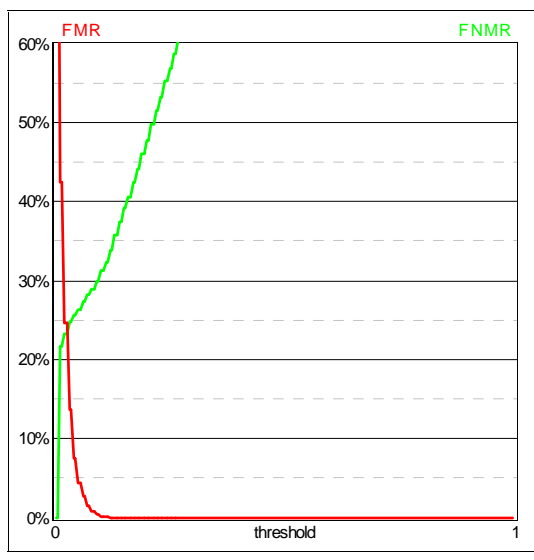

FMR(t) and FNMR(t)

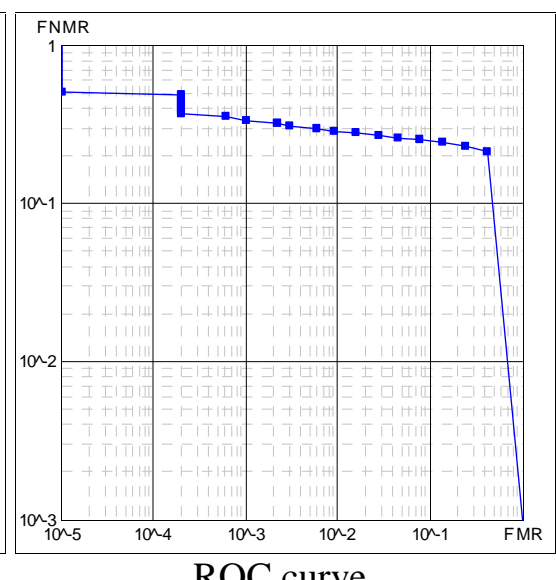

ROC curve

\section{Algorithm Diti on database $D B 2$ A}

Average enroll time: 1.21 seconds

Average match time: 1.28 seconds

\begin{tabular}{|c|c|c|c|c|}
\hline REJ $_{\text {ENROLL }}$ & NGRA & NIRA & REJ $_{\text {NGRA }}$ & REJ $_{\text {NIRA }}$ \\
\hline $0.00 \%(\mathrm{~F}: 0 \mathrm{~T}: 0 \mathrm{C}: 0)$ & 2800 & 4950 & $0.00 \%(\mathrm{~F}: 0 \mathrm{~T}: 0 \mathrm{C}: 0)$ & $0.00 \%(\mathrm{~F}: 0 \mathrm{~T}: 0 \mathrm{C}: 0)$ \\
\hline
\end{tabular}

\begin{tabular}{|c|c|c|c|}
\hline EER & EER $^{*}$ & ZeroFMR & ZeroFNMR \\
\hline $13.83 \%(13.80 \%-13.86 \%)$ & $13.83 \%(13.80 \%-13.86 \%)$ & $37.43 \%$ & $100.00 \%$ \\
\hline
\end{tabular}

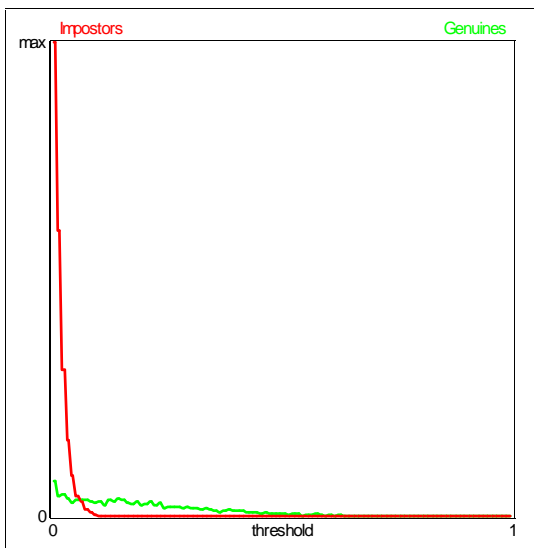

Score distributions

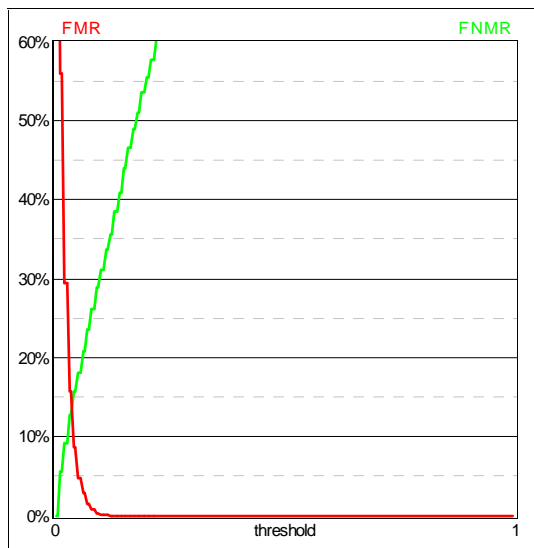

FMR $(t)$ and $F N M R(t)$

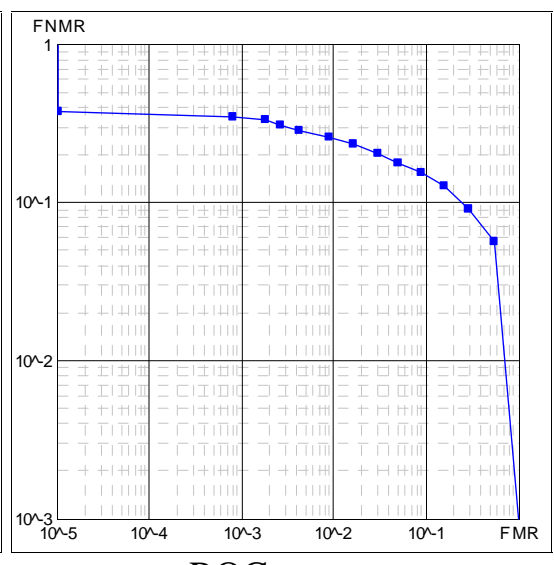

ROC curve 


\section{Algorithm Diti on database $D B 3 \_A$}

Average enroll time: 2.59 seconds Average match time: 2.67 seconds

\begin{tabular}{|c|c|c|c|c|}
\hline REJ $_{\text {ENROLL }}$ & NGRA & NIRA & REJ $_{\text {NGRA }}$ & REJ $\mathbf{J}_{\text {NIRA }}$ \\
\hline $0.00 \%(\mathrm{~F}: 0 \mathrm{~T}: 0 \mathrm{C}: 0)$ & 2800 & 4950 & $0.00 \%(\mathrm{~F}: 0 \mathrm{~T}: 0 \mathrm{C}: 0)$ & $0.00 \%$ (F:0 T:0 C:0) \\
\hline
\end{tabular}

\begin{tabular}{|c|c|c|c|}
\hline EER & EER* & ZeroFMR & ZeroFNMR \\
\hline $22.63 \%(22.61 \%-22.65 \%)$ & $22.63 \%(22.61 \%-22.65 \%)$ & $65.54 \%$ & $100.00 \%$ \\
\hline
\end{tabular}

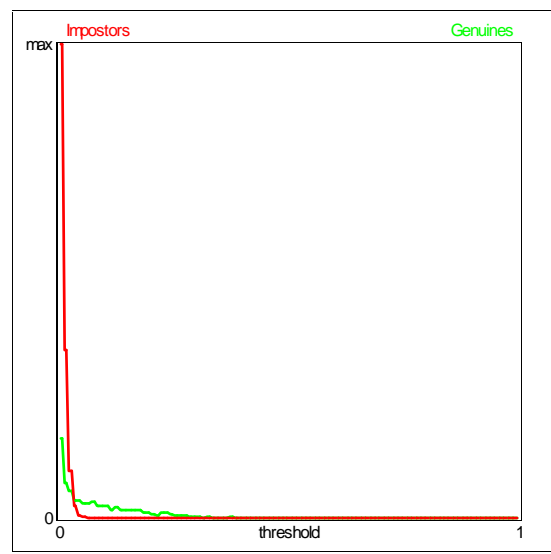

Score distributions

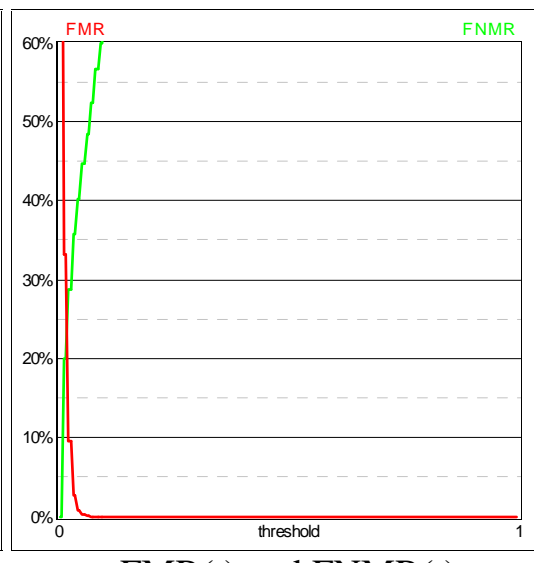

FMR(t) and FNMR(t)

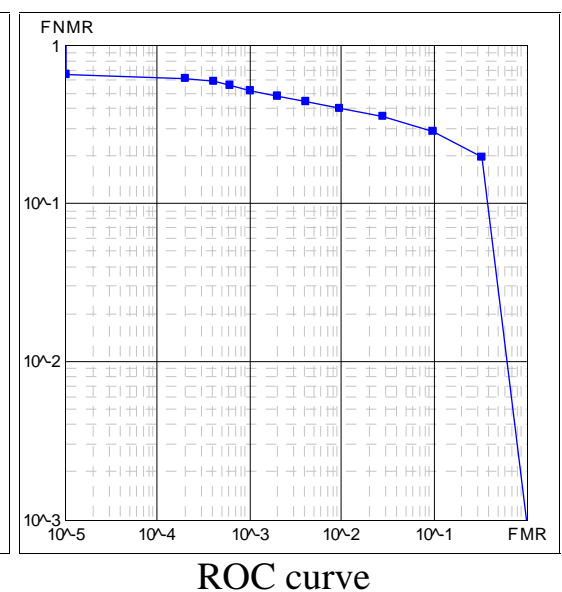

\section{Algorithm Diti on database $D B 4$ A}

Average enroll time: 0.52 seconds

Average match time: 0.60 seconds

\begin{tabular}{|c|c|c|c|c|}
\hline REJ $_{\text {ENROLL }}$ & NGRA & NIRA & REJ $_{\text {NGRA }}$ & REJ $_{\text {NIRA }}$ \\
\hline $0.00 \%(\mathrm{~F}: 0 \mathrm{~T}: 0 \mathrm{C}: 0)$ & 2800 & 4950 & $0.00 \%(\mathrm{~F}: 0 \mathrm{~T}: 0 \mathrm{C}: 0)$ & $0.00 \%(\mathrm{~F}: 0 \mathrm{~T}: 0 \mathrm{C}: 0)$ \\
\hline
\end{tabular}

\begin{tabular}{|c|c|c|c|}
\hline EER & EER* & ZeroFMR & ZeroFNMR \\
\hline $23.80 \%(23.74 \%-23.86 \%)$ & $23.80 \%(23.74 \%-23.86 \%)$ & $75.36 \%$ & $100.00 \%$ \\
\hline
\end{tabular}

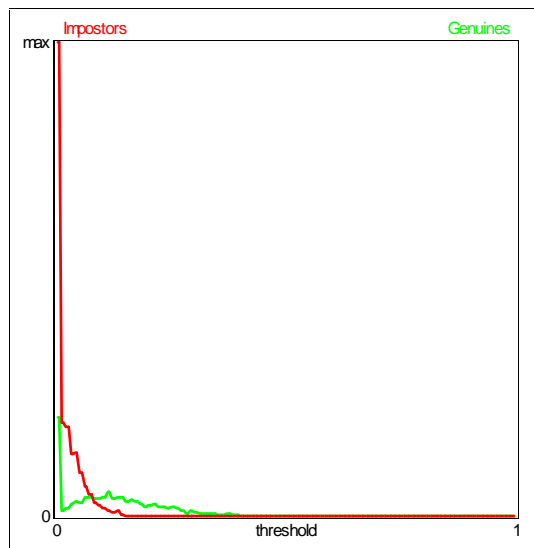

Score distributions

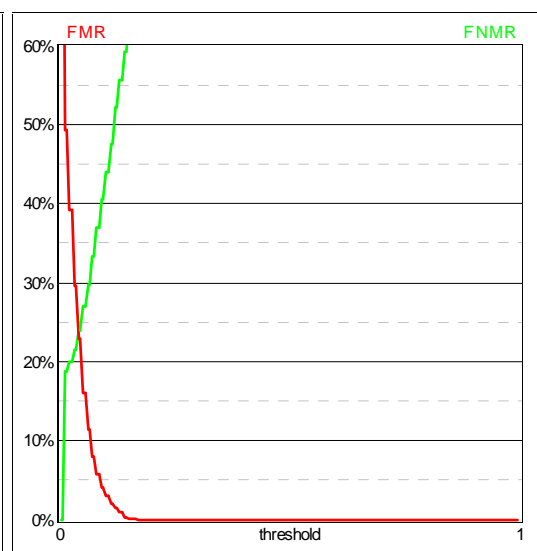

FMR(t) and FNMR(t)

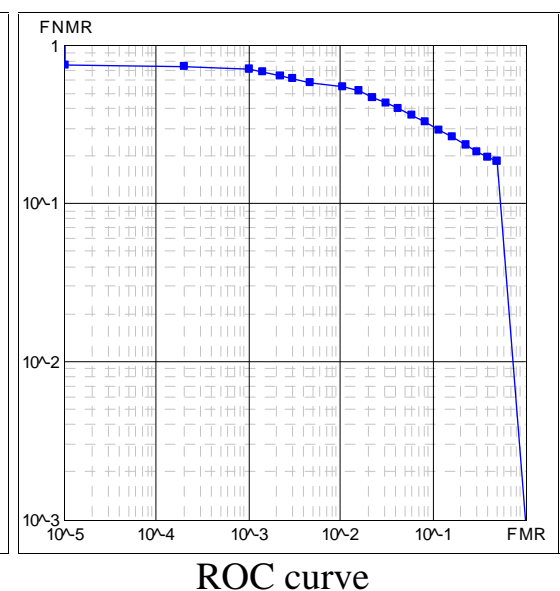




\section{Algorithm Fpin on database $D B 1 \_A$}

Average enroll time: 0.83 seconds

Average match time: 0.87 seconds

\begin{tabular}{|c|c|c|c|c|}
\hline REJ $_{\text {ENROLL }}$ & NGRA & NIRA & REJ $_{\text {NGRA }}$ & REJ $_{\text {NIRA }}$ \\
\hline $0.00 \%(\mathrm{~F}: 0 \mathrm{~T}: 0 \mathrm{C}: 0)$ & 2800 & 4950 & $0.00 \%(\mathrm{~F}: 0 \mathrm{~T}: 0 \mathrm{C}: 0)$ & $0.00 \%(\mathrm{~F}: 0 \mathrm{~T}: 0 \mathrm{C}: 0)$ \\
\hline
\end{tabular}

\begin{tabular}{|c|c|c|c|}
\hline EER & EER* & ZeroFMR & ZeroFNMR \\
\hline $13.46 \%$ & $13.46 \%$ & $96.07 \%$ & $100.00 \%$ \\
\hline
\end{tabular}

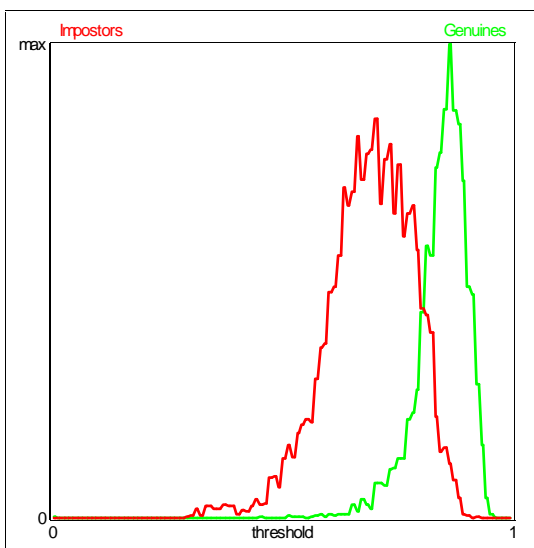

Score distributions

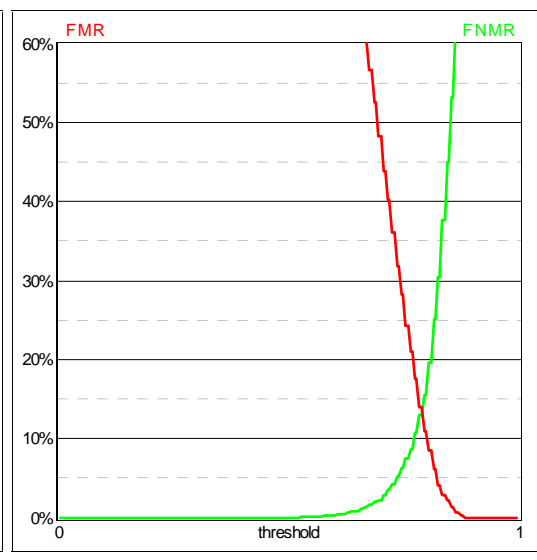

FMR (t) and FNMR(t)

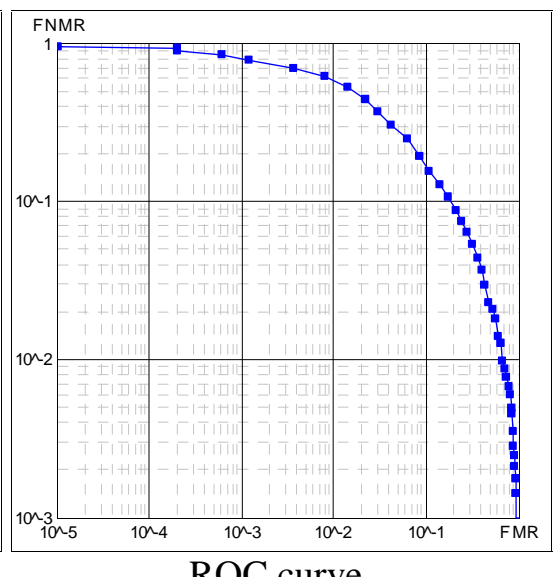

ROC curve

\section{Algorithm Fpin on database $D B 2 \_A$}

Average enroll time: 1.16 seconds

Average match time: 1.24 seconds

\begin{tabular}{|c|c|c|c|c|}
\hline REJ $_{\text {ENROLL }}$ & NGRA & NIRA & REJ $_{\text {NGRA }}$ & REJ $_{\text {NIRA }}$ \\
\hline $0.00 \%(\mathrm{~F}: 0 \mathrm{~T}: 0 \mathrm{C}: 0)$ & 2800 & 4950 & $0.00 \%(\mathrm{~F}: 0 \mathrm{~T}: 0 \mathrm{C}: 0)$ & $0.00 \%(\mathrm{~F}: 0 \mathrm{~T}: 0 \mathrm{C}: 0)$ \\
\hline
\end{tabular}

\begin{tabular}{|c|c|c|c|}
\hline EER & EER $^{*}$ & ZeroFMR & ZeroFNMR \\
\hline $11.14 \%(11.13 \%-11.14 \%)$ & $11.14 \%(11.13 \%-11.14 \%)$ & $95.61 \%$ & $99.45 \%$ \\
\hline
\end{tabular}

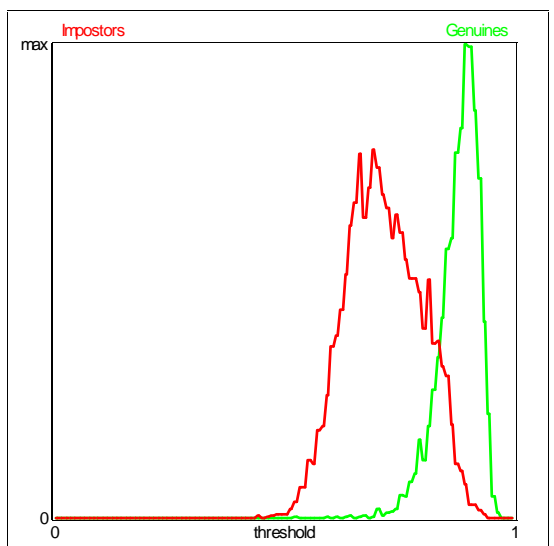

Score distributions

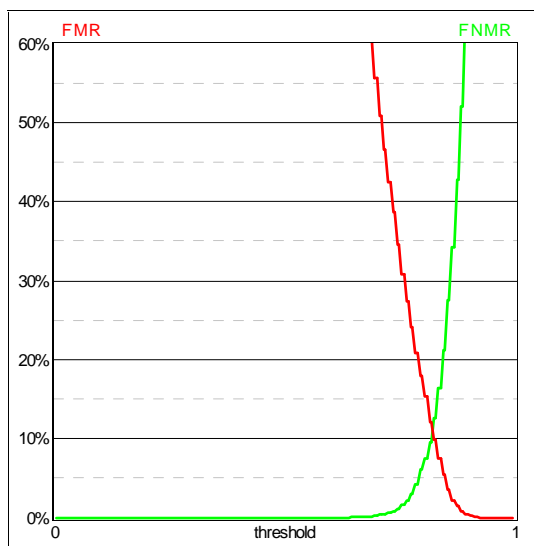

FMR $(t)$ and $F N M R(t)$

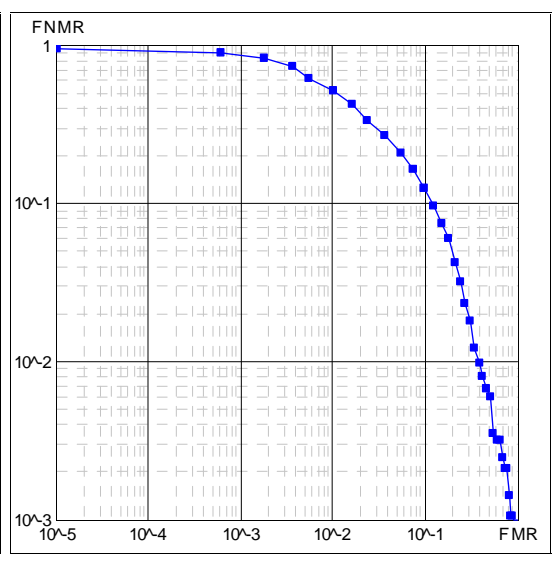

ROC curve 


\section{Algorithm Fpin on database $D B 3 \_A$}

Average enroll time: 2.13 seconds Average match time: 2.19 seconds

\begin{tabular}{|c|c|c|c|c|}
\hline REJ $_{\text {ENROLL }}$ & NGRA & NIRA & $\mathbf{R E J}_{\text {NGRA }}$ & $\mathbf{R E J}_{\text {NIRA }}$ \\
\hline $0.00 \%(\mathrm{~F}: 0 \mathrm{~T}: 0 \mathrm{C}: 0)$ & 2800 & 4950 & 0.00\% (F:0 T:0 C:0) & 0.00\% (F:0 T:0 C:0) \\
\hline EER & \multicolumn{2}{|c|}{ EER* } & ZeroFMR & ZeroFNMR \\
\hline $23.18 \%$ & \multicolumn{2}{|c|}{$23.18 \%$} & $98.61 \%$ & $100.00 \%$ \\
\hline
\end{tabular}

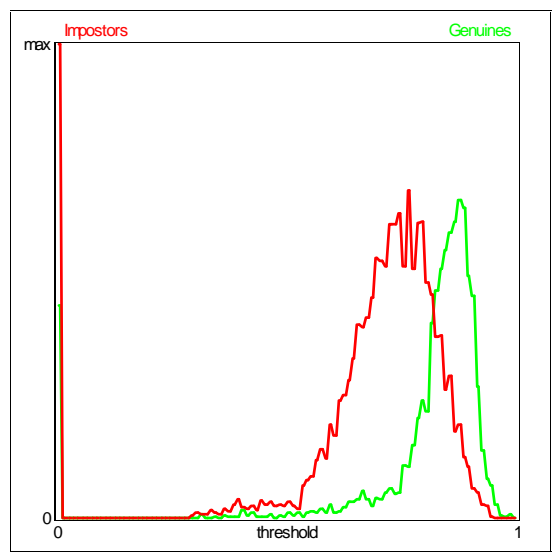

Score distributions

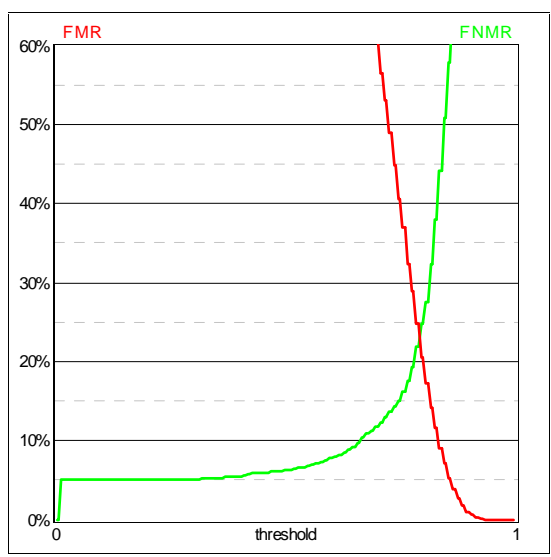

FMR(t) and FNMR(t)

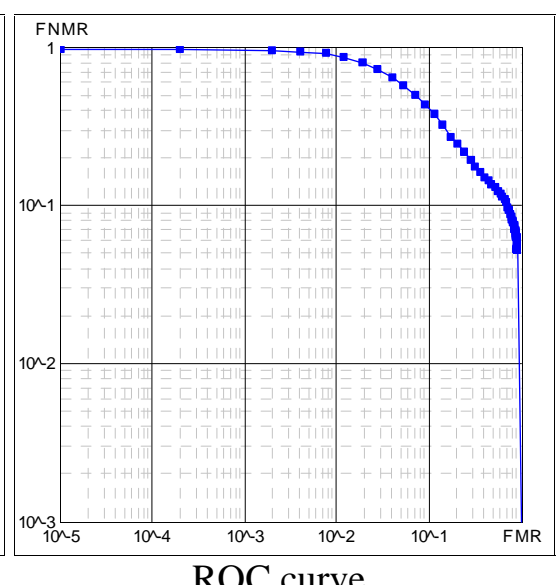

ROC curve

\section{Algorithm Fpin on database $D B 4 \_A$}

Average enroll time: 0.77 seconds

Average match time: 0.80 seconds

\begin{tabular}{|c|c|c|c|c|}
\hline REJ $_{\text {ENROLL }}$ & NGRA & NIRA & REJ $_{\text {NGRA }}$ & $\mathbf{R E J}_{\text {NIRA }}$ \\
\hline $0.00 \%(\mathrm{~F}: 0 \mathrm{~T}: 0 \mathrm{C}: 0)$ & 2800 & 4950 & $0.00 \%(\mathrm{~F}: 0 \mathrm{~T}: 0 \mathrm{C}: 0)$ & $0.00 \%(\mathrm{~F}: 0 \mathrm{~T}: 0 \mathrm{C}: 0)$ \\
\hline EER & \multicolumn{2}{|c|}{ EER* } & ZeroFMR & ZeroFNMR \\
\hline $16.00 \%$ & \multicolumn{2}{|c|}{$16.00 \%$} & $97.89 \%$ & $80.02 \%$ \\
\hline
\end{tabular}

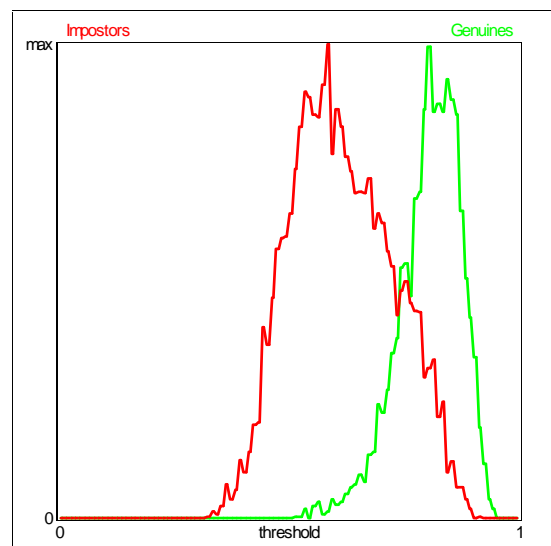

Score distributions

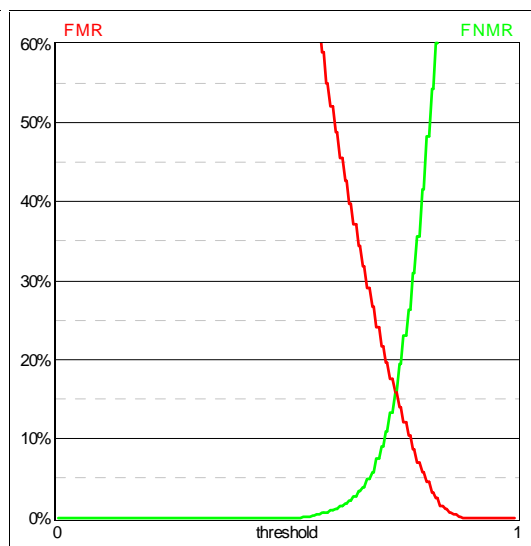

FMR(t) and $\operatorname{FNMR}(\mathrm{t})$

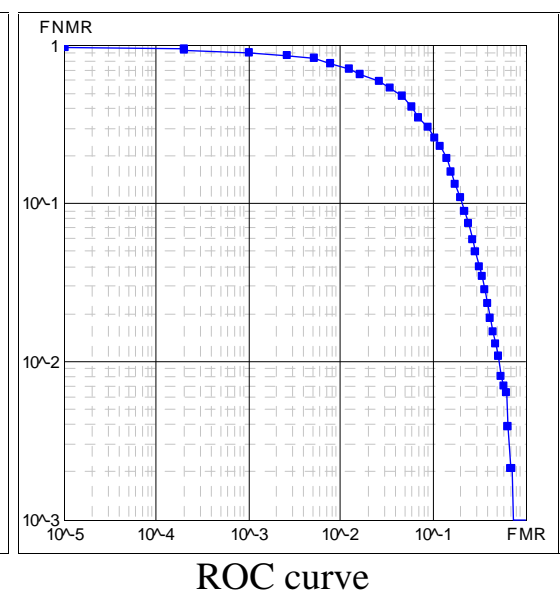




\section{Algorithm $K r d l$ on database $D B 1 A$}

Average enroll time: 1.00 seconds

Average match time: 1.06 seconds

\begin{tabular}{|c|c|c|c|c|}
\hline REJ $_{\text {ENROLL }}$ & NGRA & NIRA & REJ $_{\text {NGRA }}$ & REJ $_{\text {NIRA }}$ \\
\hline $6.43 \%(\mathrm{~F}: 43 \mathrm{~T}: 2 \mathrm{C}: 0)$ & 2644 & 4841 & $4.43 \%(\mathrm{~F}: 113 \mathrm{~T}: 4 \mathrm{C}: 0)$ & $7.77 \%(\mathrm{~F}: 376 \mathrm{~T}: 0 \mathrm{C}: 0)$ \\
\hline
\end{tabular}

\begin{tabular}{|c|c|c|c|}
\hline EER & EER $^{*}$ & ZeroFMR & ZeroFNMR \\
\hline $10.66 \%$ & $7.35 \%(7.35 \%-7.36 \%)$ & $24.51 \%$ & $100.00 \%$ \\
\hline
\end{tabular}

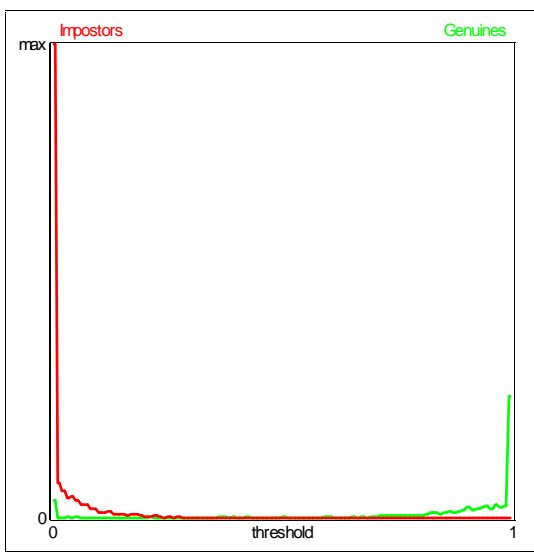

Score distributions

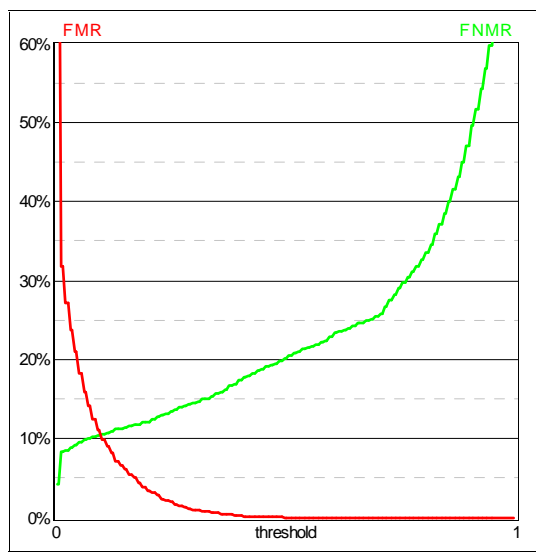

FMR(t) and FNMR(t)

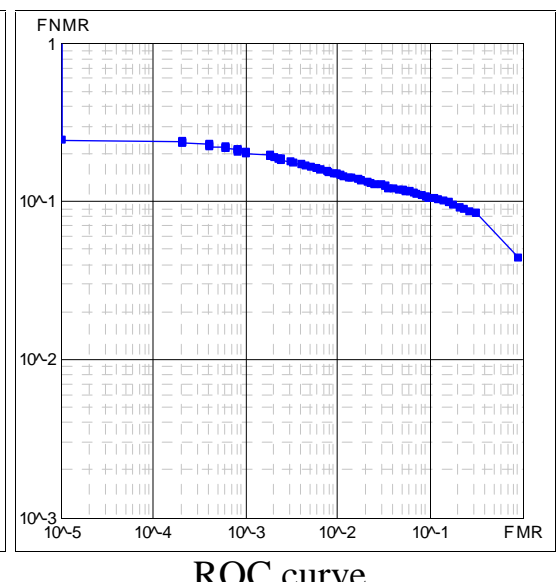

ROC curve

\section{Algorithm $K r d l$ on database $D B 2$ A}

Average enroll time: 1.16 seconds

Average match time: 2.88 seconds

\begin{tabular}{|c|c|c|c|c|}
\hline REJ $_{\text {ENROLL }}$ & NGRA & NIRA & REJ $_{\text {NGRA }}$ & REJ $_{\text {NIRA }}$ \\
\hline $3.29 \%(\mathrm{~F}: 22 \mathrm{~T}: 1 \mathrm{C}: 0)$ & 2702 & 4942 & $1.85 \%(\mathrm{~F}: 46 \mathrm{~T}: 4 \mathrm{C}: 0)$ & $5.81 \%(\mathrm{~F}: 286 \mathrm{~T}: 1 \mathrm{C}: 0)$ \\
\hline
\end{tabular}

\begin{tabular}{|c|c|c|c|}
\hline EER & EER* & ZeroFMR & ZeroFNMR \\
\hline $8.83 \%(8.82 \%-8.85 \%)$ & $7.53 \%(7.52 \%-7.54 \%)$ & $22.13 \%$ & $100.00 \%$ \\
\hline
\end{tabular}

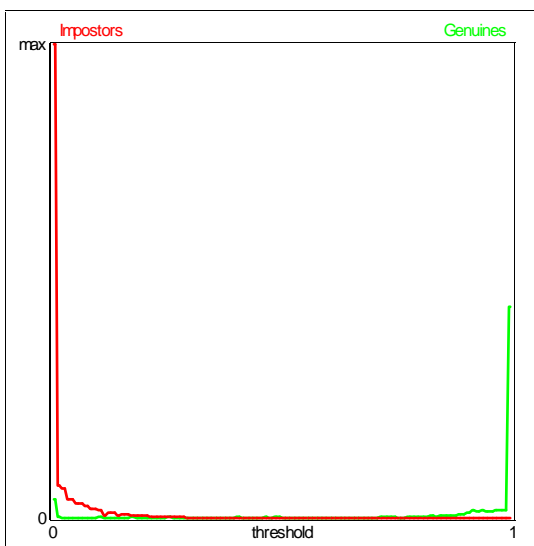

Score distributions

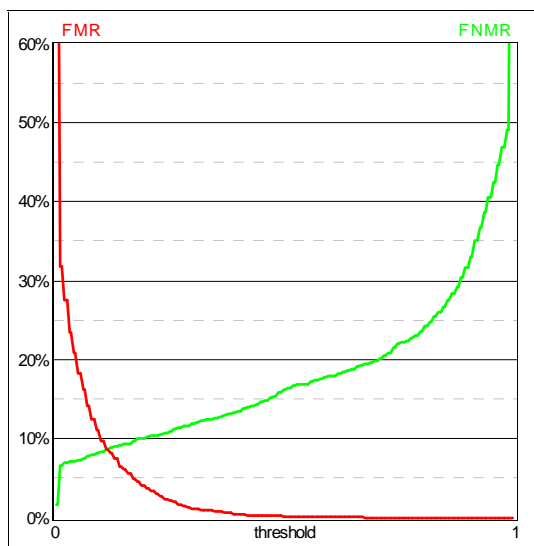

FMR $(\mathrm{t})$ and $\operatorname{FNMR}(\mathrm{t})$

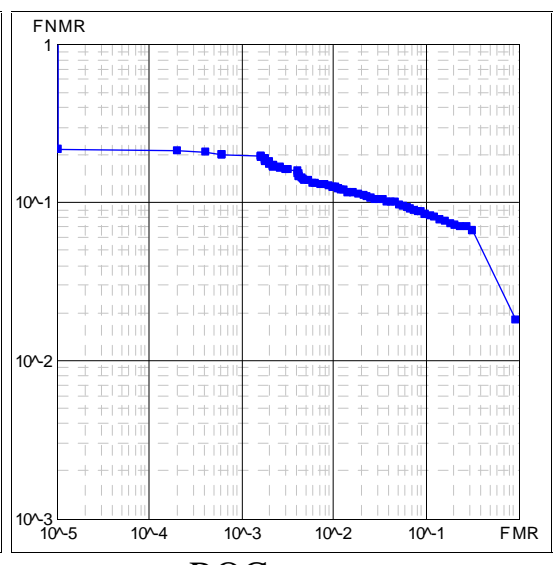

ROC curve 


\section{Algorithm $K r d l$ on database $D B 3 \_A$}

Average enroll time: 1.48 seconds Average match time: 1.60 seconds

\begin{tabular}{|c|c|c|c|c|}
\hline $\mathbf{R E J}_{\text {ENROLL }}$ & NGRA & NIRA & REJ $_{\text {NGRA }}$ & $\mathbf{R E J}_{\text {NIRA }}$ \\
\hline $6.86 \%(\mathrm{~F}: 48 \mathrm{~T}: 0 \mathrm{C}: 0)$ & 2637 & 4691 & $5.65 \%$ (F:149 T:0 C:0) & $4.82 \%$ (F:226 T:0 C:0) \\
\hline EER & \multicolumn{2}{|c|}{ EER* $^{*}$} & ZeroFMR & ZeroFNMR \\
\hline $12.20 \%(12.19 \%-12.21 \%)$ & \multicolumn{2}{|c|}{$8.03 \%(7.97 \%-8.08 \%)$} & $22.83 \%$ & $100.00 \%$ \\
\hline
\end{tabular}

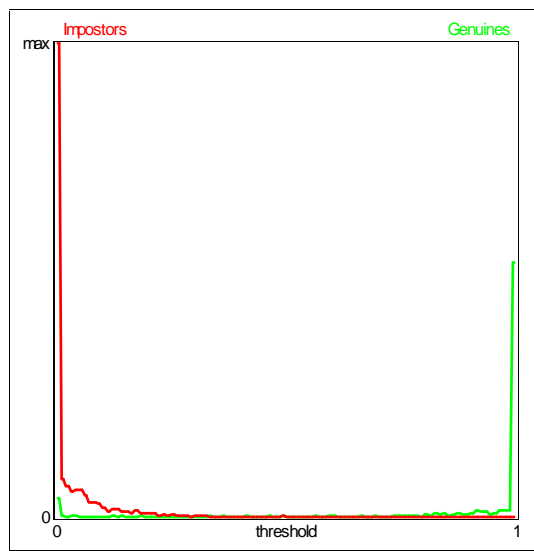

Score distributions

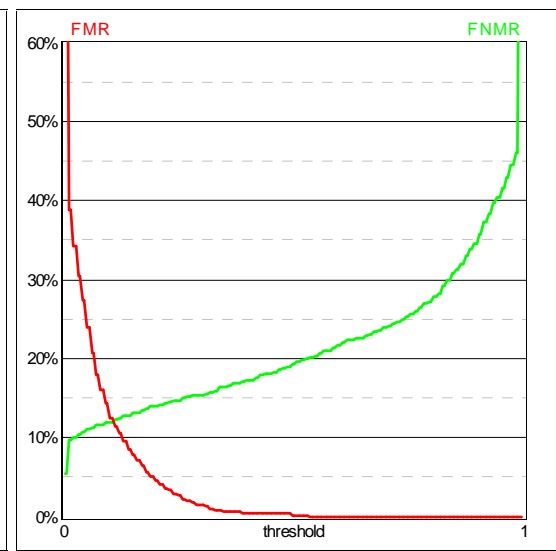

FMR(t) and FNMR(t)

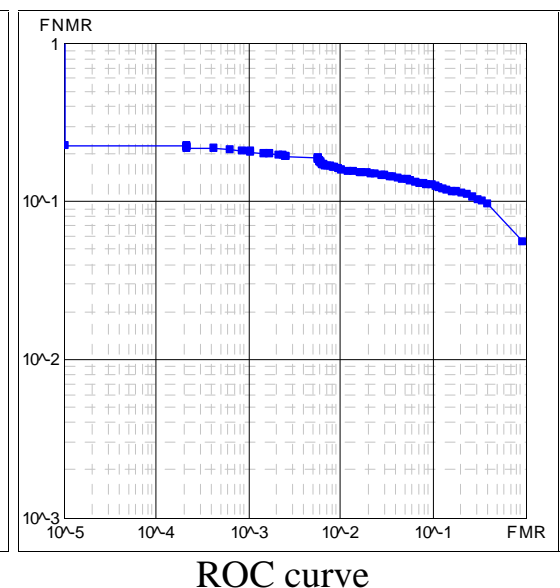

ROC curve

\section{Algorithm $K r d l$ on database $D B 4 \_A$}

Average enroll time: 0.70 seconds

Average match time: 0.79 seconds

\begin{tabular}{|c|c|c|c|c|}
\hline REJ $_{\text {ENROLL }}$ & NGRA & NIRA & REJ $_{\text {NGRA }}$ & $\mathbf{R E J}_{\text {NIRA }}$ \\
\hline $10.86 \%(\mathrm{~F}: 76 \mathrm{~T}: 0 \mathrm{C}: 0)$ & 2488 & 4477 & $6.11 \%(\mathrm{~F}: 152 \mathrm{~T}: 0 \mathrm{C}: 0)$ & $12.53 \%$ (F:561 T:0 C:0) \\
\hline EER & \multicolumn{2}{|c|}{ EER* } & ZeroFMR & ZeroFNMR \\
\hline $12.08 \%(12.06 \%-12.10 \%)$ & \multicolumn{2}{|c|}{$7.46 \%(7.43 \%-7.49 \%)$} & $40.19 \%$ & $100.00 \%$ \\
\hline
\end{tabular}

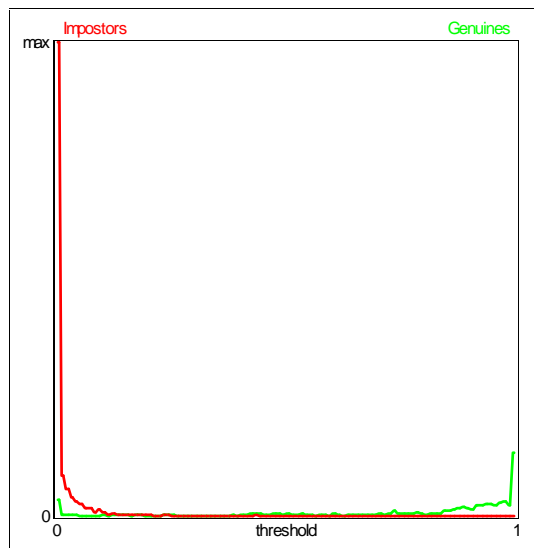

Score distributions

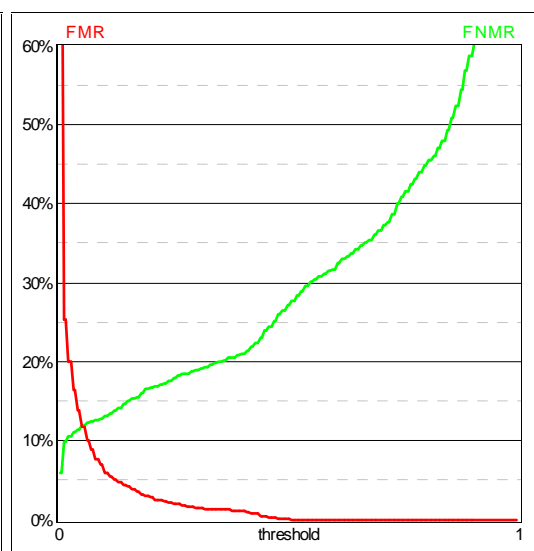

FMR(t) and $\operatorname{FNMR}(\mathrm{t})$

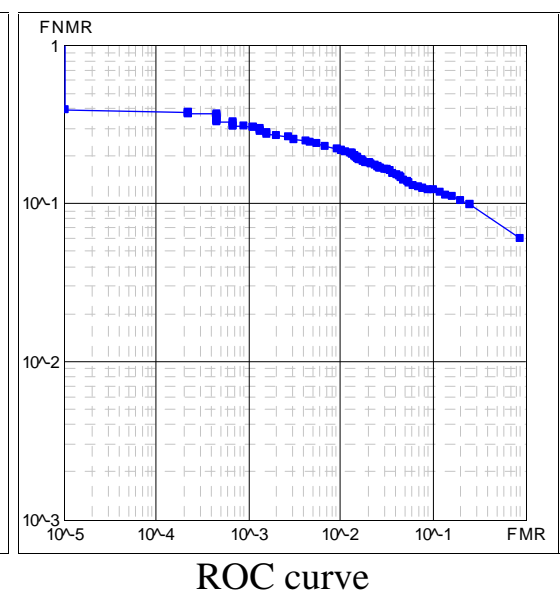




\section{Algorithm Ncmi on database $D B 1 A$}

Average enroll time: 1.13 seconds

Average match time: 1.34 seconds

\begin{tabular}{|c|c|c|c|c|}
\hline REJ $_{\text {ENROLL }}$ & NGRA & NIRA & REJ $_{\text {NGRA }}$ & REJ $_{\text {NIRA }}$ \\
\hline $0.00 \%(\mathrm{~F}: 0 \mathrm{~T}: 0 \mathrm{C}: 0)$ & 2800 & 4950 & $0.00 \%(\mathrm{~F}: 0 \mathrm{~T}: 0 \mathrm{C}: 0)$ & $0.18 \%(\mathrm{~F}: 9 \mathrm{~T}: 0 \mathrm{C}: 0)$ \\
\hline
\end{tabular}

\begin{tabular}{|c|c|c|c|}
\hline EER & EER* & ZeroFMR & ZeroFNMR \\
\hline $49.11 \%(48.82 \%-49.39 \%)$ & $49.15 \%(48.82 \%-49.48 \%)$ & $100.00 \%$ & $99.82 \%$ \\
\hline
\end{tabular}

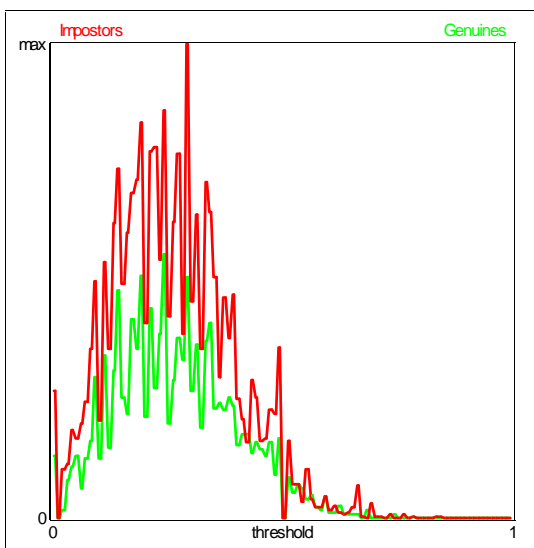

Score distributions

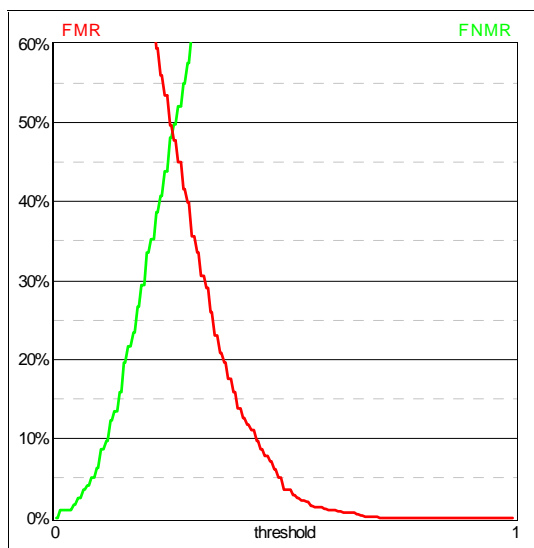

FMR (t) and FNMR(t)

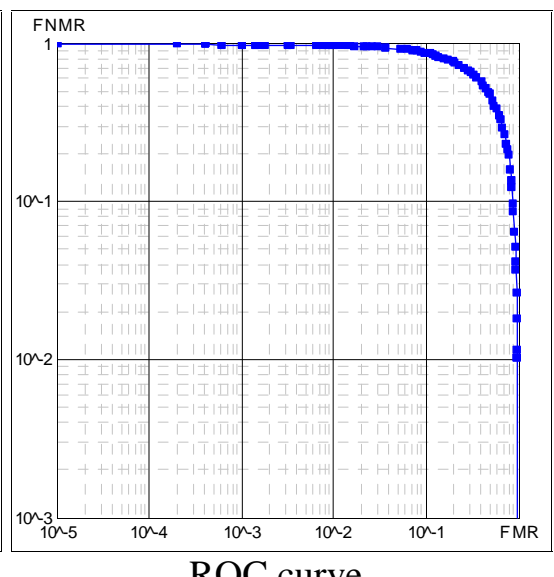

ROC curve

\section{Algorithm Ncmi on database $D B 2 \_A$}

Average enroll time: 1.28 seconds

Average match time: 1.57 seconds

\begin{tabular}{|c|c|c|c|c|}
\hline REJ $_{\text {ENROLL }}$ & NGRA & NIRA & REJ $_{\text {NGRA }}$ & REJ $_{\text {NIRA }}$ \\
\hline $0.00 \%(\mathrm{~F}: 0 \mathrm{~T}: 0 \mathrm{C}: 0)$ & 2800 & 4950 & $0.00 \%(\mathrm{~F}: 0 \mathrm{~T}: 0 \mathrm{C}: 0)$ & $0.00 \%(\mathrm{~F}: 0 \mathrm{~T}: 0 \mathrm{C}: 0)$ \\
\hline
\end{tabular}

\begin{tabular}{|c|c|c|c|}
\hline EER & EER* & ZeroFMR & ZeroFNMR \\
\hline $46.15 \%(45.82 \%-46.48 \%)$ & $46.15 \%(45.82 \%-46.48 \%)$ & $100.00 \%$ & $100.00 \%$ \\
\hline
\end{tabular}

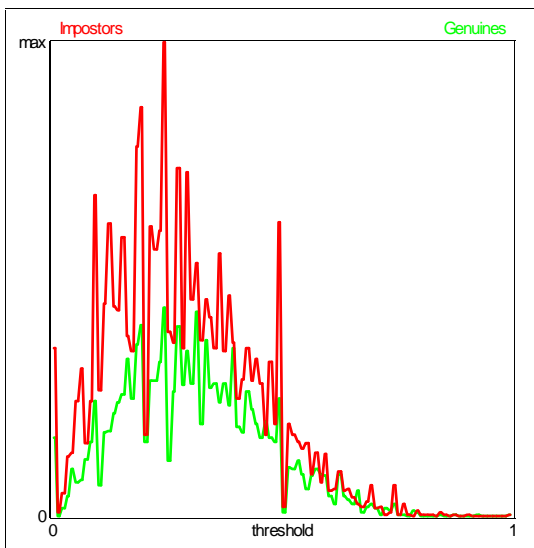

Score distributions

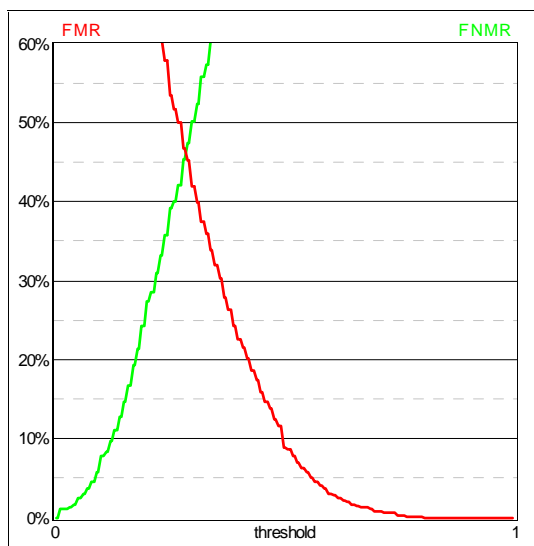

FMR $(t)$ and $F N M R(t)$

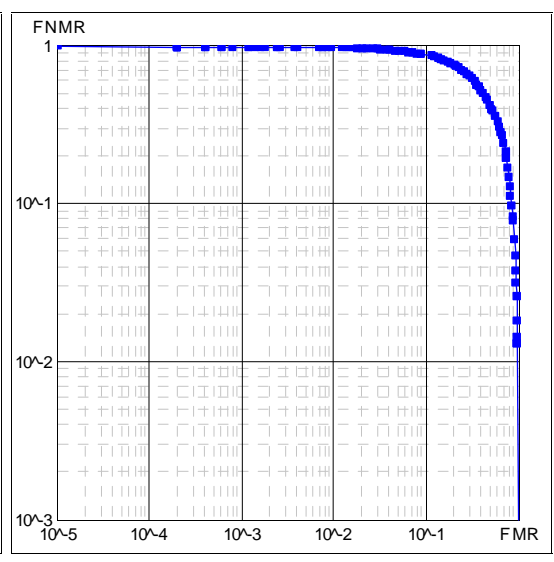

ROC curve 


\section{Algorithm Ncmi on database $D B 3 \_A$}

Average enroll time: 2.25 seconds

Average match time: 2.75 seconds

\begin{tabular}{|c|c|c|c|c|}
\hline REJ $_{\text {ENROLL }}$ & NGRA & NIRA & REJ $_{\text {NGRA }}$ & REJ $_{\text {NIRA }}$ \\
\hline $0.00 \%(\mathrm{~F}: 0 \mathrm{~T}: 0 \mathrm{C}: 0)$ & 2800 & 4950 & $0.00 \%(\mathrm{~F}: 0 \mathrm{~T}: 0 \mathrm{C}: 0)$ & $0.02 \%(\mathrm{~F}: 1 \mathrm{~T}: 0 \mathrm{C}: 0)$ \\
\hline
\end{tabular}

\begin{tabular}{|c|c|c|c|}
\hline EER & EER* & ZeroFMR & ZeroFNMR \\
\hline $47.43 \%(45.76 \%-49.11 \%)$ & $47.44 \%(45.77 \%-49.11 \%)$ & $100.00 \%$ & $99.98 \%$ \\
\hline
\end{tabular}

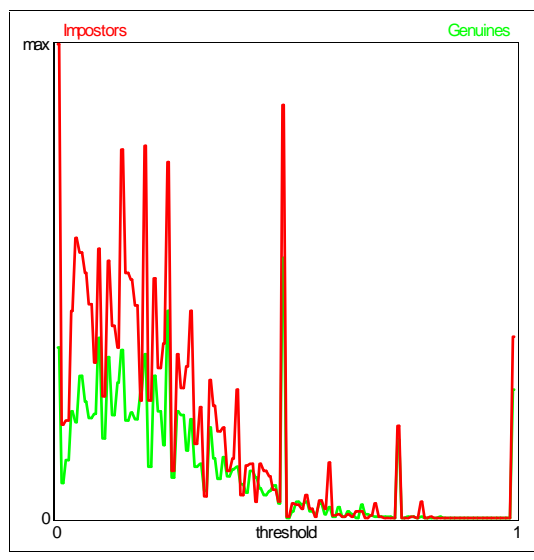

Score distributions

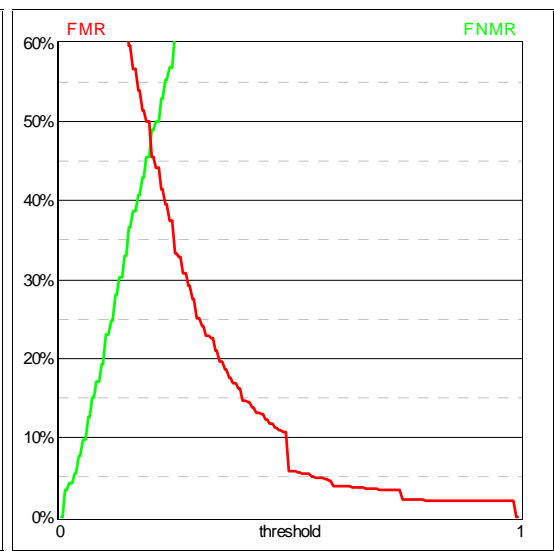

FMR(t) and FNMR(t)

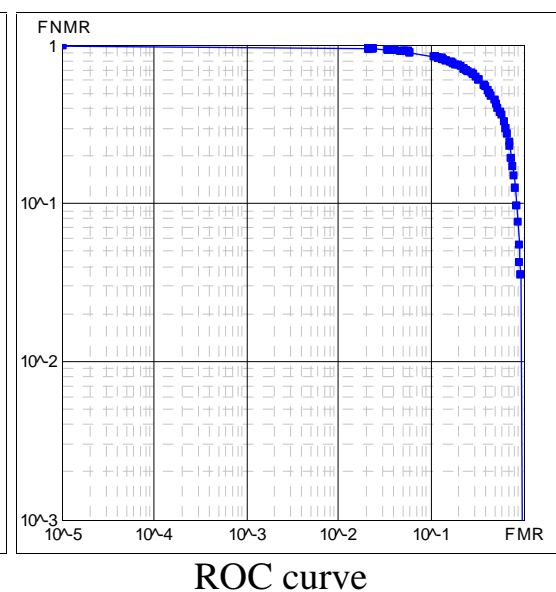

ROC curve

\section{Algorithm Ncmi on database DB4_A}

Average enroll time: 1.08 seconds

Average match time: 1.19 seconds

\begin{tabular}{|c|c|c|c|c|}
\hline REJ $_{\text {ENROLL }}$ & NGRA & NIRA & $\mathbf{R E J}_{\text {NGRA }}$ & $\mathbf{R E J}_{\text {NIRA }}$ \\
\hline $0.00 \%(\mathrm{~F}: 0 \mathrm{~T}: 0 \mathrm{C}: 0)$ & 2800 & 4950 & $0.18 \%(\mathrm{~F}: 5 \mathrm{~T}: 0 \mathrm{C}: 0)$ & $0.28 \%(\mathrm{~F}: 14 \mathrm{~T}: 0 \mathrm{C}: 0)$ \\
\hline EER & \multicolumn{2}{|c|}{ EER* } & ZeroFMR & ZeroFNMR \\
\hline $48.67 \%(48.67 \%-48.68 \%)$ & \multicolumn{2}{|c|}{$48.77 \%(48.73 \%-48.80 \%)$} & $99.96 \%$ & $100.00 \%$ \\
\hline
\end{tabular}

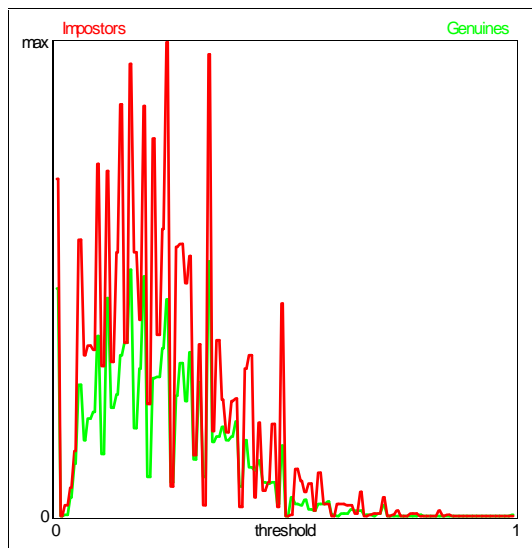

Score distributions

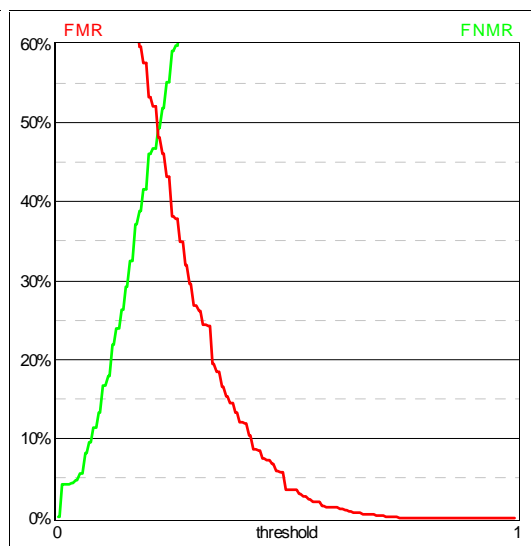

FMR(t) and $\operatorname{FNMR}(\mathrm{t})$

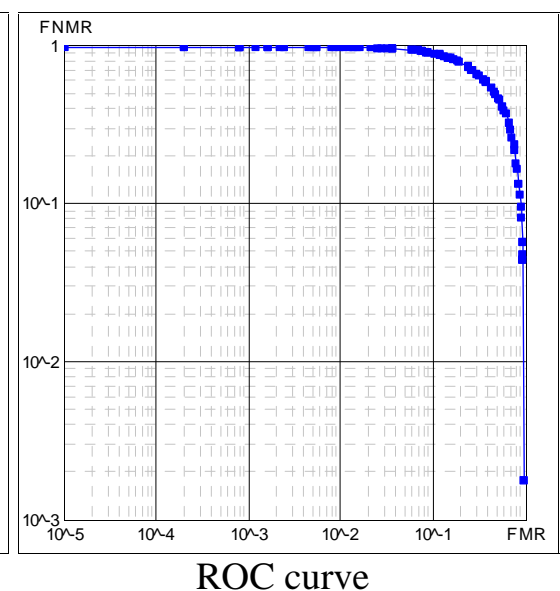




\section{Algorithm Sag1 on database $D B 1 A$}

Average enroll time: 2.48 seconds

Average match time: 0.96 seconds

\begin{tabular}{|c|c|c|c|c|}
\hline REJ $_{\text {ENROLL }}$ & NGRA & NIRA & REJ $_{\text {NGRA }}$ & REJ $_{\text {NIRA }}$ \\
\hline $0.00 \%(\mathrm{~F}: 0 \mathrm{~T}: 0 \mathrm{C}: 0)$ & 2800 & 4950 & $0.00 \%(\mathrm{~F}: 0 \mathrm{~T}: 0 \mathrm{C}: 0)$ & $0.00 \%(\mathrm{~F}: 0 \mathrm{~T}: 0 \mathrm{C}: 0)$ \\
\hline
\end{tabular}

\begin{tabular}{|c|c|c|c|}
\hline EER & EER* & ZeroFMR & ZeroFNMR \\
\hline $0.67 \%(0.67 \%-0.68 \%)$ & $0.67 \%(0.67 \%-0.68 \%)$ & $2.11 \%$ & $53.13 \%$ \\
\hline
\end{tabular}

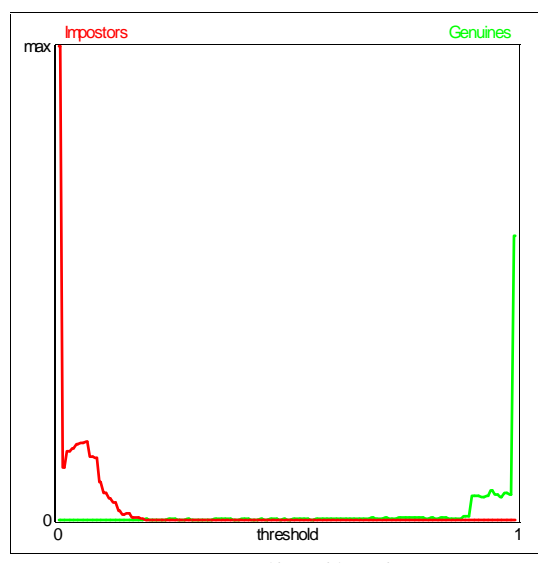

Score distributions

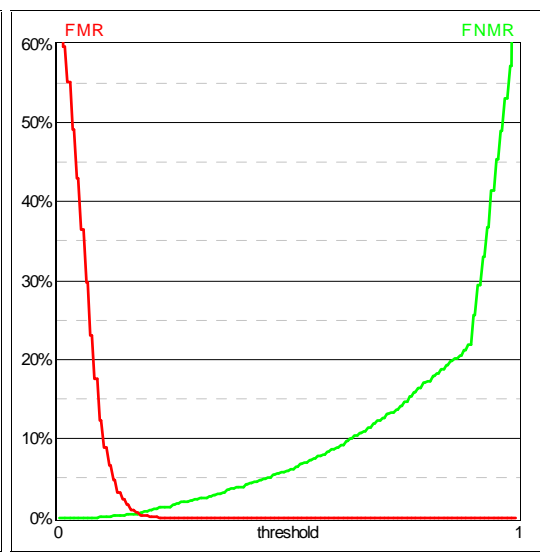

FMR (t) and FNMR(t)

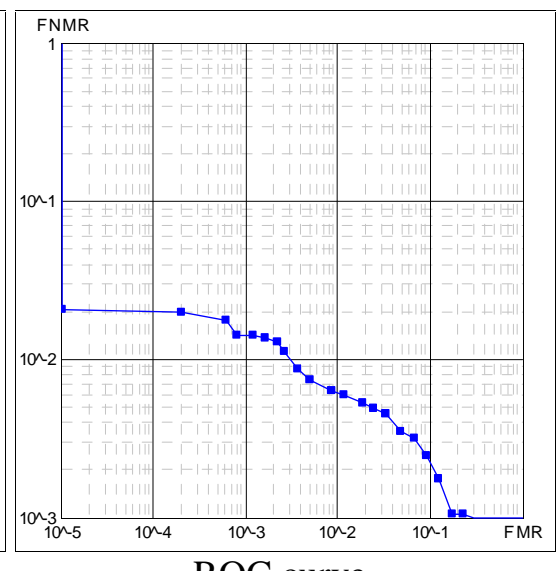

ROC curve

\section{Algorithm Sag1 on database $D B 2 \_A$}

Average enroll time: 2.63 seconds

Average match time: 1.03 seconds

\begin{tabular}{|c|c|c|c|c|}
\hline REJ $_{\text {ENROLL }}$ & NGRA & NIRA & REJ $_{\text {NGRA }}$ & REJ $_{\text {NIRA }}$ \\
\hline $0.00 \%(\mathrm{~F}: 0 \mathrm{~T}: 0 \mathrm{C}: 0)$ & 2800 & 4950 & $0.00 \%(\mathrm{~F}: 0 \mathrm{~T}: 0 \mathrm{C}: 0)$ & $0.00 \%(\mathrm{~F}: 0 \mathrm{~T}: 0 \mathrm{C}: 0)$ \\
\hline
\end{tabular}

\begin{tabular}{|c|c|c|c|}
\hline EER & EER* & ZeroFMR & ZeroFNMR \\
\hline $0.61 \%$ & $0.61 \%$ & $1.36 \%$ & $50.69 \%$ \\
\hline
\end{tabular}

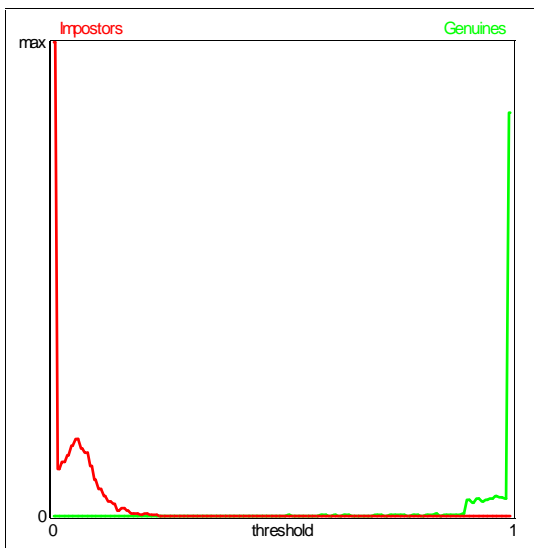

Score distributions

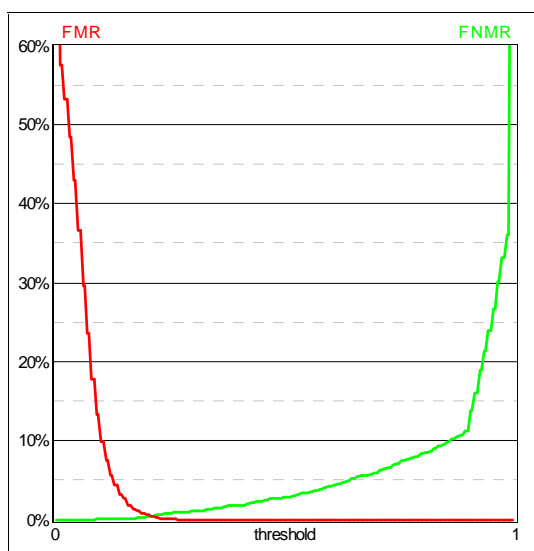

FMR $(t)$ and $F N M R(t)$

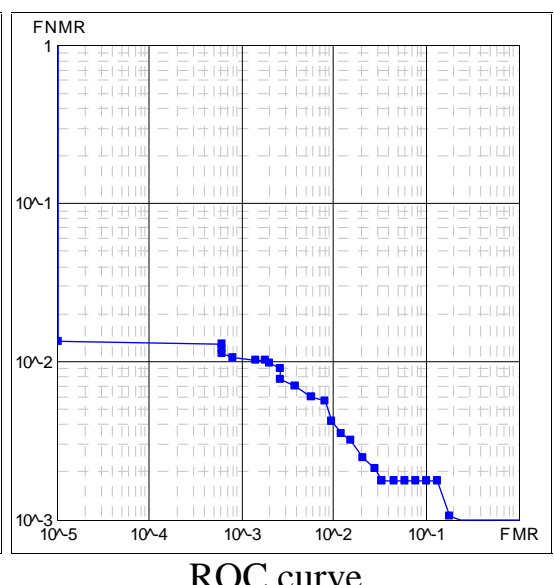


Algorithm Sag1 on database DB3_A

Average enroll time: 5.70 seconds

Average match time: 2.13 seconds

\begin{tabular}{|c|c|c|c|c|}
\hline REJ $_{\text {ENROLL }}$ & NGRA & NIRA & REJ $_{\text {NGRA }}$ & REJ $_{\text {NIRA }}$ \\
\hline $0.00 \%(\mathrm{~F}: 0 \mathrm{~T}: 0 \mathrm{C}: 0)$ & 2800 & 4950 & $0.00 \%(\mathrm{~F}: 0 \mathrm{~T}: 0 \mathrm{C}: 0)$ & $0.00 \%(\mathrm{~F}: 0 \mathrm{~T}: 0 \mathrm{C}: 0)$ \\
\hline
\end{tabular}

\begin{tabular}{|c|c|c|c|}
\hline EER & EER* & ZeroFMR & ZeroFNMR \\
\hline $3.64 \%$ & $3.64 \%$ & $6.82 \%$ & $100.00 \%$ \\
\hline
\end{tabular}

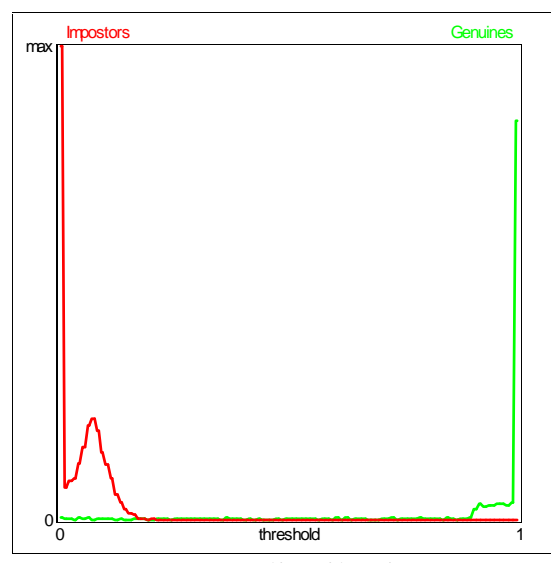

Score distributions

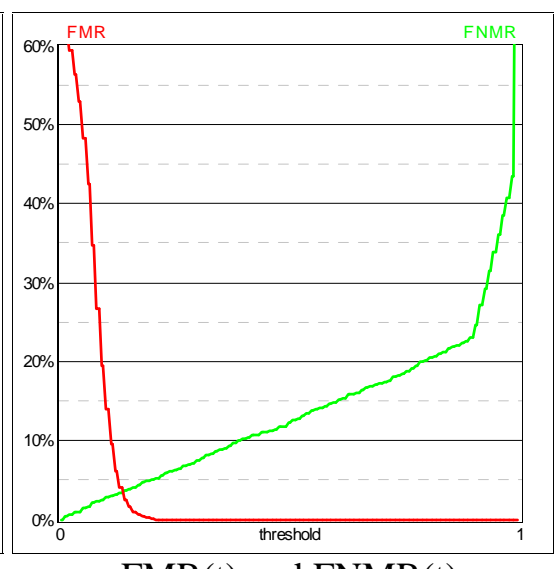

FMR(t) and FNMR(t)

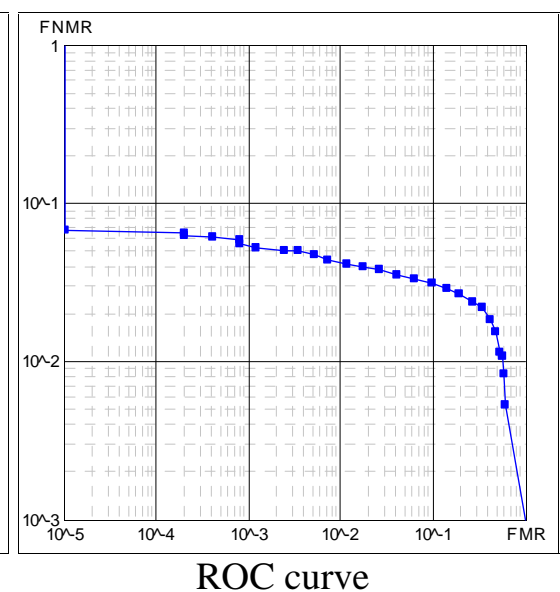

ROC curve

\section{Algorithm Sag1 on database $D B 4 \_A$}

Average enroll time: 1.90 seconds

Average match time: 0.77 seconds

\begin{tabular}{|c|c|c|c|c|}
\hline REJ $_{\text {ENROLL }}$ & NGRA & NIRA & $\mathbf{R E J}_{\text {NGRA }}$ & $\mathbf{R E J}_{\text {NIRA }}$ \\
\hline $0.00 \%(\mathrm{~F}: 0 \mathrm{~T}: 0 \mathrm{C}: 0)$ & 2800 & 4950 & $0.00 \%$ (F:0 T:0 C:0) & $0.00 \%$ (F:0 T:0 C:0) \\
\hline EER & \multicolumn{2}{|c|}{ EER* } & ZeroFMR & ZeroFNMR \\
\hline $1.99 \%(1.98 \%-2.00 \%)$ & \multicolumn{2}{|c|}{$1.99 \%(1.98 \%-2.00 \%)$} & $6.71 \%$ & $100.00 \%$ \\
\hline
\end{tabular}

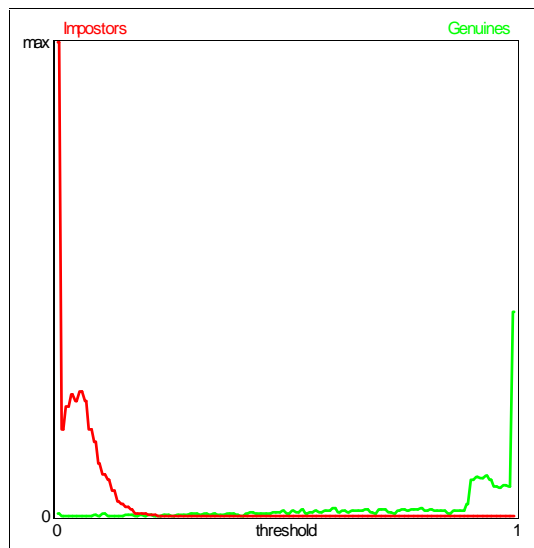

Score distributions

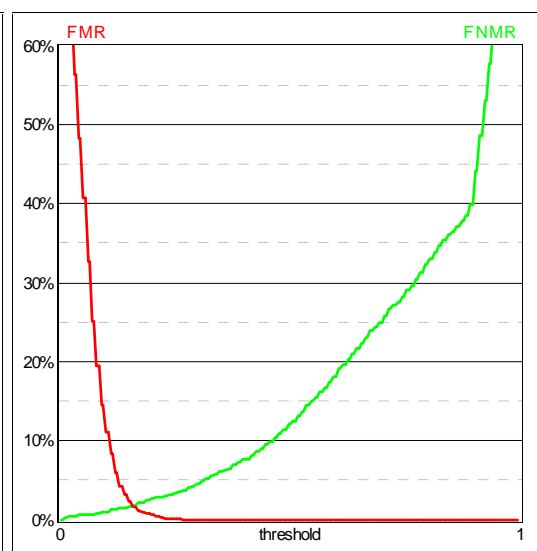

FMR(t) and FNMR(t)

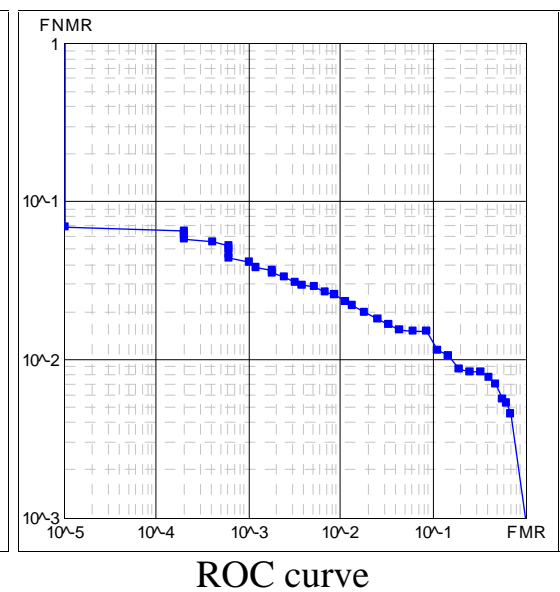




\section{Algorithm Sag2 on database $D B 1 A$}

Average enroll time: 0.88 seconds

Average match time: 0.88 seconds

\begin{tabular}{|c|c|c|c|c|}
\hline REJ $_{\text {ENROLL }}$ & NGRA & NIRA & REJ $_{\text {NGRA }}$ & REJ $_{\text {NIRA }}$ \\
\hline $0.00 \%(\mathrm{~F}: 0 \mathrm{~T}: 0 \mathrm{C}: 0)$ & 2800 & 4950 & $0.00 \%(\mathrm{~F}: 0 \mathrm{~T}: 0 \mathrm{C}: 0)$ & $0.00 \%(\mathrm{~F}: 0 \mathrm{~T}: 0 \mathrm{C}: 0)$ \\
\hline
\end{tabular}

\begin{tabular}{|c|c|c|c|}
\hline EER & EER* & ZeroFMR & ZeroFNMR \\
\hline $1.17 \%(1.15 \%-1.18 \%)$ & $1.17 \%(1.15 \%-1.18 \%)$ & $3.07 \%$ & $74.57 \%$ \\
\hline
\end{tabular}

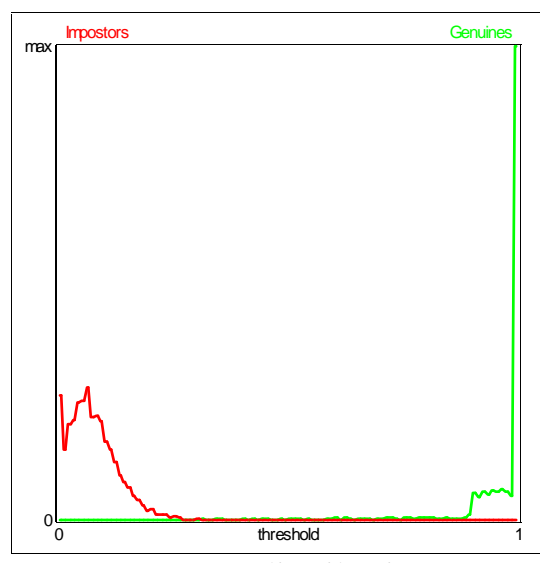

Score distributions

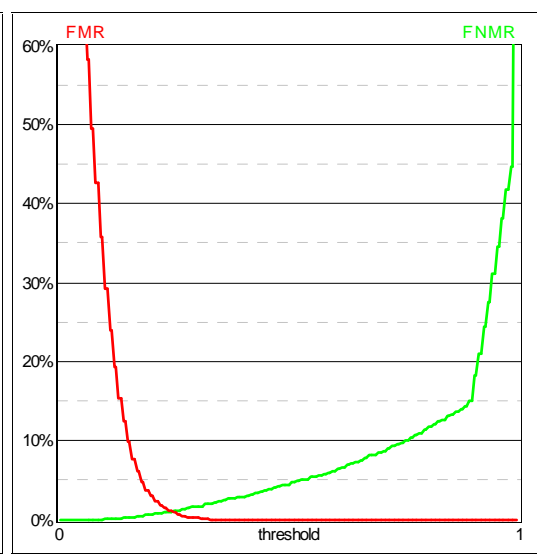

FMR (t) and FNMR(t)

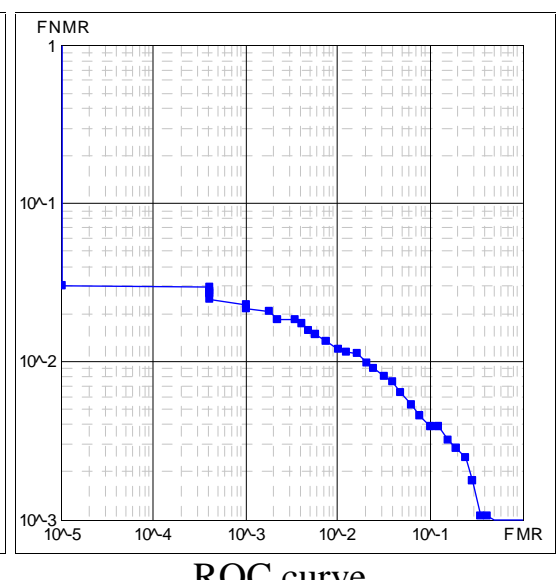

ROC curve

\section{Algorithm Sag2 on database $D B 2 \_A$}

Average enroll time: 0.93 seconds

Average match time: 0.93 seconds

\begin{tabular}{|c|c|c|c|c|}
\hline REJ $_{\text {ENROLL }}$ & NGRA & NIRA & REJ $_{\text {NGRA }}$ & REJ $_{\text {NIRA }}$ \\
\hline $0.00 \%(\mathrm{~F}: 0 \mathrm{~T}: 0 \mathrm{C}: 0)$ & 2800 & 4950 & $0.00 \%(\mathrm{~F}: 0 \mathrm{~T}: 0 \mathrm{C}: 0)$ & $0.00 \%(\mathrm{~F}: 0 \mathrm{~T}: 0 \mathrm{C}: 0)$ \\
\hline
\end{tabular}

\begin{tabular}{|c|c|c|c|}
\hline EER & EER* & ZeroFMR & ZeroFNMR \\
\hline $0.82 \%$ & $0.82 \%$ & $2.14 \%$ & $100.00 \%$ \\
\hline
\end{tabular}

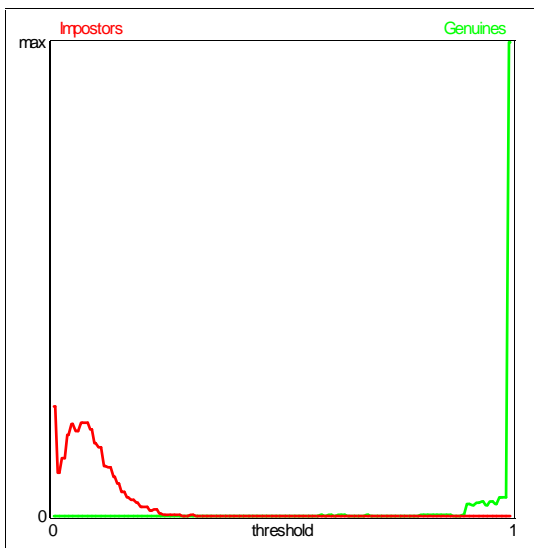

Score distributions

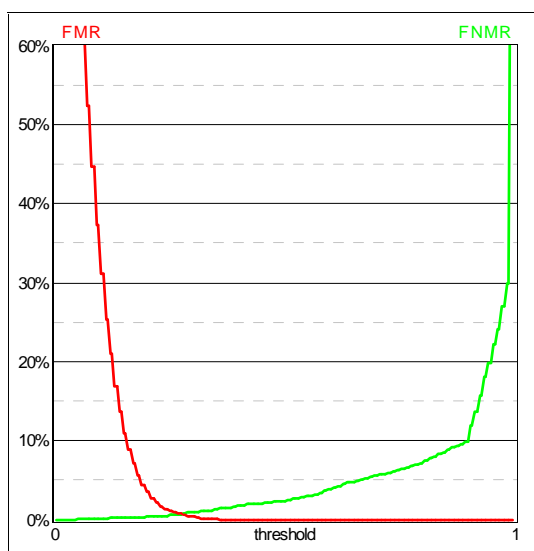

FMR $(t)$ and $F N M R(t)$

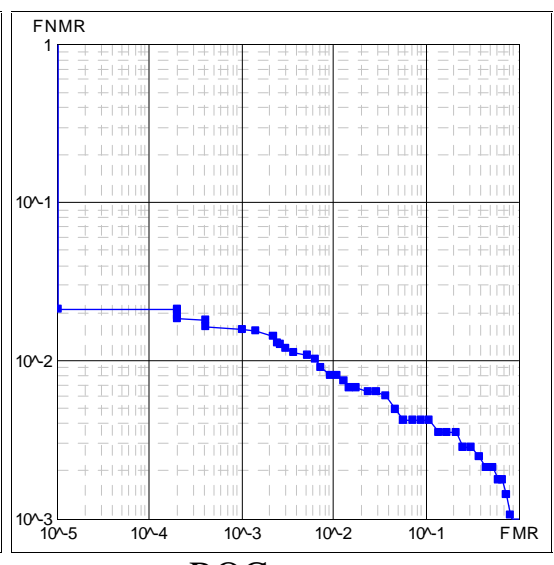

ROC curve 
Algorithm Sag2 on database DB3_A

Average enroll time: 1.94 seconds

Average match time: 1.94 seconds

\begin{tabular}{|c|c|c|c|c|}
\hline REJ $_{\text {ENROLL }}$ & NGRA & NIRA & REJ $_{\text {NGRA }}$ & REJ $_{\text {NIRA }}$ \\
\hline $0.00 \%(\mathrm{~F}: 0 \mathrm{~T}: 0 \mathrm{C}: 0)$ & 2800 & 4950 & $0.00 \%(\mathrm{~F}: 0 \mathrm{~T}: 0 \mathrm{C}: 0)$ & $0.00 \%(\mathrm{~F}: 0 \mathrm{~T}: 0 \mathrm{C}: 0)$ \\
\hline
\end{tabular}

\begin{tabular}{|c|c|c|c|}
\hline EER & EER* & ZeroFMR & ZeroFNMR \\
\hline $4.01 \%(3.98 \%-4.04 \%)$ & $4.01 \%(3.98 \%-4.04 \%)$ & $9.50 \%$ & $100.00 \%$ \\
\hline
\end{tabular}

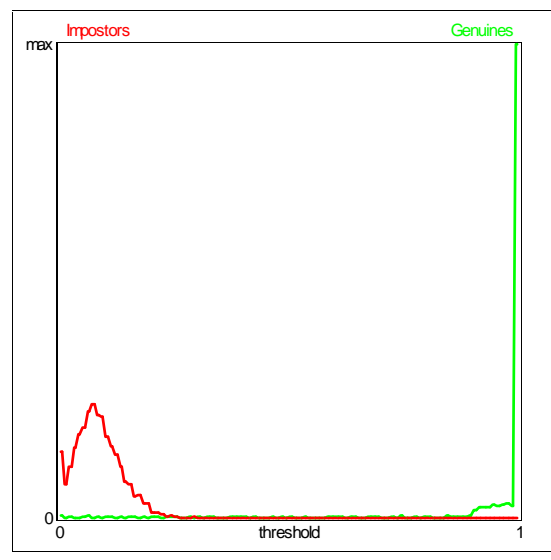

Score distributions

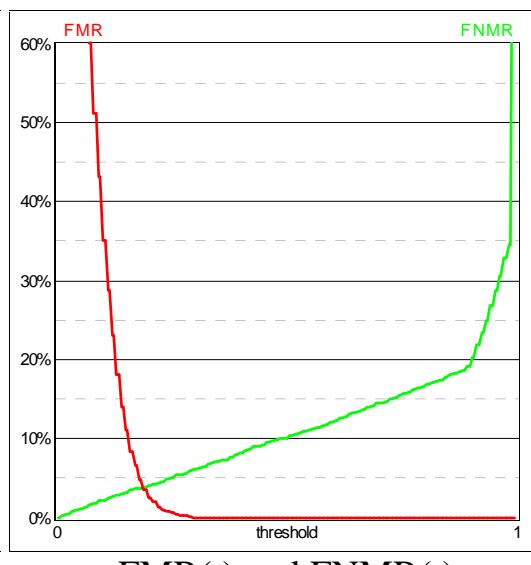

FMR(t) and FNMR(t)

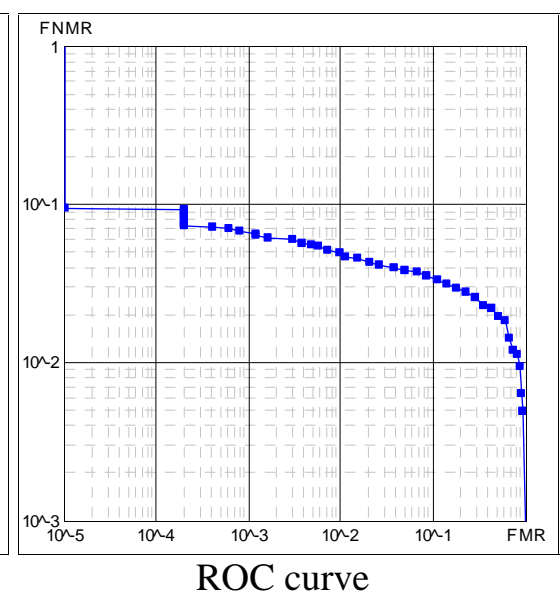

\section{Algorithm Sag2 on database $D B 4 \_A$}

Average enroll time: 0.69 seconds

Average match time: 0.69 seconds

\begin{tabular}{|c|c|c|c|c|}
\hline REJ $_{\text {ENROLL }}$ & NGRA & NIRA & REJ $_{\text {NGRA }}$ & REJ $_{\text {NIRA }}$ \\
\hline $0.00 \%(\mathrm{~F}: 0 \mathrm{~T}: 0 \mathrm{C}: 0)$ & 2800 & 4950 & $0.00 \%(\mathrm{~F}: 0 \mathrm{~T}: 0 \mathrm{C}: 0)$ & $0.00 \%(\mathrm{~F}: 0 \mathrm{~T}: 0 \mathrm{C}: 0)$ \\
\hline
\end{tabular}

\begin{tabular}{|c|c|c|c|}
\hline EER & EER* & ZeroFMR & ZeroFNMR \\
\hline $3.11 \%$ & $3.11 \%$ & $10.57 \%$ & $100.00 \%$ \\
\hline
\end{tabular}

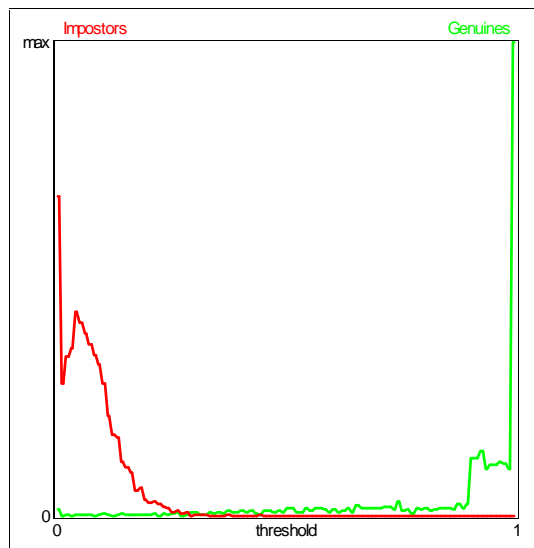

Score distributions

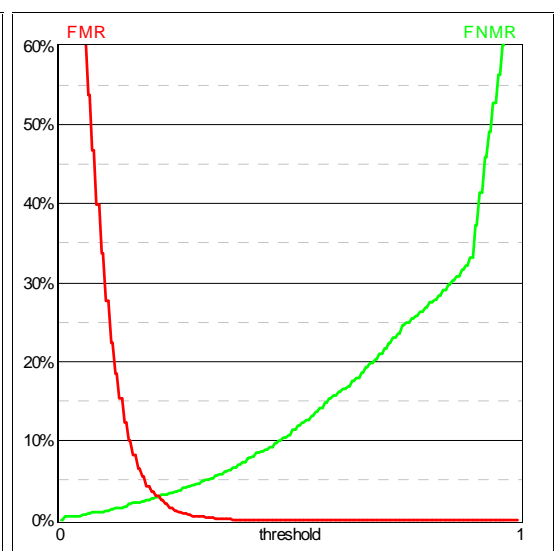

FMR(t) and FNMR(t)

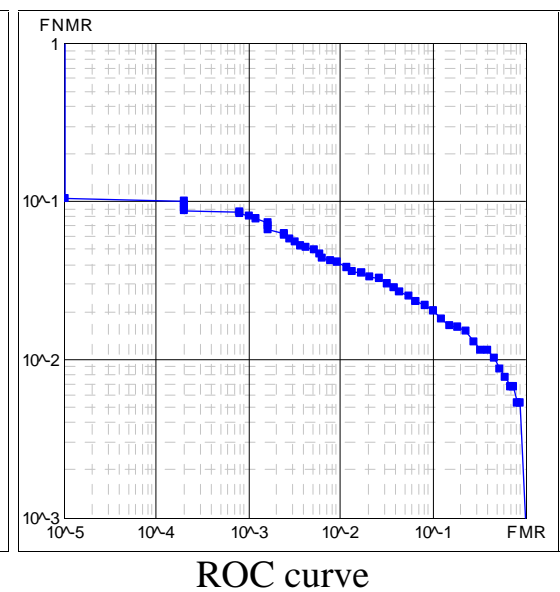




\section{Algorithm Uinh on database $D B 1 A$}

Average enroll time: 0.53 seconds

Average match time: 0.56 seconds

\begin{tabular}{|c|c|c|c|c|}
\hline REJ $_{\text {ENROLL }}$ & NGRA & NIRA & REJ $_{\text {NGRA }}$ & REJ $_{\text {NIRA }}$ \\
\hline $1.71 \%(\mathrm{~F}: 12 \mathrm{~T}: 0 \mathrm{C}: 0)$ & 2745 & 4911 & $1.71 \%(\mathrm{~F}: 47 \mathrm{~T}: 0 \mathrm{C}: 0)$ & $6.96 \%(\mathrm{~F}: 342 \mathrm{~T}: 0 \mathrm{C}: 0)$ \\
\hline
\end{tabular}

\begin{tabular}{|c|c|c|c|}
\hline EER & EER* & ZeroFMR & ZeroFNMR \\
\hline $21.02 \%(20.91 \%-21.14 \%)$ & $20.65 \%(20.64 \%-20.66 \%)$ & $82.00 \%$ & $100.00 \%$ \\
\hline
\end{tabular}

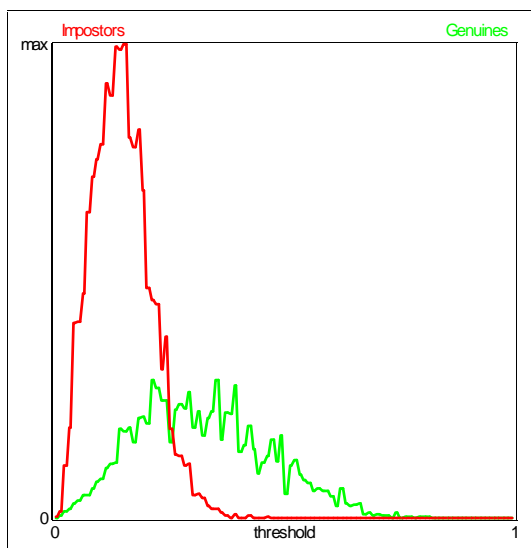

Score distributions

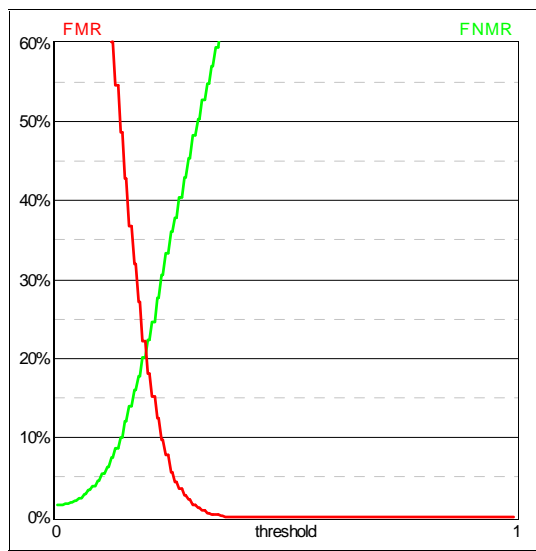

FMR (t) and FNMR(t)

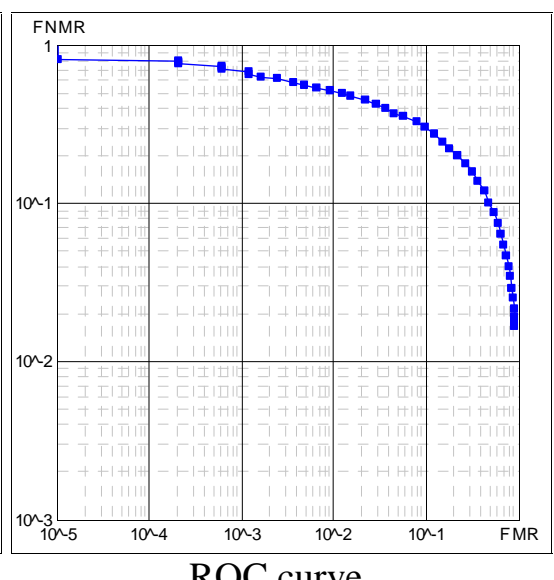

ROC curve

\section{Algorithm Uinh on database $D B 2$ A}

Average enroll time: 0.60 seconds

Average match time: 0.65 seconds

\begin{tabular}{|c|c|c|c|c|}
\hline REJ $_{\text {ENROLL }}$ & NGRA & NIRA & REJ $_{\text {NGRA }}$ & REJ $_{\text {NIRA }}$ \\
\hline $0.86 \%(\mathrm{~F}: 6 \mathrm{~T}: 0 \mathrm{C}: 0)$ & 2768 & 4922 & $1.45 \%(\mathrm{~F}: 40 \mathrm{~T}: 0 \mathrm{C}: 0)$ & $5.57 \%(\mathrm{~F}: 274 \mathrm{~T}: 0 \mathrm{C}: 0)$ \\
\hline
\end{tabular}

\begin{tabular}{|c|c|c|c|}
\hline EER & EER* & ZeroFMR & ZeroFNMR \\
\hline $15.22 \%(15.21 \%-15.24 \%)$ & $14.70 \%$ & $56.11 \%$ & $100.00 \%$ \\
\hline
\end{tabular}

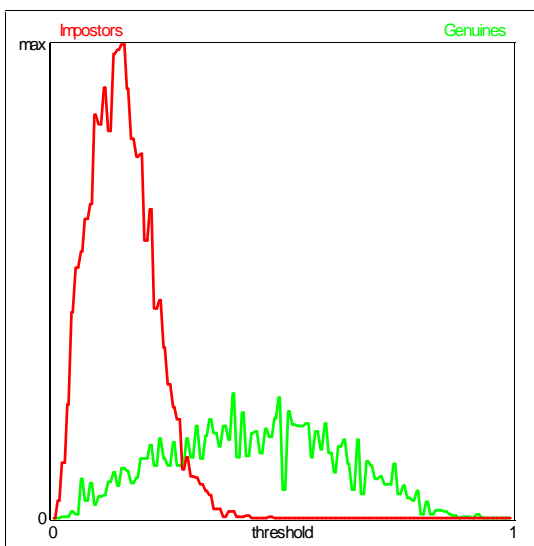

Score distributions

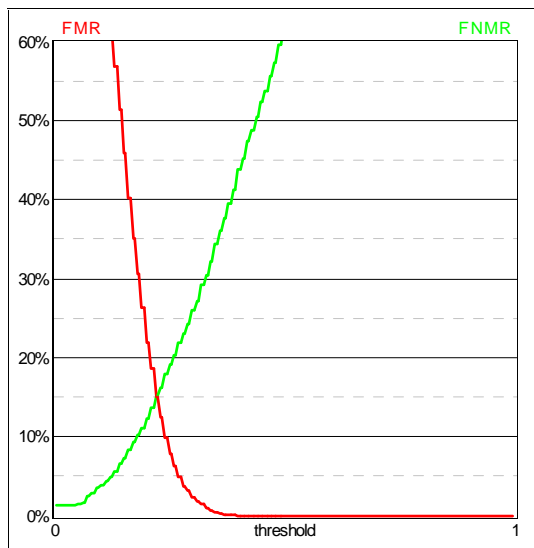

$\operatorname{FMR}(\mathrm{t})$ and $\operatorname{FNMR}(\mathrm{t})$

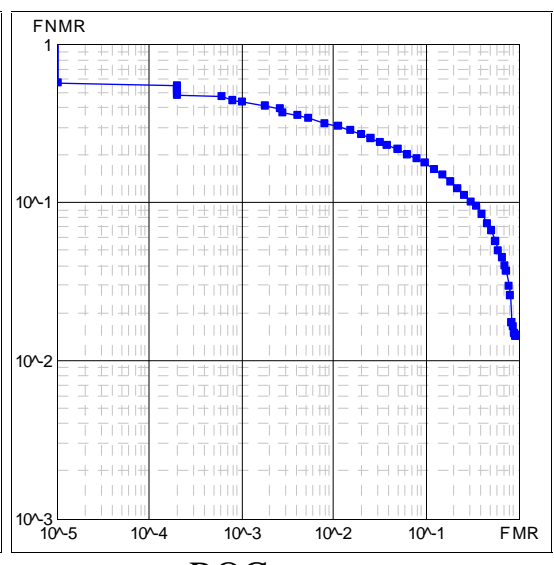

ROC curve 


\section{Algorithm Uinh on database $D B 3 \_A$}

Average enroll time: 1.28 seconds Average match time: 1.36 seconds

\begin{tabular}{|c|c|c|c|c|}
\hline REJ $_{\text {ENROLL }}$ & NGRA & NIRA & REJ $_{\text {NGRA }}$ & REJ $_{\text {NIRA }}$ \\
\hline $10.29 \%(\mathrm{~F}: 72 \mathrm{~T}: 0 \mathrm{C}: 0)$ & 2551 & 4581 & $9.21 \%(\mathrm{~F}: 235 \mathrm{~T}: 0 \mathrm{C}: 0)$ & $6.77 \%(\mathrm{~F}: 310 \mathrm{~T}: 0 \mathrm{C}: 0)$ \\
\hline EER & \multicolumn{2}{|c|}{ EER* } & ZeroFMR & ZeroFNMR \\
\hline $16.32 \%(16.13 \%-16.50 \%)$ & \multicolumn{2}{|c|}{$10.14 \%$} & $41.24 \%$ & $100.00 \%$ \\
\hline
\end{tabular}

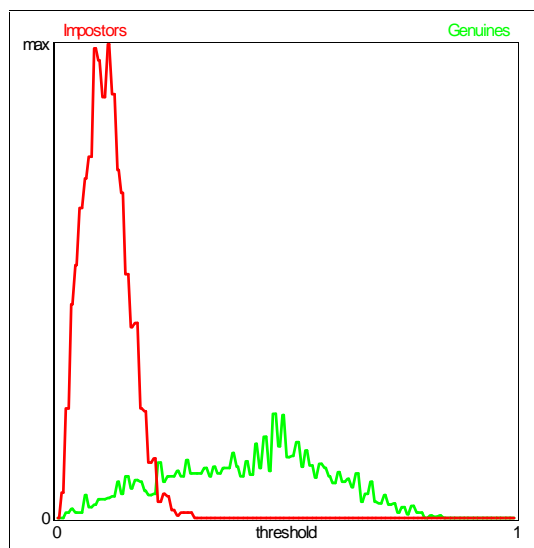

Score distributions

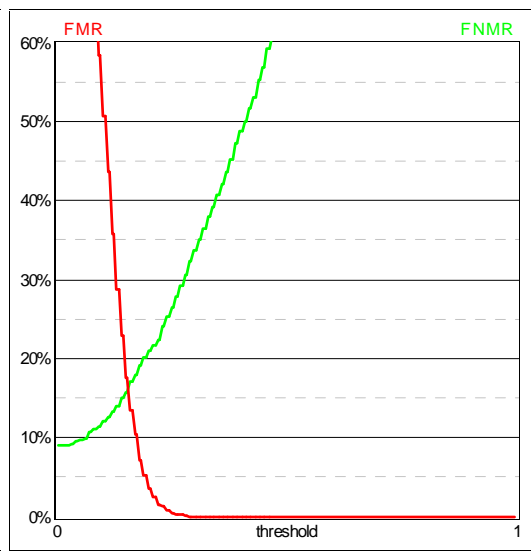

FMR(t) and FNMR(t)

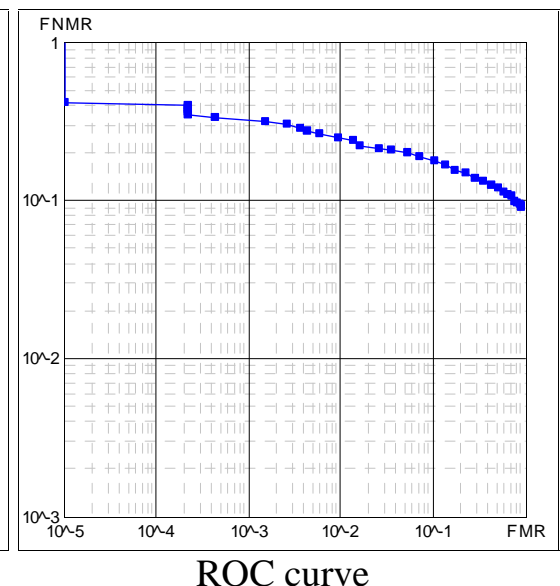

ROC curve

\section{Algorithm Uinh on database $D B 4 \_A$}

Average enroll time: 0.42 seconds

Average match time: 0.45 seconds

\begin{tabular}{|c|c|c|c|c|}
\hline REJ $_{\text {ENROLL }}$ & NGRA & NIRA & REJ $_{\text {NGRA }}$ & REJ $_{\text {NIRA }}$ \\
\hline $2.14 \%(\mathrm{~F}: 15 \mathrm{~T}: 0 \mathrm{C}: 0)$ & 2738 & 4839 & $2.81 \%(\mathrm{~F}: 77 \mathrm{~T}: 0 \mathrm{C}: 0)$ & $5.10 \%(\mathrm{~F}: 247 \mathrm{~T}: 0 \mathrm{C}: 0)$ \\
\hline \multicolumn{2}{|c|}{ EER $^{*}$} & ZeroFMR & ZeroFNMR \\
\hline EER & \multicolumn{3}{|c|}{$97.22 \%$} & $100.00 \%$ \\
\hline
\end{tabular}

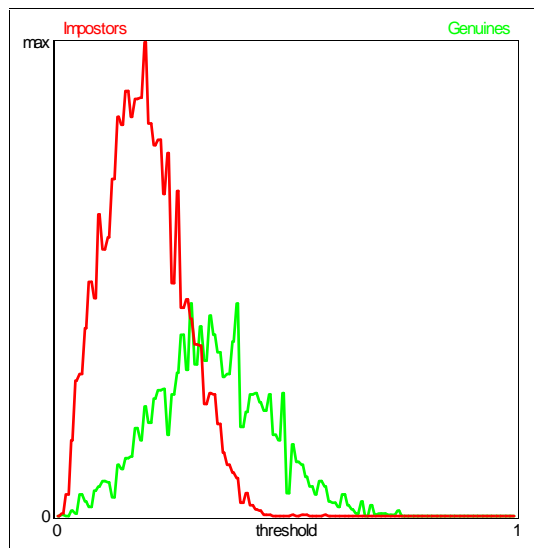

Score distributions

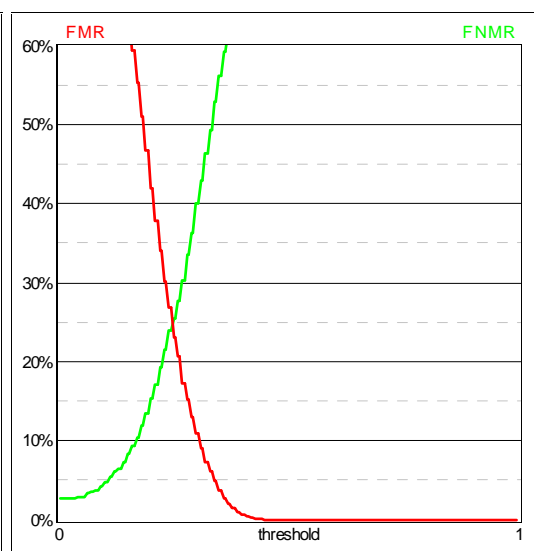

FMR(t) and $\operatorname{FNMR}(\mathrm{t})$

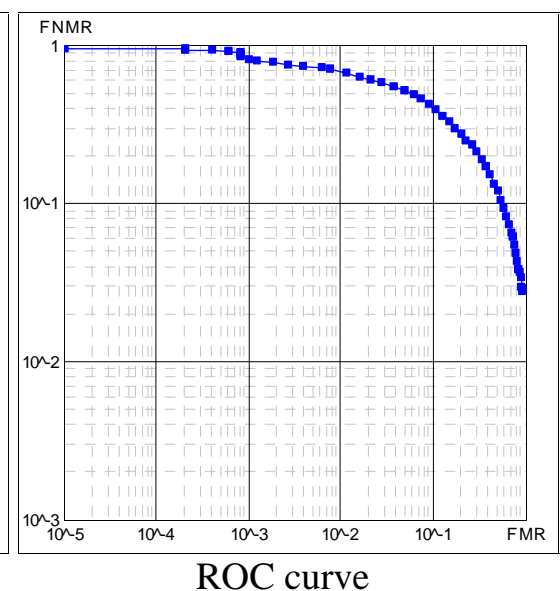




\section{Algorithm Utwe on database $D B 1 \_A$}

Average enroll time: 10.40 seconds

Average match time: 2.10 seconds

\begin{tabular}{|c|c|c|c|c|}
\hline REJ $_{\text {ENROLL }}$ & NGRA & NIRA & REJ $_{\text {NGRA }}$ & REJ $_{\text {NIRA }}$ \\
\hline $0.00 \%(\mathrm{~F}: 0 \mathrm{~T}: 0 \mathrm{C}: 0)$ & 2800 & 4950 & $0.00 \%(\mathrm{~F}: 0 \mathrm{~T}: 0 \mathrm{C}: 0)$ & $0.00 \%(\mathrm{~F}: 0 \mathrm{~T}: 0 \mathrm{C}: 0)$ \\
\hline
\end{tabular}

\begin{tabular}{|c|c|c|c|}
\hline EER & EER* & ZeroFMR & ZeroFNMR \\
\hline $7.98 \%(4.00 \%-11.96 \%)$ & $7.98 \%(4.00 \%-11.96 \%)$ & $44.00 \%$ & $100.00 \%$ \\
\hline
\end{tabular}

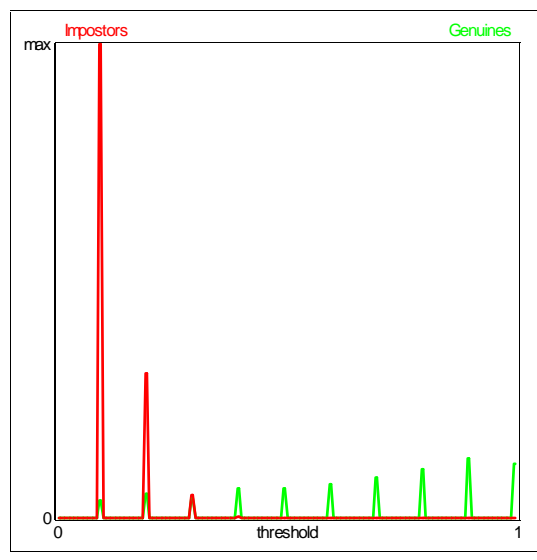

Score distributions

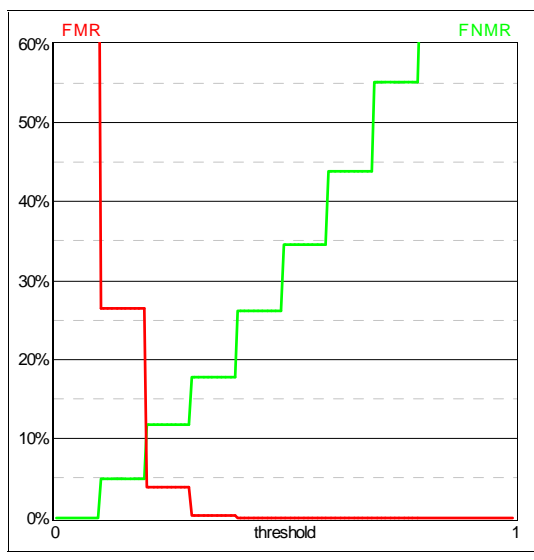

FMR (t) and FNMR(t)

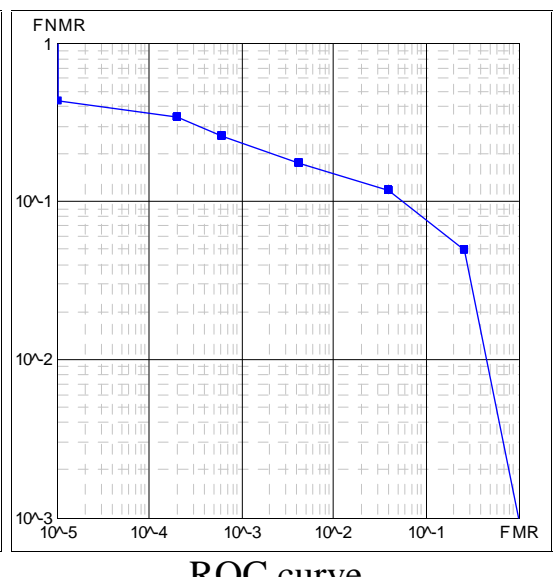

ROC curve

\section{Algorithm Utwe on database $D B 2 A$}

Average enroll time: 10.42 seconds

Average match time: 2.12 seconds

\begin{tabular}{|c|c|c|c|c|}
\hline REJ $_{\text {ENROLL }}$ & NGRA & NIRA & REJ $_{\text {NGRA }}$ & REJ $_{\text {NIRA }}$ \\
\hline $0.00 \%(\mathrm{~F}: 0 \mathrm{~T}: 0 \mathrm{C}: 0)$ & 2800 & 4950 & $0.00 \%(\mathrm{~F}: 0 \mathrm{~T}: 0 \mathrm{C}: 0)$ & $0.00 \%(\mathrm{~F}: 0 \mathrm{~T}: 0 \mathrm{C}: 0)$ \\
\hline
\end{tabular}

\begin{tabular}{|c|c|c|c|}
\hline EER & EER* & ZeroFMR & ZeroFNMR \\
\hline $10.65 \%(4.16 \%-17.14 \%)$ & $10.65 \%(4.16 \%-17.14 \%)$ & $46.57 \%$ & $100.00 \%$ \\
\hline
\end{tabular}

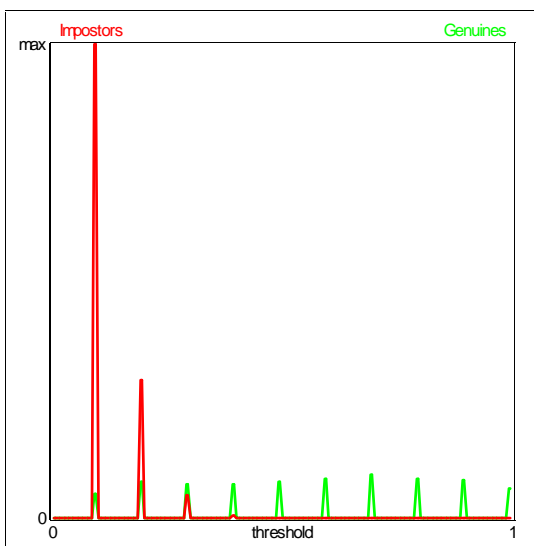

Score distributions

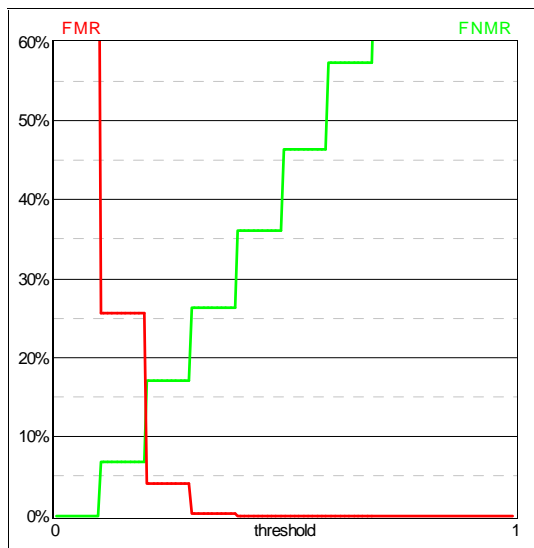

$\operatorname{FMR}(\mathrm{t})$ and $\mathrm{FNMR}(\mathrm{t})$

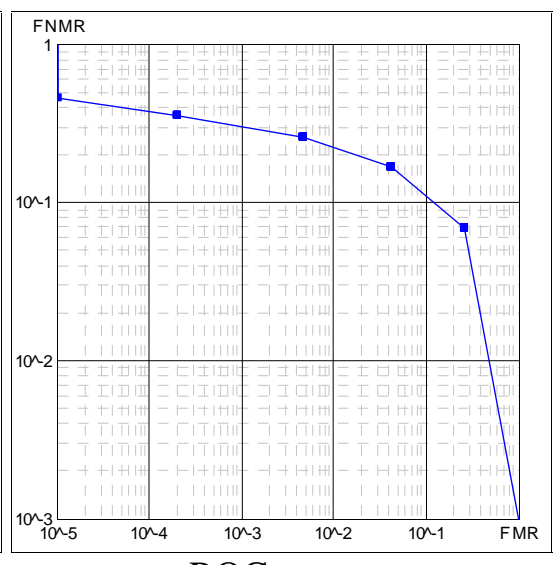

ROC curve 


\section{Algorithm Utwe on database $D B 3 \_A$}

Average enroll time: 10.44 seconds Average match time: 2.31 seconds

\begin{tabular}{|c|c|c|c|c|}
\hline REJ J & NGRA & NIRA & REJ $_{\text {NGRA }}$ & REJ $_{\text {NIRA }}$ \\
\hline $0.00 \%(\mathrm{~F}: 0 \mathrm{~T}: 0 \mathrm{C}: 0)$ & 2800 & 4950 & $0.00 \%$ (F:0 T:0 C:0) & $0.00 \%$ (F:0 T:0 C:0) \\
\hline EER & \multicolumn{2}{|c|}{ EER* } & ZeroFMR & ZeroFNMR \\
\hline $17.73 \%(4.06 \%-31.39 \%)$ & \multicolumn{2}{|c|}{$17.73 \%(4.06 \%-31.39 \%)$} & $68.82 \%$ & $100.00 \%$ \\
\hline
\end{tabular}

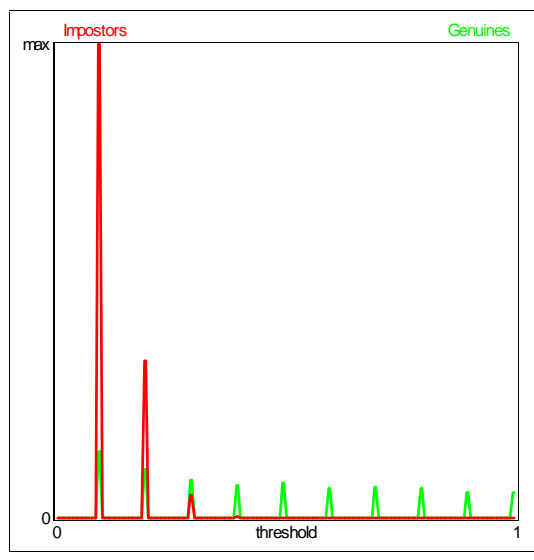

Score distributions

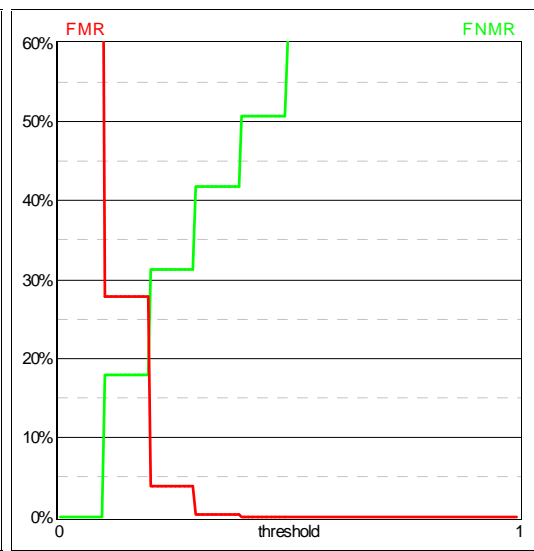

FMR(t) and FNMR(t)

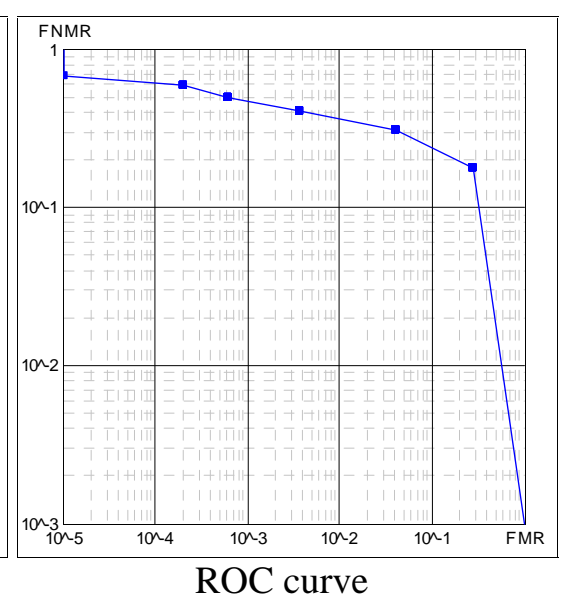

\section{Algorithm Utwe on database $D B 4 \_A$}

Average enroll time: 10.42 seconds Average match time: 4.17 seconds

\begin{tabular}{|c|c|c|c|c|}
\hline REJ $_{\text {ENROLL }}$ & NGRA & NIRA & REJ $_{\text {NGRA }}$ & $\mathbf{R E J}_{\text {NIRA }}$ \\
\hline $0.00 \%(\mathrm{~F}: 0 \mathrm{~T}: 0 \mathrm{C}: 0)$ & 2800 & 4950 & $0.00 \%(\mathrm{~F}: 0 \mathrm{~T}: 0 \mathrm{C}: 0)$ & $0.00 \%$ (F:0 T:0 C:0) \\
\hline EER & \multicolumn{2}{|c|}{ EER* } & ZeroFMR & ZeroFNMR \\
\hline $24.59 \%(4.79 \%-44.39 \%)$ & \multicolumn{2}{|c|}{$24.59 \%(4.79 \%-44.39 \%)$} & $94.29 \%$ & $100.00 \%$ \\
\hline
\end{tabular}

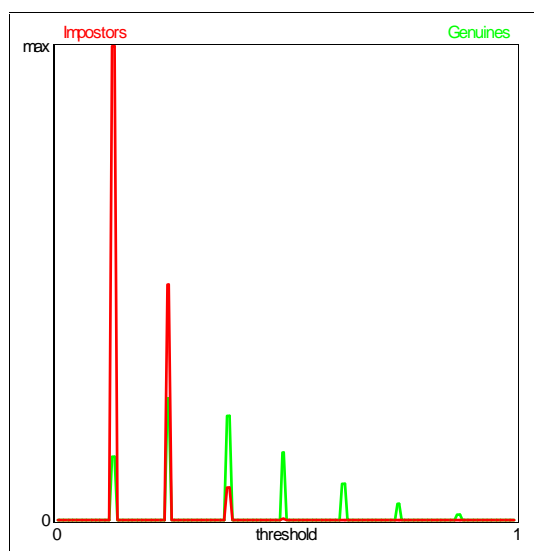

Score distributions

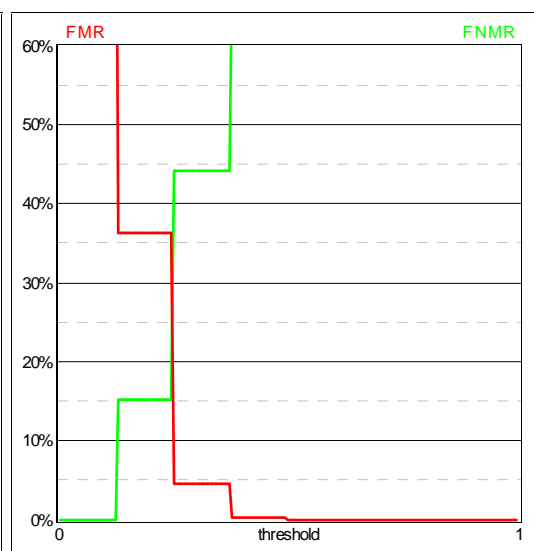

FMR(t) and $\operatorname{FNMR}(\mathrm{t})$

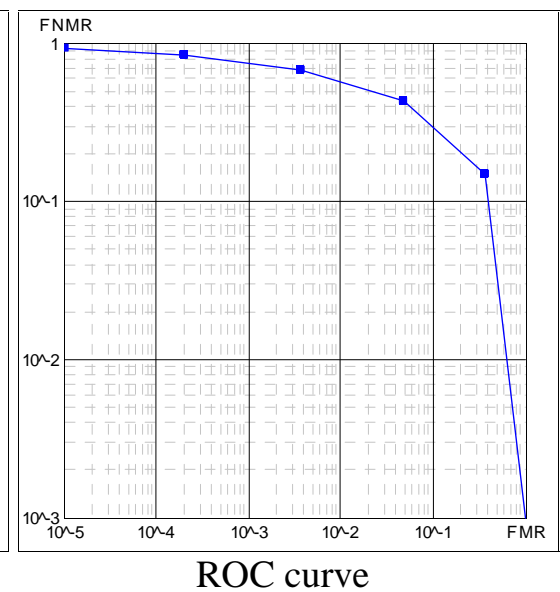

